\title{
TDP-43 extracted from FTLD patient brains displays distinct aggregate assemblies and neurotoxic effects reflecting disease progression rates
}

Florent Laferrière ${ }^{1 *}$, Zuzanna Maniecka ${ }^{1 *}$, Manuela Pérez-Berlanga ${ }^{1}$, Marian Hruska-Plochan ${ }^{1}$, Larissa Gilhespy $^{1}$, Eva-Maria Hock ${ }^{1}$, Ulrich Wagner ${ }^{2}$, Tariq Afroz ${ }^{1}$, Paul J. Boersema ${ }^{3}$, Gery Barmettler ${ }^{4}$, Sandrine C. Foti ${ }^{5,6}$, Yasmine T. Asi ${ }^{5,6}$, Adrian M. Isaacs ${ }^{6,7}$, Ashraf Al-Amoudi ${ }^{8}$, Amanda Lewis ${ }^{8}$, Henning Stahlberg ${ }^{8}$, John Ravits $^{9}$, Francesca De Giorgi ${ }^{10,11,12}$, François Ichas $^{10,11,12}$, Erwan Bezard $^{11,12}$, Paola Picotti ${ }^{4}$, Tammaryn Lashley ${ }^{5,6}$, Magdalini Polymenidou ${ }^{1}$

${ }^{1}$ Institute of Molecular Life Sciences, University of Zurich, Winterthurerstrasse 190, CH-8057 Zurich, Switzerland

${ }^{2}$ Department of Pathology and Molecular Pathology, University Hospital Zurich, CH-8091 Zurich, Switzerland

${ }^{3}$ Institute of Biochemistry, Department of Biology, ETH Zurich (ETHZ), CH-8093 Zurich, Switzerland

${ }^{4}$ Center for Microscopy and Image Analysis, University of Zurich, $\mathrm{CH}-8057$ Zurich, Switzerland

${ }^{5}$ Queen Square Brain Bank for Neurological diseases, Department of Movement Disorders, UCL Institute of Neurology, London, WC1N 1PJ, UK

${ }^{6}$ Department of Neurodegenerative Disease, UCL Institute of Neurology, Queen Square, London, WC1N 3BG, UK

${ }^{7}$ UK Dementia Research Institute at UCL, UCL Institute of Neurology, Queen Square, London, WC1N 3BG, UK

${ }^{8}$ Center for Cellular Imaging and NanoAnalytics (C-CINA), Biozentrum, University of Basel, $\mathrm{CH}-4058$ Basel, Switzerland

${ }^{9}$ Department of Neuroscience, University of California, San Diego, 9500 Gilman Drive, La Jolla, CA, 92093-0624, USA

${ }^{10}$ INSERM U1084, Laboratoire des Neurosciences Expérimentales et Cliniques, Université de Poitiers, 86073 Poitiers, France

${ }^{11}$ Université de Bordeaux, Institut des Maladies Neurodégénératives, UMR 5293, 33076 Bordeaux, France

${ }^{12}$ CNRS, Institut des Maladies Neurodégénératives, UMR 5293, F-33076, Bordeaux, France

*These authors contributed equally to this work

\section{Author for correspondence:}

Magdalini Polymenidou, PhD

Institute of Molecular Life Sciences, Y32-J06

University of Zurich

Winterthurerstrasse 190,

$\mathrm{CH}-8057$ Zurich

Office: +41-44-635-3106

Cell: +41-79-137-6856

email: magdalini.polymenidou@imls.uzh.ch

Keywords - ALS, FTD, FTLD, TDP-43, protein aggregation, neurotoxicity, prion-like seeding 


\begin{abstract}
Accumulation of abnormally phosphorylated TDP-43 (pTDP-43) is the main pathology in affected neurons of patients with amyotrophic lateral sclerosis (ALS) and frontotemporal lobar degeneration (FTLD). Morphological diversity and neuroanatomical distribution of pTDP-43 accumulations allowed classification of FTLD cases into at least four subtypes, which correlate with clinical presentations and genetic causes. To understand the molecular basis of this heterogeneity, we developed SarkoSpin, a new method for biochemical isolation of pathological TDP-43. Combining SarkoSpin with mass spectrometry, we revealed proteins beyond TDP-43, which become abnormally insoluble in a disease subtype-specific manner. We show that pTDP-43 extracted from brain forms stable assemblies of distinct densities and morphologies that correlate with disease subtypes. Importantly, biochemically extracted pTDP-43 assemblies displayed differential neurotoxicity and seeding that correlated with disease duration of FTLD patients. Our data are consistent with the notion that disease heterogeneity could originate from alternate pathological TDP-43 conformations, reminiscent of prion strains.
\end{abstract}




\section{Introduction}

Amyotrophic lateral sclerosis (ALS) and frontotemporal lobar degeneration (FTLD) are devastating neurodegenerative disorders sharing genetic, neuropathological and clinical features ${ }^{1}$. Both diseases are characterized by pathological, ubiquitinated protein inclusions in affected neurons, which in almost all ALS and $\sim 45 \%$ of FTLD patients contain the RNA/DNA-binding protein TDP- $43^{2,3}$. TDP-43 is a member of the heterogeneous nuclear ribonucleoprotein (hnRNP) family ${ }^{4}$ and plays essential roles in splicing regulation ${ }^{5,6}$ which are dependent on the formation of physiological homo-oligomers ${ }^{7,8,9}$. TDP43 is predominantly nuclear, but can shuttle to the cytoplasm ${ }^{10}$, where it incorporates into stress ${ }^{11}$ and transporting $\mathrm{RNA}^{12,13}$ granules via its low complexity domain (LCD), which mediates phase separation $^{14,15}$. FTLD- and ALS-associated TDP-43 was shown to undergo several abnormal posttranslational modifications including poly-ubiquitination, hyper-phosphorylation and proteolytic cleavage $^{2,3}$. The latter leads to the formation of highly aggregation-prone C-terminal fragments that are deposited in inclusions along with full-length TDP-43 ${ }^{16,17}$.

TDP-43 neuropathology manifests in diverse histological patterns, which correlate with clinical presentations $^{18}$, including behavioral variant frontotemporal dementia (bvFTD), semantic dementia (SD) and primary non-fluent aphasia $(P N F A)^{19}$. Based on morphology and neuroanatomical distribution of pathological TDP-43, FTLD-TDP can be classified into the following subtypes ${ }^{20,21}$. FTLD-TDP type A (FTLD-TDP-A) is characterized by neuronal cytoplasmic inclusions (NCI), dystrophic neurites (DN) and neuronal intranuclear inclusions (NII) in upper cortical layers and typically manifests with bvFTD, but no MND. Some FTLD-TDP-A patients carry GRN or C9ORF72 mutations, which are non-distinguishable from sporadic cases. FTLD-TDP type B cases (FTLD-TDP$\mathrm{B})$, which can also be linked to C9ORF72 mutations, show primarily granular $\mathrm{NCl}$ expanding across all cortical layers, and present with a combination of FTD and MND. Finally, FTLD-TDP type C (FTLDTDP-C) comprises long and thick $\mathrm{DN}$ and few $\mathrm{NCl}$ primarily in superficial cortical layers. These cases are sporadic and show SD without MND. Recently, other subtypes, which do not fully adhere to these pathological classification criteria were described ${ }^{20,21}$, suggesting that we are only beginning to identify 
the full spectrum of disease heterogeneity, whose origin remains unknown, but was proposed to correlate with specific molecular signatures ${ }^{22,23}$.

To address the molecular underpinnings of disease heterogeneity, we sought to analyze the biochemical characteristics of TDP-43 aggregates, which would require their physical separation from physiological TDP-43 and other proteins. We developed SarkoSpin, a novel, simple method for pathological TDP-43 extraction. Using SarkoSpin on brain cortical samples from $>80$ patients, we found that TDP-43 forms large assemblies of distinct densities, polyubiquitination levels and morphologies that correlate with specific neuropathological classifications. Coupling SarkoSpin with mass spectrometry, we identified a subset of insoluble proteins, beyond TDP-43, in each disease subtype. These proteins rarely co-aggregate with pTDP-43 and most likely represent a downstream effect of TDP-43 pathology. Importantly, we show that SarkoSpin-extracted pTDP-43 assemblies exhibit cytotoxicity and protein seeding with potency that reflects the disease duration of the respective subtype. Collectively, our data demonstrate that FTLD heterogeneity is reflected in the biochemical, neurotoxic and seeding properties of TDP-43. We propose that alternative TDP-43 pathological conformations may underlie the diversity of TDP-43 proteinopathies, reminiscent of prion strains $^{24,25}$.

\section{Results}

\section{Summary of patient cohort and characterization of FTLD-TDP-A and FTLD-TDP-C cases}

Brain cortical samples from over 80 patients, including control patients with no apparent CNS pathology or with non-TDP-43-linked neurodegeneration, as well as ALS and FTLD patients of different subtypes were obtained from three different centers worldwide (Supplementary Table 1). The morphology and neuroanatomical distribution of pathological TDP-43 accumulations of these cases (Supplementary Fig. 1a) adhered to the current classifications ${ }^{20,21}$. FTLD-TDP-C patients in our cohort showed significantly longer disease duration compared to all other subtypes (Supplementary Fig. 1b-c), in agreement with a recent report ${ }^{21}$. 


\section{SarkoSpin: a new method for the isolation of pathological TDP-43 from human brain}

Although pathological TDP-43 is resistant to detergents, such as sarkosyl| $\left.\right|^{26,27,22,28}$, there is currently no method that allows the physical separation of pathological TDP-43 from the protein pool in complex tissues. Instead, physiological TDP-43, as well as other proteins containing low complexity domains, typically co-purify with pathological TDP-43 in sarkosyl-insoluble fractions from patient brain tissue ${ }^{27,28}$ (Supplementary Fig. 2a-b). This contamination is likely due to the formation of large complexes with nucleic acids, including physiological nuclear TDP-43 oligomers ${ }^{7}$, which become partially resistant to detergent solubilization (Supplementary Fig. 2b).

To overcome this limitation and to purify pathological TDP-43 from its normal counterpart, we combined harsh solubilization with nuclease treatment and a single centrifugation step in a new method we termed SarkoSpin (Fig. 1a-b). In order to assess the efficiency of our technique, we analyzed motor and frontal cortex homogenates from control, ALS and FTLD-TDP patients (Supplementary Table 1). Total protein amounts partitioning in the SarkoSpin supernatant and pellet fractions were estimated by silver staining of SDS-PAGE-analyzed samples (Fig. 1c and Supplementary Fig. 2c) and by measuring protein concentrations. On average, $99.8 \%$ of the total protein mass resided in the supernatant with no significant difference among groups (Fig. 1d). 99.9\% of total TDP-43 was found in the supernatants of control samples (Fig. 1e-f and Supplementary Fig. 3), while in disease cases this was only $88 \%$, indicating that the rest of TDP-43 was present in the sarkosyl-insoluble pellet, corresponding to the pathological species. Indeed, the pellet fraction presented the characteristic signature for pathological TDP-43, namely poly-ubiquitination, C-terminal cleavage and hyperphosphorylation, which was detected by antibodies against total TDP-43 or phosphorylated TDP-43 (pS409/410) (Fig. 1b, e, f and Supplementary Fig. 3), and showed 64\% enrichment in disease pellets with no significant difference between disease types (Fig. 19-h). Proteins with low-complexity domains or RNA/DNA binding ability, such as other members of the hnRNP family, as well as other ALS/FTD-associated proteins (FUS, hnRNPA1, hnRNPH, SOD1) and 
normal proteins partitioning in complex macromolecular assemblies (actin, histones), were undetectable in SarkoSpin pellets (Fig. 1i-j and Supplementary Fig. 3).

\section{SarkoSpin captures native pathological TDP-43 assemblies}

To further characterize the properties of these assemblies, we next performed native-PAGE of SarkoSpin samples. Supernatants of all cases including controls showed a wide smear, ranging from the stacking gel (>10 MDa) to the size of monomeric TDP-43, where a strong band was observed (Supplementary Fig. 4a), suggesting that TDP-43 occurs in different oligomeric states in agreement with our recent report ${ }^{7}$. Notably, total protein concentrations were equalized before SarkoSpin, explaining the lower signal observed in controls, which lack TDP-43 aggregates. Strikingly, a strong TDP-43 signal, also detectable with a pTDP-43-specific antibody was observed in the stacking gel in patient, but not control, samples (Supplementary Fig. 4b), corresponding to the pathological species. Importantly, in contrast to control samples, SarkoSpin-pelleted patient samples immunoblotted with total TDP-43- or pTDP-43-specific antibodies revealed solely the presence of high molecular weight assemblies retained in the stacking gels (Supplementary Fig. 4c-d), further confirming the efficient separation of pathological from physiological assemblies. If exclusively composed of TDP-43, such high ordered aggregates would consist of at least 200 monomers, which may represent the minimal unit comprising the large inclusions found in patient-affected neurons. While no differences were observed among the various disease types, we cannot exclude that subtype-specific differences occur above the resolved sizes, undetectable by the resolution of this assay.

\section{Insoluble proteome and levels of pathological TDP-43 ubiquitination distinguishes disease subtypes}

To explore which other proteins, beyond TDP-43, become insoluble in patient samples, we analyzed SarkoSpin preparations from brain homogenates of eleven controls and twenty-seven patients by liquid chromatography-mass spectrometry (LC-MS). After denaturation and normalization of protein 
concentrations, SarkoSpin pellets were subjected to enzymatic digestion. Tandem mass spectra acquired by high-resolution mass spectrometers were searched against a human protein database. Spectral counting was used for quantifying differences in protein abundance between samples. The most significantly enriched protein $\left(p=10^{-3}\right)$ and most consistently correlating $(r=0.65)$ with FTLDTDP, but not control or FTLD-Tau SarkoSpin pellets was TDP-43 (Supplementary Fig. 5). All remaining proteins had weaker correlation values $(r<0.5)$ and were less consistently enriched in the patient group, illustrating that indeed TDP-43 is the main component of insoluble protein aggregates ${ }^{3,2}$ and further validating SarkoSpin as a reliable method for their isolation.

Importantly, when we compared the insoluble proteins enriched in each disease group separately (Supplementary Fig. 6-9), we found that a distinct set of proteins characterized each subtype (Fig. 2a). This suggests that while TDP-43 is the only protein consistently aggregating in disease, a specific subclass of proteins accompany TDP-43 within the insoluble fraction of each disease group. To confirm these findings, we first tested several commercially available antibodies for sensitivity and specificity to proteins predicted to correlate with pTDP-43 levels in specific disease subtypes (Fig. 2a). Based on their performance, we selected one protein per disease group, namely: profilin 1 (PFN1), enriched in ALS cases and reported to be mutated in rare familial ALS cases ${ }^{29,30}, \mathrm{~N}$ acylsphingosine amidohydrolase (ASAH1), enriched in FTLD-TDP-A cases and mutated in some cases of spinal muscular atrophy ${ }^{31}$ and Thioredoxin-like protein 1 (TXNL1), enriched in FTLD-TDP-C in our dataset. We then tested their presence in SarkoSpin fractions of individual patients by immunoblot and found that, while their levels on SarkoSpin supernatants were comparable (Supplementary Fig. 10a-b), they were enriched in the pellets of samples from TDP-43 proteinopathies (Fig. 2b). Notably, this enrichment best correlated with pTDP-43 levels in the respective disease subtype (Fig. 2c), in agreement with our mass spectrometry results. Despite the large variability in pTDP-43 levels of individual patient samples, we confirmed the validity of these correlations, by the almost perfect correlation for all groups with total TDP-43 in these pellets (Supplementary Fig. 10c-d). 
A similar approach was used to correlate ubiquitin and pTDP-43 levels in SarkoSpin pellets, which revealed an interesting and unexpected observation. Ubiquitin blots of SarkoSpin pellets and supernatants showed a completely different pattern, with ubiquitin-positive smears characteristic for pathological polyubiquitination present only in the pellets (Fig. 2d), confirming that SarkoSpin specifically concentrated misfolded proteins that escape proteasome degradation. Surprisingly, ubiquitin levels of pTDP-43 extracted from FTLD-TDP-C patients were consistently lower than those derived from FTLD-TDP-A and ALS patients (Fig. 2d-e). Comparison of co-immunofluorescence analysis of pTDP-43 and ubiquitin on patient brain sections confirmed the differential levels of pTDP43 polyubiquitination (Fig. 2f-g and Supplementary Fig. 10e).

With the exception of a few identified proteins such as LAMA1 and HepaCAM, which belong to the extracellular matrix - and most probably associate with TDP-43 after tissue lysis - all other proteins found in patient SarkoSpin pellets are localized intracellularly. To investigate if the increased insolubility of ASAH1 and TXNL1 resulted from direct co-aggregation with pTDP-43, or if these proteins become insoluble as a downstream event of TDP-43 aggregation, similar to findings in other proteinopathies ${ }^{32}$, we performed co-immunofluorescence analysis with pTDP-43 on brain sections. ASAH1 and TXNL1 both demonstrated cytoplasmic staining, which was increased in levels and displayed a punctate pattern in both patient subtypes (Supplementary Fig. 11). This pattern appeared largely independent from pTDP-43 inclusions, with rare co-localization observable in both disease subtypes. This suggested that increased insolubility of these proteins in disease does not result from direct interaction and co-aggregation with pTDP-43 and that SarkoSpin likely dissociated other proteins previously shown to co-aggregate with pTDP-43 on patient tissues ${ }^{33}$, thereby releasing the pTDP-43 "core" of these pathological assemblies.

Importantly, one of the proteins enriched in FTLD-TDP-A in our mass spectroscopy analysis was the F-Box Protein 2 (FBXO2), which during our initial screen by conventional immunohistochemistry stained neuronal cytoplasm in control and FTLD-TDP-C brain sections, while in the FTLD-TDP-A subtype a characteristic astrocytic-like pattern was observed (Supplementary Fig. 12a). To further 
explore this finding, we performed co-immunofluorescence analysis of FBXO2 and the astrocytic marker glial fibrillary acidic protein (GFAP). Excitingly, while in both disease subtypes astrogliosis was evident by increased GFAP staining, FBXO2 marked FTLD-TDP type A, but not type C astrocytes (Fig. 2h and Supplementary Fig. 12b). These data indicate a previously unrecognized distinct astrocytic reaction characterizing FTLD-TDP-A patients, illustrating divergent pathogenic mechanisms within these two disease subtypes.

\section{Diverse size distributions of TDP-43 aggregates isolated from different types of TDP-43 proteinopathies}

We next assessed the sedimentation profiles of TDP-43 aggregates to uncover specific size or shape differences among disease subtypes. Brain cortex homogenates of eight controls and twenty-seven patients were SarkoSpin-solubilized and layered on top of linearized iodixanol gradients before ultracentrifugation (Fig. 3a). Gradients were fractionated in 16 fractions of equal volume and TDP-43 or pTDP-43 distribution was assessed by immunoblotting. In both control and disease samples monomeric, as well as oligomeric TDP-43, were retained in the top five fractions, peaking in the first three (Fig. 3b-d and Supplementary Fig. 13-14a-b). In addition, populations of larger sizes, carrying the specific post-translational modifications of pathological TDP-43 were identified specifically in disease cases. In ALS samples, sedimentation profiles revealed one population of aggregated and phosphorylated TDP-43 in fractions 8 to 13 and peaking between 10-11. FTLD-TDP-A sedimentations revealed a wide diversity of profiles, with TDP-43 aggregates sedimenting in fractions 5 to 14 , and two apparent populations peaking in fractions 6-7 and in fraction 11. The presence of a second subpopulation could indicate a difference in size or shape of the aggregates. Importantly, neuropathological type rather than C9ORF72 mutation better correlated with the size distribution of TDP-43 aggregates, as they followed the pattern observed for the respective disease type (peaks in fractions 6-7 and 11 for type A and fractions 10-12 for type B) and clearly separated in different groups. FTLD-TDP-C sedimentation profiles also revealed one broad population of TDP-43 
aggregates (Fig. 3b-c and Supplementary Fig. 13-14a-b). However, in contrast to the other types, these assemblies were sedimenting further, in fractions 10-16, which may reflect their bigger size and/or higher intrinsic density. Importantly, hnRNPA1, FUS and SOD1 remained in the first three fractions in all disease types and controls (Supplementary Fig. 14c), indicating that solubility of these proteins is not significantly altered in TDP-43 proteinopathies.

\section{Distinct density profiles of TDP-43 aggregates isolated from different types of TDP-43 proteinopathies}

To determine whether the observed differences in velocity sedimentation were due to distinct aggregate size, shape or density, we sought out to measure the intrinsic density of TDP-43 aggregates. To this end, we submitted the same patient samples to SarkoSpin solubilization, followed by ultracentrifugation in an iodixanol discontinuous gradient for 17 hours to reach the isopycnic equilibrium ${ }^{24}$ (Fig. 4a). Gradients were then fractionated and TDP-43 and pTDP-43 density profiles were determined as described above (Fig. 4b-d and Supplementary Fig. 15-16a-b).

In all samples, the majority of normal TDP-43 was present in one peak spanning fractions 8 to 12 , i.e. at an average density of $1.22 \mathrm{~g} / \mathrm{mL}$. Remarkably, all density gradients from ALS, FTLD-TDP-A (Fig. 4b-c) and FTLD-TDP-B cases (Supplementary Fig. 15a) showed an additional population of TDP43 , present in one sharp peak in fraction 7 , corresponding to density of $1.16 \mathrm{~g} / \mathrm{mL}$. This consistent population demonstrated all known TDP-43 pathological features namely ubiquitination, C-terminal cleavage, and phosphorylation (Supplementary Fig. 16a-b). Surprisingly, density profiles of all nine FTLD-TDP-C samples were significantly different from all other types of TDP-43 proteinopathies tested with a peak of pTDP-43 intermingled with the normal TDP-43 peak in fractions 8 to 10 , i.e. 1.20 g/mL (Fig. 4b-c, Supplementary Fig. 15b-16b).

We next performed native-PAGE immunoblots on density floatation gradient fractions. Two major populations were distinguishable in all cases, including controls: one in fractions 8-12 under $66 \mathrm{kDa}$, and one in fractions 5-7 smearing from $66 \mathrm{kDa}$ to $10 \mathrm{MDa}$. The latter represented TDP-43 oligomers ${ }^{7}$, 
which were more buoyant than monomeric species and comprised an estimated $25-35 \%$ of total TDP43 in controls. Importantly, in agreement with Fig. 4, the high molecular weight pTDP-43 aggregates were present in fractions 7 and 8-10 for FTLD-TDP-A and FTLD-TDP-C, respectively (Supplementary Fig. 16c). Collectively, these results strikingly show that pathological TDP-43 aggregates extracted from FTLD-TDP-C were ordered in a different fashion than those found in other FTLD-TDP subtypes or ALS.

\section{Alternate morphologies of TDP-43 aggregates isolated from different disease subtypes}

In order to visualize TDP-43 aggregates, anti-TDP-43 immunogold labeling followed by transmission electron microscopy (TEM) was performed on SarkoSpin pellets. To avoid drying artifacts samples were embedded in methylcellulose. No immunoreactivity or large protein assemblies were detected in control samples. In contrast, FTLD-TDP-A and FTLD-TDP-C SarkoSpin pellets contained numerous protein structures of various shapes and sizes, ranging from 50 to $150 \mathrm{~nm}$, and decorated by gold bead-labeled anti-TDP-43 antibody (Fig. 5a, red arrows). Interestingly, although the same monoclonal antibody recognizing a short epitope spanning amino acids 203-209 did not show consistent differences between the two disease groups under denaturing conditions, immunogold-EM revealed differential immunoreactivity, with strongly immuno-positive FTLD-TDP-A assemblies and only sparsely labeled FTLD-TDP-C aggregates. This lower antibody detection points to distinct epitope accessibility, and thus different packaging of pathological TDP-43 type A versus type $C$ aggregates. It is conceivable that the antibody epitope, which lies within the second RNA recognition motif (RRM2), is buried inside the core of FTLD-TDP-C aggregates. These results corroborate the higher density of type $\mathrm{C}$ aggregates and their discriminating three-dimensional shapes compared to type $\mathrm{A}$.

Notably, we did not detect any long amyloid fibrils in any disease case, in agreement with the apparent lack of amyloidophilic staining in the majority of ALS/FTLD patient brains ${ }^{34,35}$. Nevertheless, careful observation of the immunogold-EM images suggested the presence of short proteinaceous filaments, which were 5-8 $\mathrm{nm}$ thick and $30-50 \mathrm{~nm}$ long and were frequently decorated by the anti- 
TDP-43 antibody in FTLD-TDP-A (Fig. 5a, orange arrows). Similar structures were detected in FTLDTDP-C samples, albeit mostly independently from antibody labeling (Fig. $\mathbf{5 b}$, orange arrows). These structures potentially originated from filamentous material in FTLD brains as previously reported ${ }^{36}$, which would be in agreement with a recent study showing high-resolution structure of a short TDP-43 LCD-derived peptide ${ }^{37}$.

As the use of methylcellulose lowers image resolution, immunolabelled SarkoSpin pellets prepared without this preservation step were also analyzed by TEM. This allowed us to obtain higher resolution images of type A TDP-43 aggregates. These showed heterogenous spheroid or elongated and coiled protein assemblies (Fig. 5c). Due to lower immunoreactivity, such structures could not be identified in FTLD-TDP-C samples. Importantly, both methods for immunogold-labeled sample preparation showed no unspecific binding of the gold-conjugated secondary antibody (Supplementary Fig. 17a). Lipids, sometimes appearing adjacent to TDP-43 aggregates (Fig. 5a, yellow arrows), could be removed by a slight adaptation of SarkoSpin method with centrifugation over a sucrose cushion (Supplementary Fig. 17b).

To obtain an independent, albeit indirect, measure of the different conformational features of TDP-43 aggregates, we used partial proteolysis. The latter has extensively been used as a surrogate for probing different pathological conformations of the prion protein, since the proteolytic fragment size is directly related to site accessibility to proteases ${ }^{25,38}$. In contrast to the prion protein, TDP-43 is largely proteinase K-sensitive (Supplementary Fig. 17c), but has previously been reported to be partially resistant to chymotrypsin and trypsin ${ }^{22,23}$. Here, we systematically analyzed the proteolytic profile of TDP-43 species extracted from different disease subtypes (Fig. 5d). Indeed, chymotrypsin and trypsin-treated SarkoSpin pellets showed specific and consistent proteolytic profiles in each disease subtype, suggesting that aggregate arrangement and, by extension, the borders of the proteaseresistant cores are distinctive. Additionally, probing with antibodies raised against defined TDP-43 domains revealed resistance of the most C-terminal fragment within the low-complexity domain, suggesting that the cleavage site is hidden within the aggregate core. Interestingly, these were found 
significantly more resistant to proteolysis by trypsin in FTLD-TDP-C compared to FTLD-TDP-A (Fig. 5e).

Lastly, we explored this proteolytic resistance of TDP-43 species to visualize the resistant cores of the aggregates, which we identified using immunogold labeling with an antibody against the TDP-43 phosphorylation sites at amino acids $409 / 410$ (Fig. 5f). This treatment resulted in protein structures with high anti-pTDP-43 immunopositivity for both FTLD-TDP-A and FTLD-TDP-C, further confirming that the lack of anti-RRM2 immunogold labeling in FTLD-TDP-C samples (Fig. 5a) was due to epitope accessibility within TDP-43 assemblies.

\section{Differential cyto- and neurotoxicity of FTLD-TDP type A and type $C$ pathological assemblies}

In order to determine whether these distinct TDP-43 conformations were associated with different toxic properties, we tested their effect on the viability of cell lines and primary neurons. To this end, we used the Flp-In T-Rex technology to generate a stable cell line with inducible expression of Cterminally tagged TDP-43, to obtain a homogeneous population of cells expressing traceable TDP-43 at similar levels (Supplementary Fig. 18a-c). We used the nine amino acid-long HA tag, which we placed at the very end of the structurally disordered region to minimize any interference with protein structure, which might hinder protein seeding. We first used our TDP-43-HA-expressing cells to optimize internalization of SarkoSpin-extracted GFP-labeled pTDP-43 aggregates produced in cells, demonstrating that our extraction method can also be applied to cellular models of disease (Supplementary Fig. 18d-f).

To test if SarkoSpin specifically concentrated toxic TDP-43 seeds, we subsequently introduced pellets from control and FTLD patients into mitotically arrested cells, since continuous proliferation and high metabolic activity of these cells could mask the toxicity of exogenous aggregates. We reasoned that if toxicity of pTDP-43 assemblies depended on seeding, the effect would be more profound upon increased expression of the substrate, i.e. TDP-43-HA. We therefore calculated the viability ratio of cells with or without TDP-43-HA expression and found that upon a 3-fold increase in TDP-43 protein 
levels (Supplementary Fig. 18g), cell viability was significantly decreased after inoculation with FTLD-TDP-A extracts in comparison to inoculation with control or FTLD-TDP-C aggregates (Fig. 6ab).

To directly test whether the observed toxicity is dependent on aggregation of endogenous TDP-43, we subsequently performed SarkoSpin on mitotically arrested TDP-43-HA-expressing cells inoculated with SarkoSpin pellet pools from control and FTLD patients. Immunoblot with anti-HA antibody against the endogenously expressed TDP-43-HA allowed for the unambiguous distinction of newly induced aggregates from exogenous patient-derived seeds. While levels of TDP-43-HA were equal in all conditions (Supplementary Fig. 18g), cells inoculated with FTLD-TDP-A pellets presented increased TDP-43-HA aggregation, compared to cells treated with control or FTLD-TDP-C seeds (Fig. 6c). Collectively, these data indicated that pTDP-43 assemblies extracted from FTLD-TDP-A, but not control or FTLD-TDP-C patients were cytotoxic and induced endogenous TDP-43 aggregation, potentially via protein seeding.

To confirm the increased potency of FTLD-TDP-A aggregates in a disease-relevant system, we quantified their effect on neurite length of primary cortical neurons, prepared from mouse embryos using high content screening ${ }^{39}$. Neurite length was previously found to be decreased by treatment with artificial oligomers of $\alpha$-synuclein, which are capable of entering cells and acting as seeds of an autonucleation process ${ }^{40}$. Neuronal cultures were exposed to four different doses of SarkoSpin pellet pools from control, FTLD-TDP-A or FTLD-TDP-C patients (Fig. 6d). TDP-43 and pTDP-43 were absent from control SarkoSpin pellets as expected, while their quantity in FTLD-TDP-C inocula was approximately half of FTLD-TDP-A for all doses (Supplementary Fig. 18i). Importantly, pTDP-43 levels were equivalent between FTLD-TDP-A at dose of $0.3 \mu \mathrm{g}$ and FTLD-TDP-C at $1 \mu \mathrm{g}$ total protein, which allowed the direct comparison of the two types of pathological pTDP-43 assemblies. The effect of exogenous pTDP-43 SarkoSpin extracts on neurite growth was then monitored by automated live cell imaging with combined phase-contrast and epifluorescence imaging over 10 days (Fig. 6e-f). In agreement with our observations in immortalized cells, TDP-43 aggregates extracted from FTLD- 
TDP-A drastically reduced neurite length (Fig. 6h), which reached a plateau immediately after inoculation (after 5 days in vitro (DIV)), while control or FTLD-TDP-C SarkoSpin pellets had no effect. At 10 DIV, all primary neuronal cultures were submitted to calcein fluorescence imaging allowing cell body counting and measuring neurite length (Fig. 6g-h). As seen in phase contrast, calcein staining of live cells decreased drastically and proportionally to the dose of FTLD-TDP-A-extracted aggregates, while control and FTLD-TDP-C SarkoSpin pellet inoculations had no effect. This specific neurotoxic effect of FTLD-TDP-A aggregates was measured both by the decrease of cell body number (Fig. $\mathbf{6 g}$ ) and neurite length (Fig. $\mathbf{6 h}$ ) in a dose-dependent manner.

Collectively, our data indicate that pathological TDP-43 adopts stable configurations with specific biophysical properties, which allow its physical separation from its physiological counterpart. Notably, the biochemical properties of pathological TDP-43 and a subset of insoluble proteins can discriminate between specific neuropathological subtypes, indicating that they may underlie specific disease characteristics. Most importantly, we show that pTDP-43 aggregates extracted by our new purification method confer cyto-/neurotoxicity accompanied by induced aggregation of endogenous TDP-43. Remarkably, the most toxic aggregates are those found in the brains of FTLD-TDP-A patients, who show significantly shorter disease duration (Supplementary Fig. 1c), suggesting a link between the properties of TDP-43 aggregates and clinical presentation.

\section{Discussion}

TDP-43 pathology characterizes most instances of ALS and FTLD and its spatiotemporal manifestation coincides with neurodegeneration, supporting its central pathogenic role in these diseases $^{41}$. Despite this unifying TDP-43 pathology, ALS and FTLD patients display large heterogeneity in terms of clinical presentation, progression and neuropathological findings, a phenomenon that remains mechanistically unexplained. Recent discoveries indicate that several protein aggregation diseases follow a prion-like paradigm ${ }^{42}$ through amplification of pathological conformers. These findings provide a plausible explanation of the origin of disease heterogeneity. 
Indeed, differential quaternary structures of the prion protein are responsible for different subtypes of infectious prion diseases and are associated with distinct neuropathological profiles and clinical presentations $^{38}$. The notion of prion strains was recently expanded to other protein aggregation diseases with compelling data for tau ${ }^{43}, \alpha$-synuclein ${ }^{44}$ and $A \beta^{45}$.

We show that human patient-derived TDP-43 adopts stable protein assemblies with specific biophysical properties. We exploited these features to develop SarkoSpin, a reliable method for the isolation of TDP-43 aggregates and their separation from physiological complexes. All pathological features described thus far for TDP-43 (namely insolubility, hyperphosphorylation, poly-ubiquitination, and proteolytic cleavage fragmentation) were concentrated in the SarkoSpin purified fraction. However, we cannot formally exclude that soluble pathogenic TDP-43 assemblies with so far undescribed pathological features escape our method and remain in the supernatant. Nevertheless, we foresee that SarkoSpin will become a valuable tool for any application requiring faithful, diseaseassociated TDP-43 aggregates. An unbiased proteomic approach of isolated pathological assemblies revealed that TDP-43 was the most significantly enriched protein and the only one found insoluble in all disease cases, indicating that we isolated the pTDP-43 core of pathological assemblies. Comparison of other proteins that become insoluble in ALS and FTLD human brains revealed specific subclasses of proteins characterizing each disease subtype. These proteins did not co-localize with pTDP-43 in patient brains and sometimes even occurred in different cell types - i.e. FBXO2 in astrocytes - suggesting that they most likely represent a downstream molecular signature of the divergent pathogenic mechanisms characterizing these disease subtypes.

Combining SarkoSpin with a number of biochemical, molecular and imaging methodologies, we provide evidence that TDP-43 extracted from different disease subtypes exhibits distinct biochemical and morphological features. In particular, the intrinsic density of the isolated particles is remarkably consistent within each disease subtype and a distinguishing feature of pathological TDP-43 in different FTLD types. Our results indicate that FTLD-TDP-C, an exclusively sporadic type of dementia with significantly longer disease duration than other types ${ }^{21}$, is associated with very dense TDP-43 
aggregates with lower levels of polyubiquitination and a highly protease resistant C-terminal core, compared to TDP-43 assemblies extracted from FTLD-TDP-A or ALS. While no long fibrillary structures were detected within TDP-43 aggregates of either disease subtype, short proteinaceous filaments were present in both, albeit with differential accessibility to specific antibodies, indicative of alternate aggregate packaging. Surprisingly, the neuropathological type, rather than genetic mutations, better predicted the features of TDP-43 aggregates, indicating that the same genetic trigger may give rise to variable pathological assemblies, perhaps determined by other, still unknown factors.

Most importantly, the biochemical and morphological differences between FTLD-TDP-A and FTLDTDP-C pTDP-43 assemblies were associated with differential seeding and neurotoxic potential. Indeed, FTLD-TDP-A aggregates extracted from patients with fast progressing disease caused toxicity and induced endogenous TDP-43 aggregation in cellular models, while FTLD-TDP-C aggregates from slow progressing disease seemed inert under the same conditions and equivalent pTDP-43 doses. Moving forward and following the paradigm now established for several other protein aggregates ${ }^{43,44,45}$, it would be exciting to find out whether these patient-derived TDP-43 pathological conformations can faithfully propagate in vivo, reproducing their distinctive features and disease phenotypes in animal models. Additional work is necessary to establish a direct link between TDP-43 pathological characteristics and disease subtypes. However, our work is compatible with the idea that disease heterogeneity could originate from alternate pathological TDP-43 conformations, as observed in the case of prion strains, and opens new avenues for creating cellular and animal models.

\section{Acknowledgements}

We are grateful to all the patients and their families for donating tissues for scientific research; this work would not have been possible without their generosity and foresightedness. We thank Shahram Saberi and Maria J Rodriguez from UCSD for preparing and shipping autopsy material from San Diego to Zurich; Andres Käch and the Center for Microscopy and Image Analysis of the University of 
Zurich for help and technical support in all EM experiments; Henning Leske, Karl Frontzek, Elisabeth Rushing, Adriano Aguzzi (Institute of Neuropathology, University Hospital of Zurich) for helpful advice and discussions; Julia Luedke and Julien Weber for technical help, Sonu Sahadevan M.K. and Aurelie Zbinden for critical input on the manuscript. We thank Dr. Gstaiger (Institute of Molecular Systems Biology, ETH Zurich, Switzerland) for kindly sharing the Flp-In T-REx HEK293 cell line and the pOG44 plasmid. This work was supported by a Swiss National Science Foundation Professorship (PP00P3_144862) and a Human Frontier Science Program Career Development Award (CDA00058/2012) to M.P. and a UCL/ZNZ Neuroscience Collaboration Grant to M.P and A.M.I funded through UCL's Wellcome Trust Institutional Strategic Support Fund 'Investing in Excellent Researchers' (105604/Z/14/Z). F.L. and M.H.P. are both recipients of the Milton-Safenowitz fellowship from the ALS Association (15-IIP-208 and 16-PDF-247, respectively). F.L. received a Postdoc Award and Z. M. a Candoc Award (Forschungskredit) from the University of Zurich. T.L. is funded by an Alzheimer's Research UK senior fellowship. Y.T.A. was supported by the Leonard Wolfson Centre for experimental neurology. Al is the recipient of an ERC consolidator grant (648716 - C9ND). The Queen Square Brain Bank is supported by the Reta Lila Weston Institute for Neurological Studies and the Progressive Supranuclear Palsy (Europe) Association.

\section{Author contributions}

F.L. developed and performed the biochemical experiments with the help of Z.M. and M.P. conceived and directed the study. M.P-B. generated and characterized the stable cell line with inducible TDP-43HA, in which she performed cell toxicity experiments. M.H-P., L.G., E-M.H., and T.A. helped with performing gradients and developing the SarkoSpin method. P.B. and P.P. performed mass spectrometry, which was bioinformatically analysed by U.W.. G.B. performed electron microscopy experiments. A.A-A., A.L. and H.S. gave critical input on the structural analysis of extracted aggregates by electron microscopy and performed preliminary cryo-EM and CLEM experiments. Autopsy material and associated clinical and neuropathological information was provided by J.R., T.L. 
and A.I., while S.C.F. did the pTDP-43 immunohistochemistry and immunofluorescence and Y.T.A. sampled the cases from the Queen Square Brain Bank. F.DG., F.I. and E.B. performed and analyzed inoculations on primary neurons. F.L., Z.M. and M.P wrote the manuscript. All authors read, edited and approved the final manuscript.

\section{Competing financial interests}

The authors declare no competing financial interests. 


\section{References}

1. Ling, S.C., Polymenidou, M. \& Cleveland, D.W. Converging mechanisms in ALS and FTD: disrupted RNA and protein homeostasis. Neuron 79, 416-438 (2013).

2. Neumann, M., et al. Ubiquitinated TDP-43 in frontotemporal lobar degeneration and amyotrophic lateral sclerosis. Science 314, 130-133 (2006).

3. Arai, T., et al. TDP-43 is a component of ubiquitin-positive tau-negative inclusions in frontotemporal lobar degeneration and amyotrophic lateral sclerosis. Biochem Biophys Res Commun 351, 602-611 (2006).

4. Buratti, E., et al. Nuclear factor TDP-43 and SR proteins promote in vitro and in vivo CFTR exon 9 skipping. EMBO J 20, 1774-1784 (2001).

5. Polymenidou, M., et al. Long pre-mRNA depletion and RNA missplicing contribute to neuronal vulnerability from loss of TDP-43. Nat Neurosci 14, 459-468 (2011).

6. Tollervey, J.R., et al. Characterizing the RNA targets and position-dependent splicing regulation by TDP-43. Nat Neurosci 14, 452-458 (2011).

7. Afroz, T., et al. Functional and dynamic polymerization of the ALS-linked protein TDP-43 antagonizes its pathologic aggregation. Nat Commun 8, 45 (2017).

8. Jiang, L.L., et al. The N-terminal dimerization is required for TDP-43 splicing activity. Sci Rep 7, 6196 (2017).

9. Gu, J., et al. Transactive response DNA-binding protein 43 (TDP-43) regulates alternative splicing of tau exon 10: Implications for the pathogenesis of tauopathies. J Biol Chem 292, 10600-10612 (2017).

10. Ederle, H. \& Dormann, D. TDP-43 and FUS en route from the nucleus to the cytoplasm. FEBS Lett 591, 1489-1507 (2017).

11. Dewey, C.M., et al. TDP-43 is directed to stress granules by sorbitol, a novel physiological osmotic and oxidative stressor. Mol Cell Biol 31, 1098-1108 (2011).

12. Alami, N.H., et al. Axonal transport of TDP-43 mRNA granules is impaired by ALS-causing mutations. Neuron 81, 536-543 (2014).

13. Gopal, P.P., Nirschl, J.J., Klinman, E. \& Holzbaur, E.L. Amyotrophic lateral sclerosis-linked mutations increase the viscosity of liquid-like TDP-43 RNP granules in neurons. Proc Natl Acad Sci U S A 114, E2466-E2475 (2017).

14. Kato, M., et al. Cell-free formation of RNA granules: low complexity sequence domains form dynamic fibers within hydrogels. Cell 149, 753-767 (2012).

15. Molliex, A., et al. Phase separation by low complexity domains promotes stress granule assembly and drives pathological fibrillization. Cell 163, 123-133 (2015).

16. Igaz, L.M., et al. Enrichment of C-terminal fragments in TAR DNA-binding protein-43 cytoplasmic inclusions in brain but not in spinal cord of frontotemporal lobar degeneration and amyotrophic lateral sclerosis. Am J Pathol 173, 182-194 (2008).

17. Neumann, M., et al. Phosphorylation of S409/410 of TDP-43 is a consistent feature in all sporadic and familial forms of TDP-43 proteinopathies. Acta Neuropathol 117, 137-149 (2009).

18. Lashley, T., Rohrer, J.D., Mead, S. \& Revesz, T. Review: an update on clinical, genetic and pathological aspects of frontotemporal lobar degenerations. Neuropathol Appl Neurobiol 41, 858-881 (2015).

19. Neary, D., et al. Frontotemporal lobar degeneration: a consensus on clinical diagnostic criteria. Neurology 51, 1546-1554 (1998).

20. Mackenzie, I.R. \& Neumann, M. Reappraisal of TDP-43 pathology in FTLD-U subtypes. Acta Neuropathol 134, 79-96 (2017).

21. Lee, E.B., et al. Expansion of the classification of FTLD-TDP: distinct pathology associated with rapidly progressive frontotemporal degeneration. Acta Neuropathol 134, 65-78 (2017).

22. Nonaka, T., et al. Prion-like properties of pathological TDP-43 aggregates from diseased brains. Cell Rep 4, 124-134 (2013). 
23. Tsuji, H., et al. Molecular analysis and biochemical classification of TDP-43 proteinopathy. Brain 135, 3380-3391 (2012).

24. Laferriere, F., et al. Quaternary structure of pathological prion protein as a determining factor of strain-specific prion replication dynamics. PLoS Pathog 9, e1003702 (2013).

25. Polymenidou, M., et al. Coexistence of multiple PrPSc types in individuals with CreutzfeldtJakob disease. Lancet Neurol 4, 805-814 (2005).

26. Carra, S., et al. Alteration of protein folding and degradation in motor neuron diseases: Implications and protective functions of small heat shock proteins. Prog Neurobiol 97, 83-100 (2012).

27. Neumann, M., et al. Absence of heterogeneous nuclear ribonucleoproteins and survival motor neuron protein in TDP-43 positive inclusions in frontotemporal lobar degeneration. Acta Neuropathol 113, 543-548 (2007).

28. Kametani, F., et al. Mass spectrometric analysis of accumulated TDP-43 in amyotrophic lateral sclerosis brains. Sci Rep 6, 23281 (2016).

29. Ingre, C., et al. A novel phosphorylation site mutation in profilin 1 revealed in a large screen of US, Nordic, and German amyotrophic lateral sclerosis/frontotemporal dementia cohorts. Neurobiol Aging 34, 1708 e1701-1706 (2013).

30. $\mathrm{Wu}, \mathrm{C} . \mathrm{H}$., et al. Mutations in the profilin 1 gene cause familial amyotrophic lateral sclerosis. Nature 488, 499-503 (2012).

31. Zhou, J., et al. Spinal muscular atrophy associated with progressive myoclonic epilepsy is caused by mutations in ASAH1. Am J Hum Genet 91, 5-14 (2012).

32. Xu, G., Stevens, S.M., Jr., Moore, B.D., McClung, S. \& Borchelt, D.R. Cytosolic proteins lose solubility as amyloid deposits in a transgenic mouse model of Alzheimer-type amyloidosis. Hum Mol Genet 22, 2765-2774 (2013).

33. McGurk, L., et al. Poly-A binding protein-1 localization to a subset of TDP-43 inclusions in amyotrophic lateral sclerosis occurs more frequently in patients harboring an expansion in C9orf72. J Neuropathol Exp Neurol 73, 837-845 (2014).

34. Kerman, A., et al. Amyotrophic lateral sclerosis is a non-amyloid disease in which extensive misfolding of SOD1 is unique to the familial form. Acta Neuropathol 119, 335-344 (2010).

35. Robinson, J.L., et al. TDP-43 skeins show properties of amyloid in a subset of ALS cases. Acta Neuropathol 125, 121-131 (2013).

36. Lin, W.L. \& Dickson, D.W. Ultrastructural localization of TDP-43 in filamentous neuronal inclusions in various neurodegenerative diseases. Acta Neuropathol 116, 205-213 (2008).

37. Guenther, E.L., et al. Atomic structures of TDP-43 LCD segments and insights into reversible or pathogenic aggregation. Nat Struct Mol Biol 25, 463-471 (2018).

38. Aguzzi, A., Heikenwalder, M. \& Polymenidou, M. Insights into prion strains and neurotoxicity. Nat Rev Mol Cell Biol 8, 552-561 (2007).

39. Yagi, H., et al. Zonisamide Enhances Neurite Elongation of Primary Motor Neurons and Facilitates Peripheral Nerve Regeneration In Vitro and in a Mouse Model. PLoS One 10, e0142786 (2015).

40. Danzer, K.M., Krebs, S.K., Wolff, M., Birk, G. \& Hengerer, B. Seeding induced by alphasynuclein oligomers provides evidence for spreading of alpha-synuclein pathology. $J$ Neurochem 111, 192-203 (2009).

41. Polymenidou, M. \& Cleveland, D.W. Biological Spectrum of Amyotrophic Lateral Sclerosis Prions. Cold Spring Harb Perspect Med (2017).

42. Polymenidou, M. \& Cleveland, D.W. The seeds of neurodegeneration: prion-like spreading in ALS. Cell 147, 498-508 (2011).

43. Sanders, D.W., et al. Distinct tau prion strains propagate in cells and mice and define different tauopathies. Neuron 82, 1271-1288 (2014).

44. Peelaerts, W., et al. alpha-Synuclein strains cause distinct synucleinopathies after local and systemic administration. Nature 522, 340-344 (2015). 
45. Meyer-Luehmann, M., et al. Exogenous induction of cerebral beta-amyloidogenesis is governed by agent and host. Science 313, 1781-1784 (2006).

Figure legends (max 250 words/legend)

Figure 1: SarkoSpin physically separates pathological TDP-43 from the majority of normal proteins in FTLD and ALS brains

a. Schematic representation of SarkoSpin protocol, including the number of autopsy samples from each disease type used in this figure. Samples were homogenized and solubilized with sarkosyl at $37^{\circ} \mathrm{C}$ in presence of Benzonase prior to a single centrifugation step to separate supernatant and pellet fractions. b-j. Biochemical analysis of SarkoSpin fractions extracted from human brain samples. Supernatants and pellets were subjected to SDS-PAGE followed by silver staining of total protein (c), or immunoblotted against total TDP-43 (b, e), phosphorylated TDP-43 at position S409/410 (pTDP43) (g), or hnRNPA1, hnRNPH, FUS, SOD1, Histone H3 and actin antibodies (i). Physiological TDP43 was found exclusively in supernatants for all patients, while the pathological species marked by phosphorylation were pelleted for all disease types, but are absent in controls. Pathological posttranslational modifications (PTMs) of TDP-43 were observed specifically in SarkoSpin pellets of disease cases: poly-ubiquitination smear $\left(^{* * *}\right)$, hyper-phosphorylation of TDP-43 detected by both anti-pTDP-43 (S409/410) and anti-TDP-43 (total) as a slower migrating band at $45 \mathrm{kDa}\left({ }^{* *}\right)$ and the Cterminal fragment of $25 \mathrm{kDa}\left({ }^{*}\right)$. d,f,h,j. Quantifications of the percentage of each protein residing in SarkoSpin supernatant or pellet for all patients used in this experiment. S - supernatant, $\mathrm{P}$ - pellet. Bars represent mean with standard deviations. $n=6$ biologically independent human brain samples for control, ALS, FTLD-TDP-A and FTLD-TDP-C groups. The results shown in b, c, e, g, i were repeated twice with all 27 independent biological samples with similar results, quantifications of one experiment are shown in $\mathbf{d}, \mathbf{f}, \mathbf{h}, \mathbf{j}$. 
Figure 2: SarkoSpin extracts from different disease subtypes have distinct protein compositions beyond TDP-43

a. Overlap of proteins with the highest values of idealized vector correlation when comparing each ALS, FTLD-TDP-A, and FTLD-TDP-C to controls (Pearson's correlation method). Only proteins with correlation values $>0.5$ were taken into account. Proteins further validated by immunoblot and immunohistochemistry are highlighted in bold. b. Representative immunoblots on SarkoSpin pellets probed against several mass spectrometry hits - PFN1, ASAH1 and TXNL1, showing their enrichment in the insoluble fraction in respective disease subtypes. c. Correlation dot plots of pTDP43 levels and insolubility of selected mass spectrometry candidates analyzed in b. (Pearson's correlation method). d. Representative immunoblots on SarkoSpin pellet and supernatant fractions probed with ubiquitin antibody, indicating enrichment of ubiquitinated pathological assemblies in the SarkoSpin pellets fractions. e. Correlation dot plot of pTDP-43 and ubiquitin levels in SarkoSpin pellets across disease subtypes (Pearson's correlation method). f. Co-immunofluorescence of pTDP43 with ubiquitin in FTLD-TDP-A and FTLD-TDP-C post mortem brain sections. g. Quantification of aggregates double-positive for pTDP-43 and ubiquitin in FTLD-TDP-A and FTLD-TDP-C (dots represent single \% values quantified per each image; error bars show mean with SD, unpaired twosided $t$ test with $\left.p=1.64 \times 10^{-6}\right)$. $h$. Representative images of co-immunofluorescence of GFAP marking astrocytes with a mass spectrometry hit FBXO2. In panels $\mathbf{f}$ and $\mathbf{h}$ the images are overlaid with DAPI nuclear staining in blue. For $\mathrm{c}$, e and $\mathrm{g}, \mathrm{n}=4$ biologically independent human brain samples for ALS, FTLD-TDP-A and FTLD-TDP-C groups. In b, $d$, f and h, results were repeated twice independently for all $n=4$ independent biological samples of each disease group with similar results.

Figure 3: Diverse size distributions of TDP-43 aggregates isolated from different types of TDP-43 proteinopathies

a. Schematic representation of the velocity sedimentation protocol. After SarkoSpin solubilization, brain homogenates were loaded on top of iodixanol gradients and fractionated by sedimentation 
velocity upon ultracentrifugation. b. Representative immunoblots of the velocity sedimentation profiles of physiological and pathological TDP-43 in various disease types. SarkoSpin-solubilized brain homogenates from frontal and motor cortex of control (black), ALS (red), FTLD-TDP-A (green), and FTLD-TDP-C (blue) were fractionated by velocity sedimentation and the distribution of total TDP-43 (top panels, immunoblots) and of pTDP-43 (bottom panels, dot blots) was analyzed in the collected fractions (numbered from top to bottom of the gradient). Immunoblots were repeated three times independently with similar results. c-d. The relative amounts of TDP-43 (c), and pTDP-43 (d) per fraction were quantified from control (black, $n=6$ ), ALS (red, $n=4)$, FTLD-TDP A (green, $n=10$ ), FTLDTDP $C$ (blue, $n=8$ ) velocity sedimentation fractionations, with $n$ being the number of biologically independent human brain samples. Data presented are the mean curves (bold lines) with standard deviations (lighter shade areas) for each patient group. Panels below the graphs show the statistical significance of the difference in TDP-43 partition in each fraction based on two-ways ANOVA followed by Tuckey's multiple-comparison tests.

Figure 4: Distinct density profiles of TDP-43 aggregates isolated from different types of TDP-43 proteinopathies

a. Schematic representation of the density floatation protocol. Briefly, after SarkoSpin solubilization, brain homogenates were loaded in the middle of iodixanol gradients and fractionated by density upon isopycnic equilibrium ultracentrifugation. b. Representative immunoblots of the density sedimentation profiles of physiological and pathological TDP-43 in various disease types. SarkoSpin-solubilized brain homogenates from frontal and motor cortex of control (black), ALS (red), FTLD-TDP-A (green), and FTLD-TDP-C (blue) were fractionated by density and the distribution of total TDP-43 (top panels, immunoblots) and of pTDP-43 (bottom panels, dot blots) was analyzed in the collected fractions (numbered from top to bottom of the gradient). Density values calculated by refractometry are indicated on the top of the graph. Immunoblots were repeated three times independently with similar results. c-d. The relative amounts of TDP-43 (c), and pTDP-43 (d) per fraction were quantified from 
all immunoblots of control (black, $n=6$ ), ALS (red, $n=4$ ), FTLD-TDP-A (green, $n=10$ ), FTLD-TDP-C (blue, $n=8$ ) density fractionations, with $\mathrm{n}$ being the number of biologically independent human brain samples. Data presented are the mean curves (bold lines), with standard deviations (lighter shade areas) for each group of patients. Panels below the graphs show the statistical significance of the difference in TDP-43 partition in each fraction based on two-ways ANOVA followed by Tuckey's multiple-comparison tests.

Figure 5: Distinct structure of pathological TDP-43 isolated from different disease subtypes

a. Transmission Electron Microscopy (TEM) images of SarkoSpin-extracted aggregates from control (black), FTLD-TDP type A (green) and FTLD-TDP type C (blue) immunolabeled with monoclonal TDP-43 antibody (epitope: 203-209). Red arrows point to gold-positive structures, yellow arrows to lipid contaminants, orange arrows to proteinaceous filamentous structures. b. Part of the FTLD-TDPC image shown in panel a was band-pass filtered. The scale bar is $100 \mathrm{~nm}$ c. TEM images of SarkoSpin-extracted aggregates from FTLD-TDP-A patient immunogold labeled against TDP-43, without embedding in methylcellulose (MC). d. Proteolytic profiles of pathological TDP-43 from different disease subtypes, as annotated. Two controls and four of each disease samples were incubated with either $10 \mu \mathrm{g} / \mathrm{mL}$ Chymotrypsin or $100 \mu \mathrm{g} / \mathrm{mL}$ Trypsin for $30 \mathrm{~min}$ at $37^{\circ} \mathrm{C}$ during SarkoSpin solubilization. Pellets were immunoblotted with anti-TDP-43 antibodies. As reference to antigens and epitopes of used antibodies, TDP-43 secondary structure is represented below. NTD N-terminal domain; NLS - nuclear localization signal; RRM1 and 2 - RNA-recognition motifs; LCD low-complexity domain; Phosphorylation is marked at the very C-terminus. e. Dot plot representing the levels of pTDP-43 Chymotrypsin and Trypsin-resistant fragments normalized to total level of undigested TDP-43 species across disease subtypes. $n=4$ biologically independent human brain samples per disease group and per protease used. Dots represent single analyzed patients per disease subtype; error bars show mean with SD (unpaired two-sided t tests). f. Representative TEM images of trypsinized SarkoSpin-extracted aggregates from FTLD-TDP type A and type C 
immunogold-labeled against pTDP-43 pS409/410. Red arrows point to gold-positive structures. Experiments were repeated two (d), three $(\mathbf{c}, \mathbf{f})$, and four $(\mathbf{a}, \mathbf{b})$ independent times with similar results.

Figure 6: Differential cyto- and neurotoxicity of FTLD-TDP type A and type $C$ pathological assemblies a. Schematic representation of SarkoSpin inoculations on arrested HEK293 cells expressing TDP-43HA. b. Viability ratio of cells with induced expression of TDP-43-HA (+DOX) and without it (-DOX). Each point represents the viability ratio of cells inoculated with the SarkoSpin pellet of a single patient; $n=6, n=9$ and $n=7$ biologically independent human brain samples for control, FTLD-TDP-A and FTLDTDP-C groups respectively, Bars show mean \pm SD, after normal distribution and equal variance verifications (Shapiro-Wilk and F-test), two-sided unpaired $t$ test gave the indicated $p$ value. c. Immunoblots of SarkoSpin supernatants and pellets from TDP-43-HA-expressing cells inoculated with SarkoSpin pellet pools extracted from control $(n=3)$, FTLD-TDP-A $(n=3)$ or FTLD-TDP-C $(n=3)$ patients. Full-size blots are shown in Supplementary Fig. 18h. d. Schematic representation of neurite outgrowth and toxicity assay on mouse primary cortical neuronal cultures upon inoculations of SarkoSpin pellets from different disease subtypes. e-h $n=3$ biologically independent human brain samples for control (black), FTLD-TDP-A (green) and FTLD-TDP-C (blue), used for cell inoculations in three independent experiments. e. Representative fluorescence calcein (green, upper rows) images and corresponding neurite and cell body segments (blue and yellow respectively, lower rows) for control (upper panel), FTLD-TDP-A (middle panel) and FTLD-TDP-C (lower panel) SarkoSpin pellet inoculations at indicated total protein amounts at 10 DIV endpoint. f. Dynamics of neurite outgrowth revealed by quantification of the phase contrast images of inoculated neurons at $0.3 \mu \mathrm{g}$ total protein dose per well (error bars show mean and SD). g-h. Quantification of the endpoint calcein fluorescence images using a cell body $(\mathbf{g})$ or neurite length $(\mathbf{h})$ segmentation algorithm for different doses of SarkoSpin extracts (error bars show mean and SD). For $\mathbf{c}$ and e, experiments were independently repeated two and three times respectively with similar results. 


\section{Materials and methods}

\section{Cases}

No statistical methods were used to pre-determine sample sizes. Instead, we used the maximum number of samples that were available to us. Brains were donated to the Queen Square Brain Bank for Neurological disorders, Department of Movement Disorders, UCL Institute of Neurology, London, UK, or the Department of Neurology, University of California, San Diego, CA, USA, or the Netherlands Brain Bank, Netherlands Institute for Neuroscience, Amsterdam, Netherlands (open access: www.brainbank.nl). All patient material has been collected from donors for or from whom a written informed consent for a brain autopsy and the use of the material and clinical information for research purposes has been obtained by the respective institution.

\section{Antibodies}

All antibodies used are listed with suppliers, clone or catalog numbers and dilutions used in Supplementary Table 2. Each antibody was validated for the species (human, mouse) and application (WB, DB, IF, IHC) by the correspondent manufacturer.

\section{Immunohistochemistry}

Fresh tissue from frontal cortex of FTLD-TDP-A cases, either sporadic or with C9ORF72 mutation, as well as a FTLD-TDP-C cases were fixed with phosphate-buffered $3.65 \%$ formaldehyde, embedded in paraffin, cut into $6 \mu \mathrm{m}$ serial sections and mounted on glass slides. Sections were deparaffinized and rehydrated using a gradient of alcohol. Antigen retrieval was done by boiling the sections in $10 \mathrm{mM}$ citrate buffer ( $\mathrm{pH} 6.0$ ) in a microwave oven. Immunohistochemistry was performed using the avidinbiotin complex (ABC) detection system (Vector Laboratories) and 3,3-diaminobenzidine. Endogenous peroxidases were first quenched with $5 \% \mathrm{H}_{2} \mathrm{O}_{2}$ in methanol for 30 minutes, and sections were blocked in $0.1 \mathrm{~mol} / \mathrm{L}$ Tris with $2 \%$ fetal bovine serum for 5 minutes. Primary antibodies were incubated for 2 hours at room temperature. After washing, sections were sequentially incubated with biotinylated 
secondary antibodies for 1 hour and avidin-biotin complex for 1 hour. Bound antibody complexes were visualized by incubating sections in a solution containing $100 \mathrm{mM}$ Tris, $\mathrm{pH} 7.6,0.1 \%$ Triton $\mathrm{X}-100,1.4$ $\mathrm{mM}$ di-aminobenzidine, $10 \mathrm{mM}$ imidazole, and $8.8 \mathrm{mM} \mathrm{H} \mathrm{H}_{2} \mathrm{O}_{2}$. Sections were then lightly counterstained with hematoxylin, dehydrated, and coverslipped. Digital images were obtained using Life Technologies EVOS FL Auto imaging system (Life Technologies).

All antibodies used for immunofluorescence and western blot are listed in Supplementary Table 2 with the respective catalogue numbers and working dilutions indicated for each assay.

\section{Homogenization of brain tissue}

Frozen tissue from motor or frontal cortex of all groups of patients were homogenized at $20 \% \mathrm{w} / \mathrm{v}$ in homogenization - solubilization buffer (HS) without detergent: $10 \mathrm{mM}$ Tris $\mathrm{pH} 7.5,150 \mathrm{mM} \mathrm{NaCl}, 0.1$ mM EDTA, $1 \mathrm{mM}$ DTT, Complete EDTA-free protease inhibitors (Roche) and PhosSTOP phosphatase inhibitors (Roche). Samples were subjected to three rounds of 30 seconds homogenization in tubes containing a mixture of ceramic beads of 1.4 and $2.8 \mathrm{~mm}$ of diameter on a Minilys device (Bertin) at full speed with cooling on ice between each round. Samples were aliquoted, snap frozen in liquid nitrogen and kept in $-80^{\circ} \mathrm{C}$ before use.

\section{SarkoSpin on patient tissues}

All SarkoSpin steps were done in $1.5 \mathrm{~mL}$ Protein Low Binding tubes (Eppendorf). Brain homogenate aliquots of $150 \mu \mathrm{L}$ were thawed on ice and equalized for protein concentration in final volume of 200

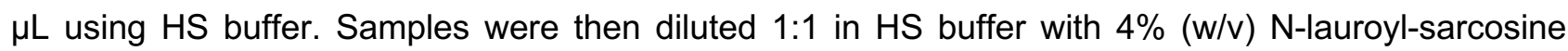
(sarkosyl, Sigma), $2 \mathrm{U} / \mu \mathrm{L}$ Benzonase (Novagen) and $4 \mathrm{mM} \mathrm{MgCl}_{2}$, reaching final volume of $400 \mu \mathrm{L}$ with $2 \%$ Sarkosyl, $1 \mathrm{U} / \mu \mathrm{L}$ Benzonase and $2 \mathrm{mM} \mathrm{MgCl}_{2}$ final concentrations. SarkoSpin solubilization was then performed by incubating at $37^{\circ} \mathrm{C}$ under constant shaking at $600 \mathrm{rpm}$ (Thermomixer, Eppendorf) for 45 minutes. Solubilized brain homogenates $(400 \mu \mathrm{L})$ were then further diluted by 
adding $200 \mu \mathrm{L}$ of ice-cold HS buffer containing $0.5 \% \mathrm{w} / \mathrm{v}$ sarkosyl prior to centrifugation at $21,200 \mathrm{~g}$ on a benchtop centrifuge (Eppendorf) for 30 minutes at room temperature. Alternatively, for EM sample preparation, to remove lipids from precipitating along with protein aggregates, solubilized material was overlaid on top of $400 \mu \mathrm{l}$ sucrose cushion ( $40 \% \mathrm{w} / \mathrm{v}$ sucrose (Sigma) in HS buffer containing $0.5 \% \mathrm{w} / \mathrm{v}$ sarkosyl) and centrifuged at $21,200 \mathrm{~g}$ for 1 hour at room temperature. For the analysis of proteolytic profiles, after the initial 15 minutes of SarkoSpin solubilization, the respective protease was added for the remaining 30 minutes of incubation in the following final concentrations: $100 \mu \mathrm{g} / \mathrm{mL}$ for Proteinase $\mathrm{K}, 10 \mu \mathrm{g} / \mathrm{mL}$ for Chymotrypsin or $100 \mu \mathrm{g} / \mathrm{mL}$ for Trypsin (from stock solutions of $2 \mathrm{mg} / \mathrm{mL}, 0.2$ $\mathrm{mg} / \mathrm{mL}$ or $2 \mathrm{mg} / \mathrm{mL}$, respectively). Supernatants (approx. $600 \mu \mathrm{L}$ ) were collected in a new tube and pellets were resuspended in desired volume of HS buffer containing $0.5 \%$ w/v sarkosyl for further analysis, including dot blot, SDS-PAGE and immunoblot or native-PAGE. Whenever specified, the fractions were passed through low protein binding $0.22 \mu \mathrm{m}$ filters (Millex-GV) to remove large nonsolubilized particles.

\section{SarkoSpin on cells}

Transfected NSC-34 cells in $10 \mathrm{~cm}$ dishes (see below) were lysed in $400 \mu \mathrm{L}$ of ice-cold $1 \mathrm{X}$ HS buffer with $0.5 \%$ sarkosyl, $2 \mathrm{mM} \mathrm{MgCl}_{2}$ and $\sim 100 \cup$ Benzonase, and $60 \mu \mathrm{L}$ of the lysate was put aside for protein concentration measurements and immunoblots. For SarkoSpin of inoculated HEK cells, cells plated in a 6-well plate were lysed in $170 \mu$ of ice-cold $1 \mathrm{X}$ HS buffer with $0.5 \%$ sarkosyl, $2 \mathrm{mM} \mathrm{MgCl}_{2}$ and $\sim 25 \mathrm{U}$ Benzonase per well. For sample solubilization, to aliquots of $170 \mu \mathrm{L}$ of lysate, $52 \mu \mathrm{L} 1 \mathrm{X} \mathrm{HS}$ and $178 \mu \mathrm{L} 2 \mathrm{X}$ HS with $4 \%$ sarkosyl were added, for a final concentration of $2 \%$ sarkosyl in $400 \mu \mathrm{L}$ of sample. After a 30-minute incubation on ice with vortexing every 10 minutes, the samples were diluted by adding $200 \mu \mathrm{L}$ of ice-cold HS buffer and centrifuged as described before for SarkoSpin on brain homogenates. Supernatants were collected in a new tube and pellets were resuspended in $1 \mathrm{X} \mathrm{HSI}$ buffer with $0.5 \%$ sarkosyl for immunoblot samples or in PBS for inoculation experiments in HEK cells. 
SarkoSpin fractions from cell line experiments were processed for immunoblot as described before for brain homogenates.

\section{Mass Spectrometry}

After clean-up with C18 cartridges, the peptide samples were measured on a TripleTOF 5600 instrument ( $\mathrm{BB}$ Sciex, Concord $\mathrm{ON}$ ) equipped with a nano-electrospray ion source and an Eksigent 1D-plus liquid chromatography system (Eksigent, Dublin, CA) or a $Q$ Exactive Plus instrument (Thermo Fisher) equipped with an EASY-nLC 1000 (Thermo Fisher). For the TripleTOF measurements, peptides were loaded onto a $20-\mathrm{cm}$ long, $75 \mu \mathrm{m}$ i.d. PicoFrit Column (New Objective, Woburn, MA) packed in-house with Magic C18 AQ $3 \mu \mathrm{m}$ beads (Michrom Bioresources, Auburn, CA) and separated with a linear gradient from $5 \%$ to $35 \%$ acetonitrile in $90 \mathrm{~min}$ at a flowrate of $300 \mathrm{~nL} / \mathrm{min}$. Survey scans were acquired in $300 \mathrm{~ms}$ and up to 20 product ion scans were collected in $75 \mathrm{~ms}$ if exceeding a threshold 150 counts per second with a charge state from $2+$ to $5+$. For the $\mathrm{Q}$ Exactive Plus measurements, peptides were loaded on a $40 \mathrm{~cm}$ long, $75 \mu \mathrm{m}$ i.d. column packed in-house with ReproSil-Pur C18-AQ resin (1.9 $\mu \mathrm{m}$, Dr. Maisch). Peptides were eluted for 50 min using a segmented linear gradient of $5 \%$ to $40 \%$ acetonitrile at a flow-rate of $300 \mathrm{~nL} / \mathrm{min}$. Survey full-scan mass spectra were acquired with mass range $350-1500 \mathrm{~m} / \mathrm{z}$, at a resolution of 70,000 at $200 \mathrm{~m} / \mathrm{z}$ and the 20 most intense ions above an intensity of $3.6 \mathrm{e} 4$ were sequentially isolated, fragmented (HCD at $25 \mathrm{NCE}$ ) and measured at a resolution of 17,500 at $200 \mathrm{~m} / \mathrm{z}$. Peptides with a charge of +1 or with unassigned charge state were excluded from fragmentation for MS2, and a dynamic exclusion of $30 \mathrm{~s}$ was applied. Ions were accumulated to a target value of $3 e 6$ for MS1 and of 1e5 for MS2.

MGF-peak lists generated from the acquired spectra by ProteinPilot from the TripleTOF runs and .RAW files from the $Q$ Exactive Plus runs were searched against a human Uniprot protein database (68978 sequence) by Sequest HT in Proteome Discoverer 1.4 (Thermo Scientific, San Jose, CA) with enzymatic specificity trypsin, precursor mass tolerance $20 \mathrm{ppm}$ and fragment tolerance $0.1 \mathrm{Da}$ and $0.02 \mathrm{Da}$ for TripleTOF and Q Exactive Plus, respectively. Methionine oxidation and 
carbamidomethylation of cysteines were set as respectively variable and static modifications. Percolator was used to filter the results to a $1 \%$ peptide false discovery rate. Spectral counting was used for quantifying protein differences between samples.

\section{Statistical Analysis of Mass Spectrometry Data}

Unless stated otherwise the higher-level data analysis was carried out using R/Bioconductor ( $\mathrm{R}$ Core Team, 2017). The count values for each sample were normalized by dividing the values by the respective trimmed mean (removing the highest and lowest $5 \%$ values). As the samples were run in two separate batches, the ComBat method ${ }^{46}$ was applied to correct for the batch effect. To identify proteins that were most consistently enriched in patient samples, we established an idealized vector correlation method: a vector of the same number of 0 s as control samples and the same number of $1 \mathrm{~s}$ patient samples was created. For each protein, we measured the correlation of this idealized vector to the vector of normalized and batch-corrected protein abundance values of control and patient samples. We then selected the 50 best-correlated proteins and established a heatmap of the log2 abundance values. For the heatmap, we applied a median per protein normalization, by dividing the values of each protein by the respective median value of the control samples.

\section{Validation of Mass Spectrometry hits by immunofluorescence}

Formalin fixed paraffin embedded tissue was sectioned at $7 \mu \mathrm{m}$. Sections were deparaffinized in xylene and rehydrated using a gradient of alcohol. Endogenous peroxidases were first quenched with $0.3 \% \mathrm{H}_{2} \mathrm{O}_{2}$ in methanol for 10 minutes. Antigen retrieval was undertaken in a pressure cooker for 10 minutes at maximum pressure in $10 \mathrm{mM}$ citrate buffer $(\mathrm{pH} \mathrm{6.0)}$ and sections were blocked in $10 \%$ non-fat milk for 30 minutes. Sections were incubated with phosphorylation-specific rabbit polyclonal pAb anti-pTDP (pS409 1:20,000 Cosmobio). After washing, sections were sequentially incubated with biotinylated swine anti-rabbit secondary antibody for 30 minutes and avidin-biotin complex for 30 minutes. pTDP-43 was visualized using TSA Rhodamine amplification (Perkin-Elmer) at 1:500 for 30 
minutes. Sections were then incubated with antibodies raised against either TLXN1, FBXO2, ASAH1 followed by incubation in AlexaFluor 468 at 1:1000 for 1 hour at room temperature. Sections were viewed and imaged with a Leica TCS4D using a 3-channel scan head fluorescent microscope and image deconvoluted using Leica software.

\section{Velocity sedimentation and density floatation gradients}

Brain homogenates were thawed on ice and solubilized as described previously (SarkoSpin). For velocity sedimentations, a volume of $200 \mu \mathrm{L}$ was loaded on top of a $4 \mathrm{~mL}$ continuous $10-25 \%$ iodixanol gradient (Optiprep, Sigma) in HS buffer with $0.5 \% \mathrm{w} / \mathrm{v}$ sarkosyl linearized directly in ultracentrifuge $4.2 \mathrm{~mL}$ tubes (Seton) with a Gradient Master (Biocomp). For density floatation gradients, $220 \mu \mathrm{L}$ of HS buffer with $40 \%$ iodixanol and $0.5 \%$ w/v sarkosyl was loaded within a $4 \mathrm{~mL}$ $10-60 \%$ discontinuous iodixanol gradient in HS buffer with $0.5 \% \mathrm{w} / \mathrm{v}$ sarkosyl. The gradients were centrifuged at $310,000 \mathrm{~g}$ for 45 min (velocity) or at $125,000 \mathrm{~g}$ for 17 hours (density) in a swingingbucket SW-60 Ti rotor using an Optima XPN-100 ultracentrifuge (Beckman Coulter). Gradients were then segregated into 16 equal fractions from the top using a piston fractionator (Biocomp) and a fraction collector (Gilson). Fractions were aliquoted for further analysis of their content by dot blot, immunoblot on SDS-PAGE or native-PAGE. Gradient linearity was verified by refractometry.

\section{Analysis of protein content of SarkoSpin, velocity and density fractions}

Aliquots of the collected fractions were mixed with SDS-PAGE loading buffer with reducing agent (Life Technologies) and denatured at $95^{\circ} \mathrm{C}$ for 5 minutes, prior to migration on Bolt $12 \%$ Bis-Tris gels (Life Technologies). For visualizing total proteins, gels were stained with silver nitrate. For immunoblots, gels were electro-transferred onto nitrocellulose membranes with iBlot 2 (Life Technologies). For dot blotting, native fractions were spotted onto nitrocellulose $0.2 \mu \mathrm{m}$ membranes using a dot blot vacuum device (Whatman). Nitrocellulose membranes were blocked with $5 \% \mathrm{w} / \mathrm{v}$ skimmed powder milk (or 5 $\%$ w/v BSA for pS409/410 antibody) in PBS-Tween and probed with primary antibodies 
(Supplementary Table 2). Immunoreactivity was visualized by chemiluminescence (GE Healthcare). The amount of the respective protein in each fraction was determined by the Image Studio Lite software, after acquisition of chemiluminescent signals with a Fuji LAS 4000 digital imager (GE Healthcare). Profiles obtained by immunoblot were normalized and plotted with SD, with all respective student test and ANOVA using the Prism software. Fractions were also subjected to native-PAGE immunoblotting, for which aliquots were mixed with native sample buffer and G250 additive (Life Technologies) and resolved using 3-12\% Bis-Tris Native-PAGE gels (Life Technologies) and NativeMark protein ladder (Life Techologies). Gels were incubated with $2 x$ transfer buffer and electrotransferred with iBlot 2 on PVDF membranes. After fixation with $8 \%$ acetic acid, air-drying and reactivation with methanol, membranes were immunoblotted and probed as described above.

\section{Transmission Electron Microscopy}

SarkoSpin pellets from different disease types and control patients were resuspended in water and passed through protein low binding $0.22 \mu \mathrm{m}$ syringe filters. The resulting filtrates were fixed with glutaraldehyde water solution (EMS \#16220) (final concentration of $0.05 \%$ ) and subjected to immunogold labeling. A 300 mesh copper grid (Plano \#G2300C), formvar coated and glow discharged, was put on a $3 \mu \mathrm{L}$ drop of fixed sample for 3 minutes. The grid was then washed 3 times 20 seconds in aldehyde blocking solution with $0.15 \%$ Glycine (Sigma \#G7126) in PBS and 3 times 20 seconds in PBS, followed by 5 minute incubation in blocking solution (PBG): PBS with $0.2 \%$ gelatin (Sigma \#G6650) and 0.5 \% BSA (Applichem \#A6588). The grid was probed with TDP-43 60019-2-lg (mouse 1:50 in PBG solution, Proteintech) for 20 minutes (the negative control without primary antibody was incubated instead in PBG solution). The grid was washed in PBG solution 3 times 20 seconds, and incubated for another 5 minutes in PBG, before probing with secondary antibody anti-mouse gold 12 nm gold (1:20 in PBG solution, Jackson ImmunoResearch \#115-205-146) for 20 minutes. The grid was washed 4 times 20 seconds in PBG and twice 20 seconds in PBS, followed by a second fixation step for 30 seconds in $0.1 \%$ glutaraldehyde in PBS and 4 times 20 seconds washed in water. The 
grid was then counterstained with $1 \%$ uranyl acetate (Fluka \#73943) in water for 1 minute. Excess solution was sucked off using filter paper and the grid was air-dried. Alternatively, to avoid drying artifacts, the counterstaining solution included $1.8 \%$ methylcellulose (Sigma) in water and $0.3 \%$ uranyl acetate in water (incubation for 3 minutes staining procedure on ice). Lastly, the grid was picked up with a loop, excess solution was sucked off with filter paper, air-dried, and the specimen imaged with a CM100 transmission electron microscope (Thermo Fisher Scientific, Eindhoven, The Netherlands) at an acceleration voltage of $80 \mathrm{kV}$ using a Gatan Orius 1000 digital camera (Gatan, Munich, Germany).

\section{Plasmid generation}

A multiply-tagged TDP-43-encoding pcDNA5 plasmid $^{47}$ was modified in order to obtain a sequence coding for a single C-terminally HA-tagged TDP-43. The N-terminal GFP, His6 and myc (2) tags and the residual C-terminal amino acids between the HA tag and the stop codon were deleted in two subsequent reactions using the Q5 Site-Directed Mutagenesis Kit (New England Biolabs, E0554S) according to the manufacturer's instructions, with the following primers: 5'-TCT GAA TAT ATT CGG GTA ACC GAA GAT GAG AAC GAT GAG-3', 5'- CAT CCG CGG GGC AGG GGT-3' (tags, Ta = 72 'C), 5'-TAA ACC CGC TGA TCA GCC-3', 5'-AGC ATA ATC AGG GAC ATC ATA AG-3' (residual amino acids, $\left.\mathrm{Ta}=63^{\circ} \mathrm{C}\right)$.

\section{Cell culture, stable cell line generation and transient transfection}

HEK293 Flp-In T-REx cells (Invitrogen, R78007) were cultured in DMEM (Sigma, D5671) supplemented with 10\% FBS (Gibco, 10270-106), 1X PenStrep (Gibco, 15140-122), 1X GlutaMAX (Gibco, 35050061), $15 \mu \mathrm{g} / \mathrm{mL}$ blasticidin S (Gibco, R21001; Invivogen, ant-bl-10p) and $100 \mu \mathrm{g} / \mathrm{mL}$ zeocin (Gibco, R25001). In order to generate a stable cell line expressing HA-tagged TDP-43, $3 \cdot 10^{5}$ cells were plated per well in a 6-well plate and transfected after 48 hours with 125 ng of pcDNA5TDP43-HA plasmid and $1125 \mathrm{ng}$ of pOG44 plasmid with $6.25 \mu \mathrm{L}$ Lipofectamine 2000 (Invitrogen, 
11668019) according to the manufacturer's instructions. After a 48-hour recovery, cells were passaged to a $10-\mathrm{cm}$ dish with same media as before with the substitution of zeocin for $100 \mu \mathrm{g} / \mathrm{mL}$ hygromycin (Gibco, 10687-010) for the selection of cells that integrated the TDP-43-HA encoding sequence in the genome. Cells were selected for 2-3 weeks before expansion and banking, and afterwards cultured in the same medium.

For production of SarkoSpin-insoluble phosphorylated GFP-TDP-43 aggregates, $2 \cdot 10^{6}$ motor neuronlike mouse NSC-34 cells were plated on a Matrigel-coated (Corning, 354234) $10 \mathrm{~cm}$ dish in differentiation media, consisting of DNEM/F-12 (Gibco, 21331-020) supplemented with 1X B27 supplement (Gibco, 17504-044), 1\% GlutaMAX, 1X N2 supplement (Gibco, 175020-01) and 0.1X PenStrep. After 48 hours, cells were transfected with $5 \mu \mathrm{g}$ of GFP-TDP-43-HA and $20 \mu \mathrm{L}$ Lipofectamine 2000 according to the manufacturer's instructions.

\section{Viability assay on HEK293 cells}

To study the toxicity of the SarkoSpin-insoluble pellets, $6 \cdot 10^{4} \mathrm{HEK} 293$ cells/well were plated on a Matrigel-coated 24-well plate and TDP-43-HA expression was induced after 24 hours with $1 \mu \mathrm{g} / \mathrm{mL}$ of doxycycline. 20 hours later, cells were mitotically arrested with $10 \mu \mathrm{M}$ AraC (Sigma, C1768), and after another 6-8 hours, transfected with freshly-prepared SarkoSpin pellet fractions. To prepare the inocula, SarkoSpin pellets were washed with $300 \mu \mathrm{l}$ of sterile PBS to remove traces of sarkosyl and remove the vast majority of lipid contaminants. Pellets were subsequently resuspended in $200 \mu$ l cellculture grade PBS and sonicated at $60 \%$ amplitude for 2 minutes of sonication time $(1 \mathrm{~s}$ ON $/ 1 \mathrm{~s}$ OFF pulses) in water-bath sonicator (Q500, QSonica). $20 \mu \mathrm{l}$ of sonicated material was transfected with 2.5 $\mu \mathrm{L}$ Lipofectamine in a total volume of $290 \mu \mathrm{L}$ OptiMEM per well. Additional $210 \mu \mathrm{L}$ of media was added 4 hours after protein transfection, followed by whole media change after 24 hours, with fresh doxycycline and AraC. For the viability measurements, on day 3 after lipofection, $55 \mu \mathrm{L}$ of $5 \mathrm{mg} / \mathrm{mL}$ Thiazolyl Blue Tetrazolium Bromide (Sigma, D5655) was added per well and incubated at $37^{\circ} \mathrm{C}$ for 1 hour. The reaction was stopped and the formazan solubilized with $100 \mu \mathrm{L}$ of a solution containing 
$16 \%$ sodium dodecyl sulfate (SDS), $40 \%$ dimethylformamide (DMF) and $2 \%$ glacial acetic acid. Absorption of the solubilized formazan at $570 \mathrm{~nm}$ was subtracted the absorption from cell debris and other contaminants at $630 \mathrm{~nm}$. The values of absorption in cells expressing TDP-43-HA were divided by the those of non-induced cells, and the value of the ratio of cells inoculated with SarkoSpin pellets from control patients set to one by dividing every value by the average ratio of all control conditions. Every condition was performed in triplicates.

\section{Immunocytochemistry}

TDP-43-HA-expressing HEK293 on microscopy 24-well plates (Ibidi, 82406) inoculated with GFPTDP-43-HA aggregates were wash once with PBS before fixation in 4\% formaldehyde (brand) for 10 minutes at room temperature. After washing twice with PBS, cells were permeabilized and blocked in blocking buffer (10\% donkey serum (Millipore) with 0.3\% Triton X-100 (Sigma) in PBS) for at least 30 minutes at room temperature. Primary antibodies against HA-tag (1:500, Cell Signalling Technologies) and GFP (1:1000, Rockland Immunochemicals) were incubated in blocking buffer overnight at $4{ }^{\circ} \mathrm{C}$. After three washes with PBS, secondary antibody Alexa-647 donkey anti-rabbit (Invitrogen, A-31573) was applied in PBS for 1 hour at room temperature, followed by three additional washes in PBS. Cells were covered with coverslips using ProLong Gold Antifade Mountant with DAPI (Invitrogen, P36931).

\section{Primary mouse cortical neuronal culture in 96-well plate}

Timed pregnant C57/BL6J female mice were received from Charles River Labs 2 days before initiation of the primary culture. Cortices were harvested from E18 mouse embryos and dissociated enzymatically and mechanically (using the neuronal tissue dissociation kit, C-Tubes, and an Octodissociator with heaters, Miltenyi Biotech, Germany) to yield a homogenous cell suspension. The cells were then plated at 20000 per well in 96-well plates in neuronal medium (Neuronal Macs medium, Miltenyi Biotech, Germany) containing 0.5\% Penicillin/Streptomycin, $0.5 \mathrm{mM}$ alanylglutamine, and 2\% Neurobrew supplement (Miltenyi Biotech, Germany). In such cultures, and in 
control conditions, neurons represented approximately $85-95 \%$ of the cell population, with astrocytes and oligodendrocytes reaching $5-15 \%$ (GFAP+ or CNPase+), while the microglia (Iba1+) were undetectable (not shown). Thus, in the text, and for the sake of simplicity, they are referred to as "neurons". After 4 days in vitro (DIV), Sarkospin pellet pools from 3 Control, 3 FTLD-TDP-A or 3 FTLD-TDP-C patients were resuspended in sterile PBS and sonicated with a cup horn sonicator (Q500, QSonica) at 100\% set amplitude for 4 minutes of 1 second pulse / 1 second pause, and added to the medium at four different dilutions after normalization based on BCA-measured protein concentrations $(1,0.3,0.1,0.03 \mu \mathrm{g}$ of total protein amount). For controlling total protein amounts, as well as levels of TDP-43 and pTDP-43 in the inocula, all treatment medium were submitted to dot blotting with antibodies against actin, TDP-43 and pTDP-43.

\section{High content imaging of neurite outgrowth}

Neuritic network development was followed by phase contrast ${ }^{39}$. The signal was acquired using an Incucyte S3 platform (Essen Biosciences) with a 20x objective. White light was used for phase contrast, green and red leds for fluorescence. Neurite outgrowth was computed from both the phase contrast (time lapse) and fluorescence images (endpoint) using the Neurotrack software (Essen Biosciences). At 4 DIV, cultures were then challenged with the four concentrations of SarkoSpin pellets or medium for untreated wells. At 6 days post inoculations, effects of the treatments were evaluated by live cell staining with Calcein-AM to visualize the viable neurons and their processes.

\section{Randomization}

Data collection was randomized as control / disease patient samples were assigned randomly to experimental procedures. We also ensured that all different sample groups were included in each experiment to exclude any experiment / experimenter biases. Similarly, in all SarkoSpin, mass spectrometry, ultracentrifugation, immunolabelling and microscopy procedures, all TDP-43 proteinopathies samples were randomized with control samples. 


\section{Blinding}

Data collection and analysis were not performed blind to the conditions of the experiments, as they did not include any subjective measurement or incomplete data collection.

\section{Statistical analysis}

No statistical methods were used to pre-determine sample sizes but we used the maximum number of samples that were available to us. When stated, normality of the data sets was verified with ShapiroWilk normality test and equality of variances with an $\mathrm{F}$ test. Otherwise, data distribution was assumed to be normal but this was not formally tested. We used t tests for two-sample comparisons and oneway or two-way ANOVA followed by Tuckey's multiple-comparison tests for more than two samples. Statistical analyses were performed with GraphPad Prism 7 software.

\section{Data availability}

Mass spectrometry raw files were uploaded to the ProteomeXchange Consortium via the PRIDE partner repository. Dataset identifier: PXD007873. The data that support the findings of this study are available from the corresponding author upon request.

\section{Compliance with ethical regulations for animal procedures}

All experiments using mice and mouse embryos were carried out in accordance with the European Communities Council Directive 2010/63/EU on the care of animals used in scientific research and approval by the ethics committee of the University of Bordeaux (C2A50). 


\section{References:}

46. Leek, J.T., Johnson, W.E., Parker, H.S., Jaffe, A.E. \& Storey, J.D. The sva package for removing batch effects and other unwanted variation in high-throughput experiments. Bioinformatics 28, 882-883 (2012).

47. Ling, S.C., et al. ALS-associated mutations in TDP-43 increase its stability and promote TDP-43 complexes with FUS/TLS. Proc Natl Acad Sci U S A 107, 13318-13323 (2010). 
a

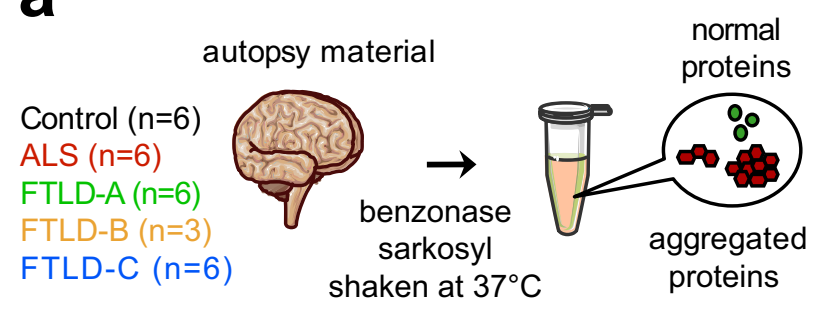

FTLD-B $(n=3)$

FTLD-C $(n=6)$

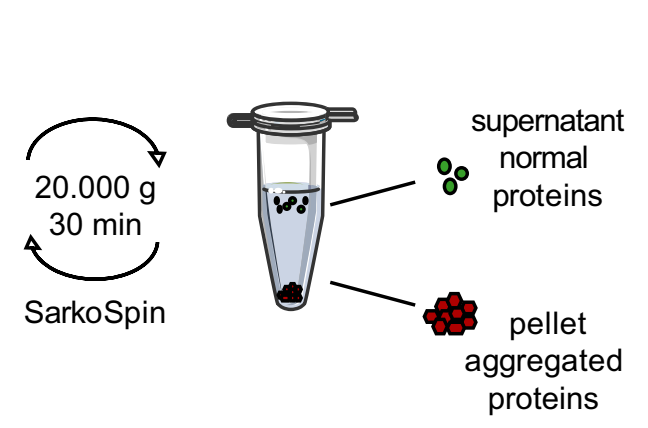

C

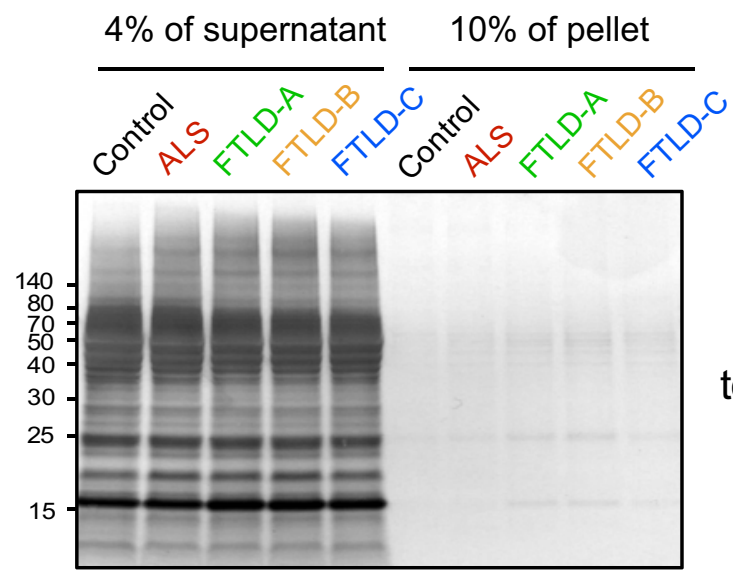

e

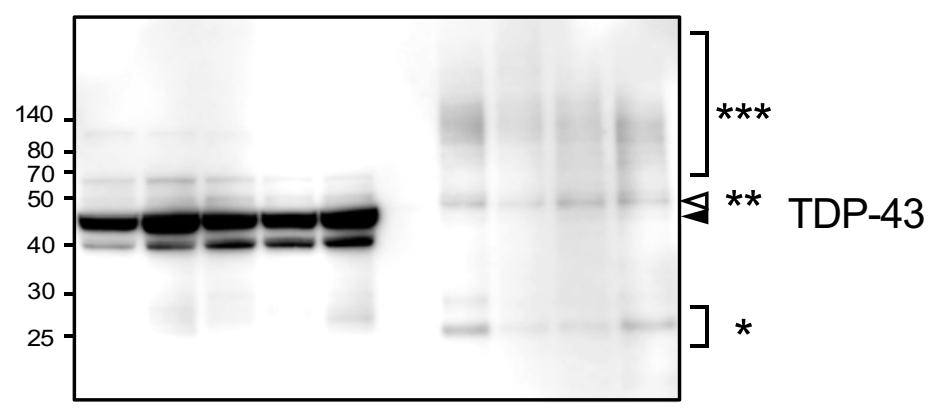

g

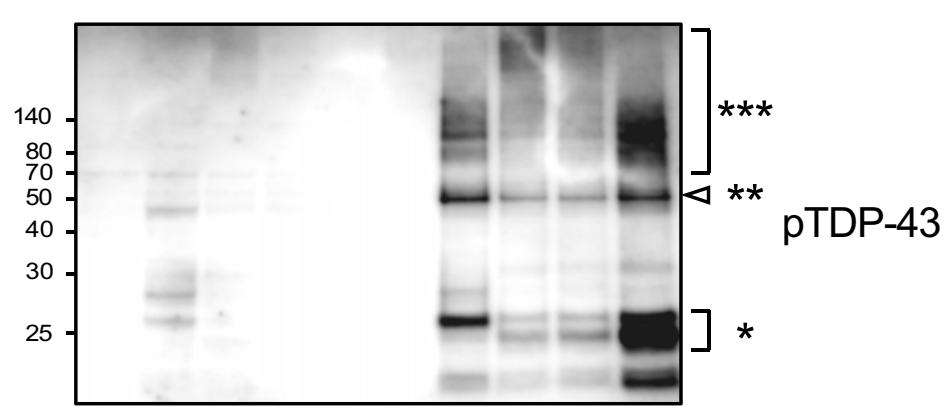

i

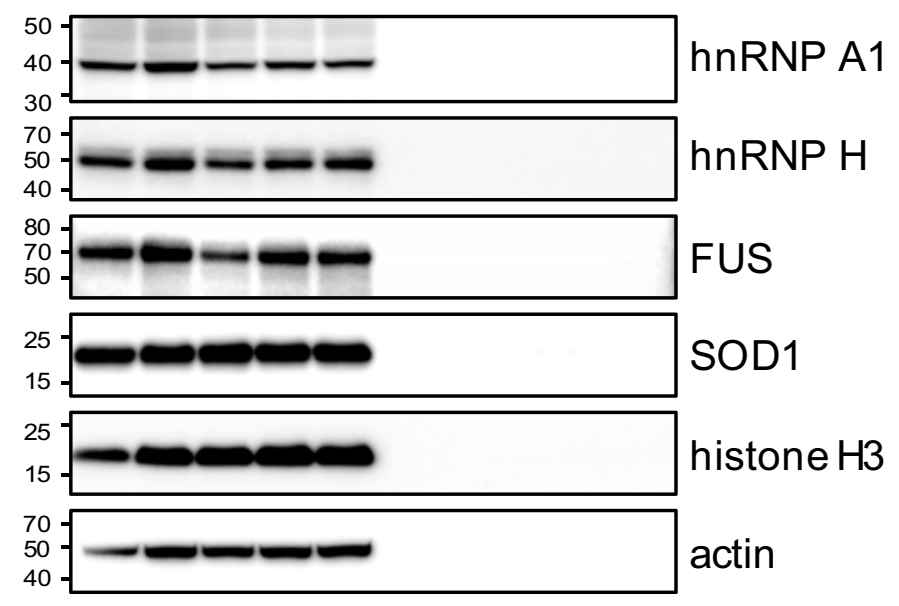

d

b

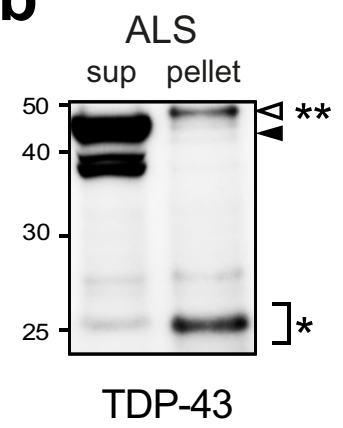

total protein

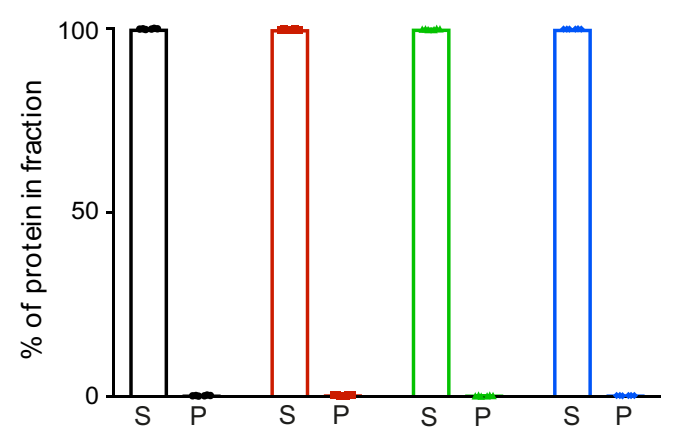

f

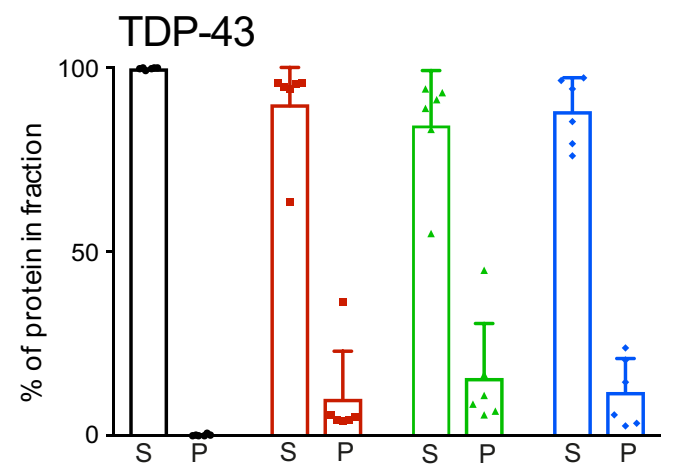

h

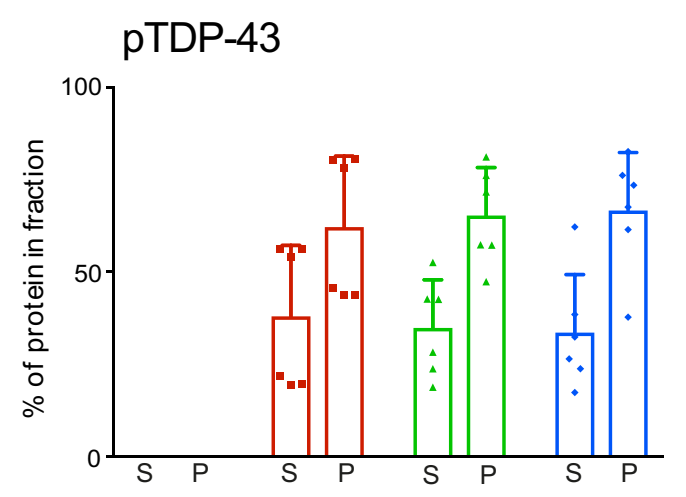

hnRNP A1

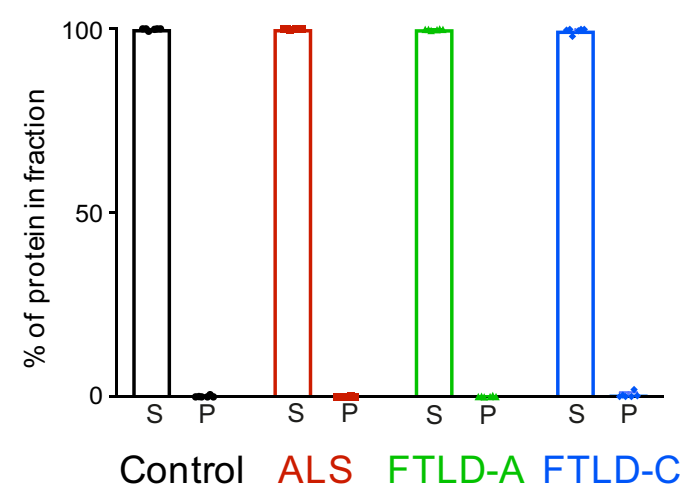




\section{a}

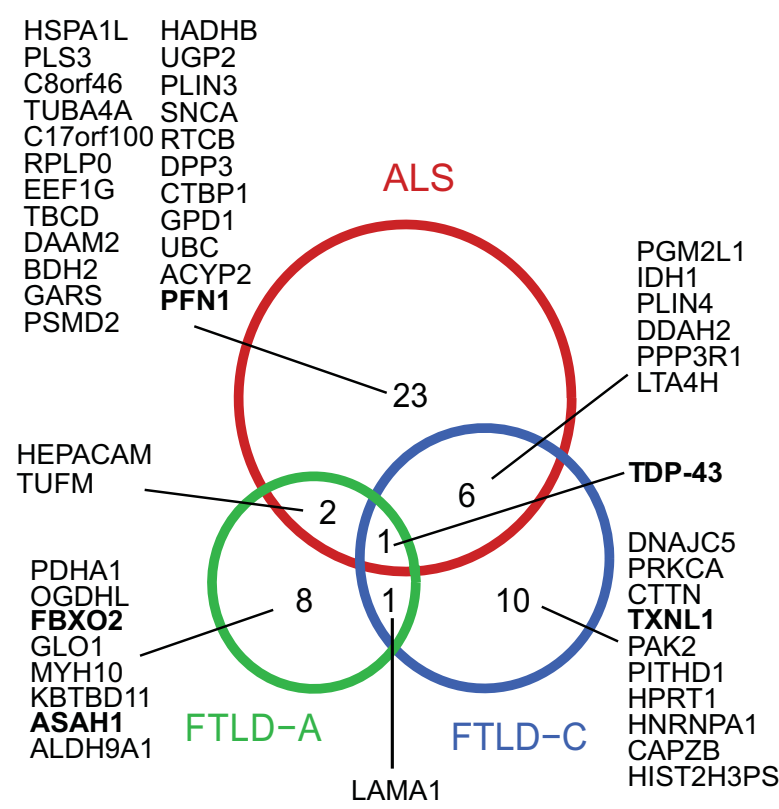

d

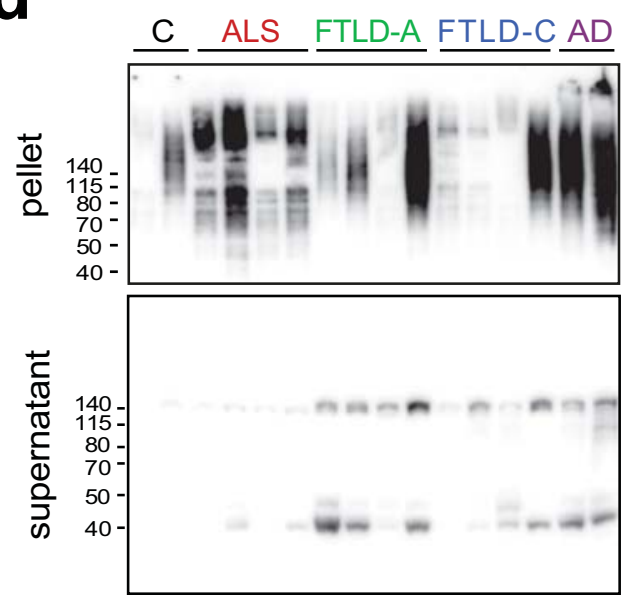

Ubiquitin

h

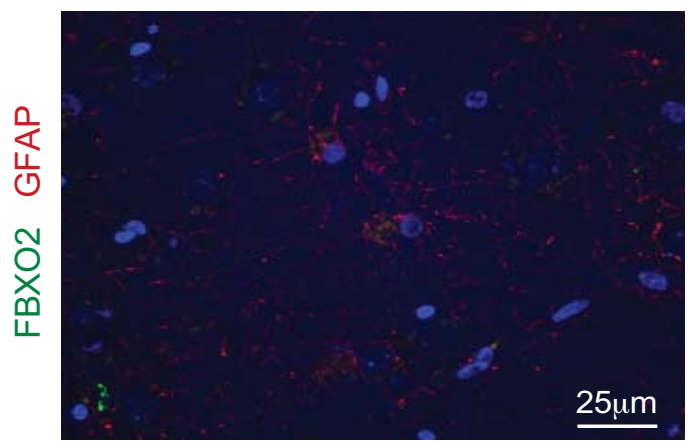

e

g b

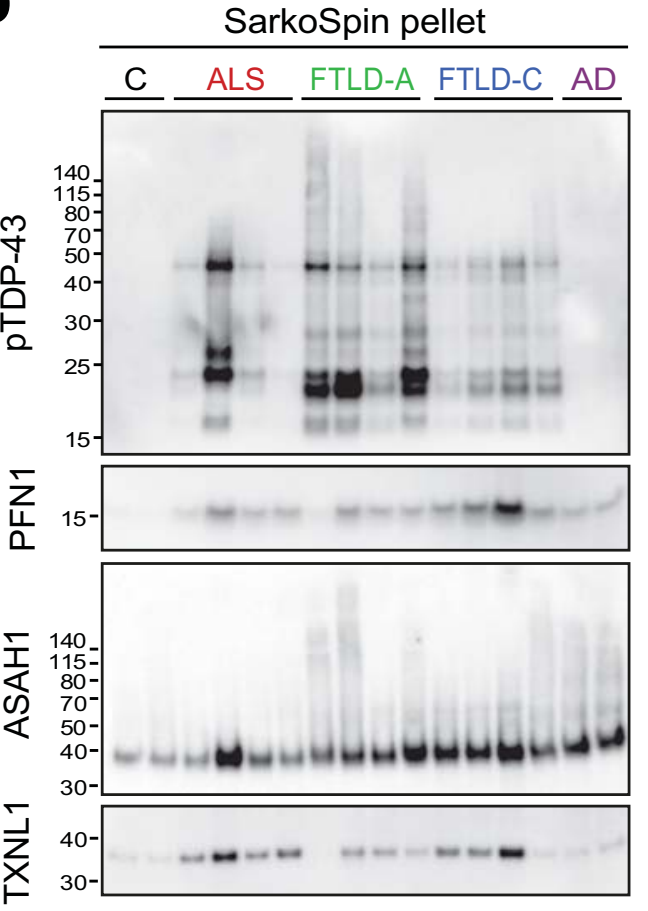

C $\rightarrow$ ALS $\bullet$ FTLD-A $\multimap$ FTLD-C
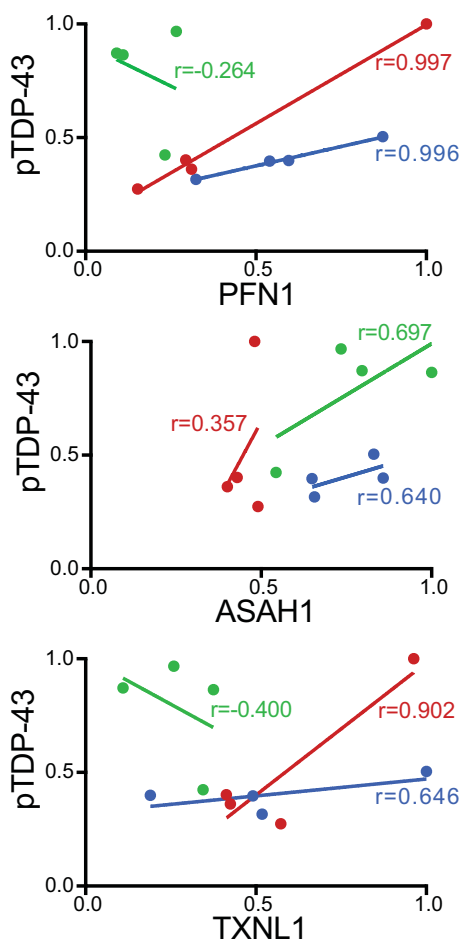

f
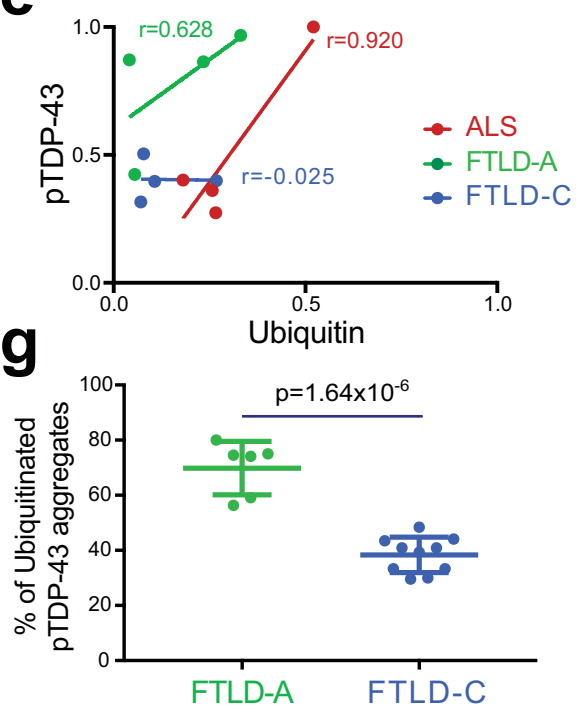

FTLD-A

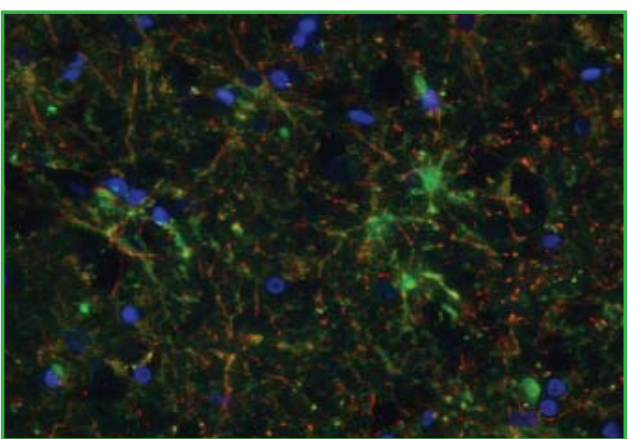

pTDP-43 Ubiquitin
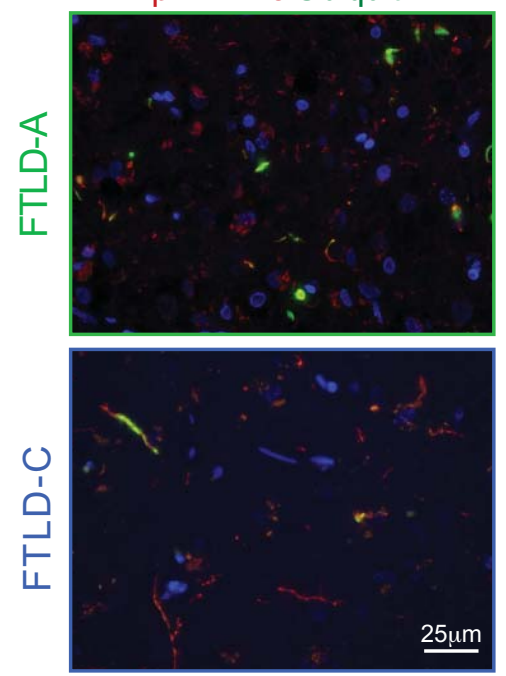

FTLD-C

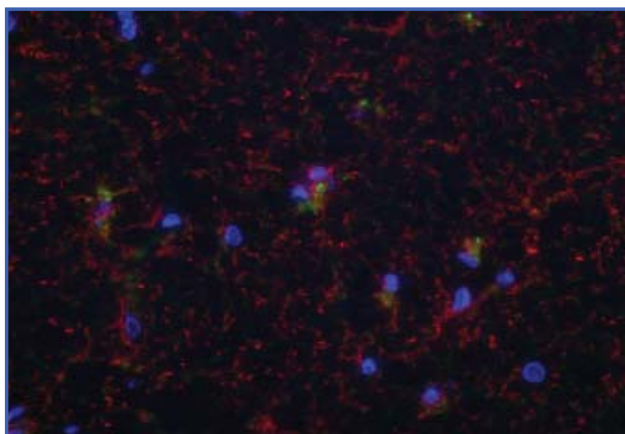




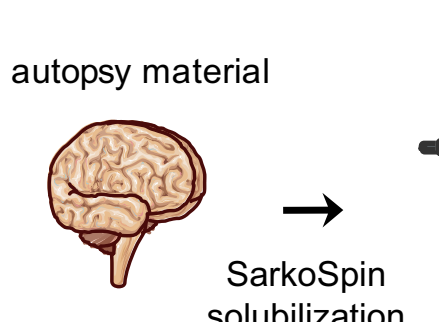

solubilization

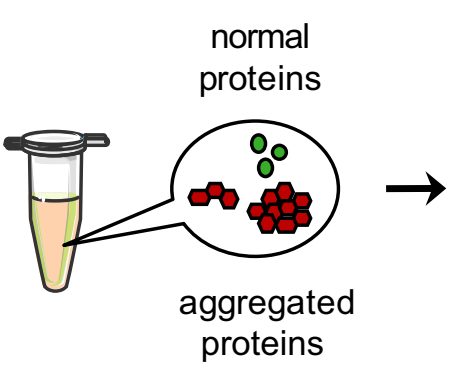

b

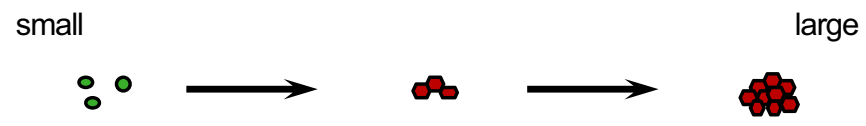

$\begin{array}{lllllllllllllllll}\text { fractions } & 1 & 2 & 3 & 4 & 5 & 6 & 7 & 8 & 9 & 10 & 11 & 12 & 13 & 14 & 15 & 16\end{array}$
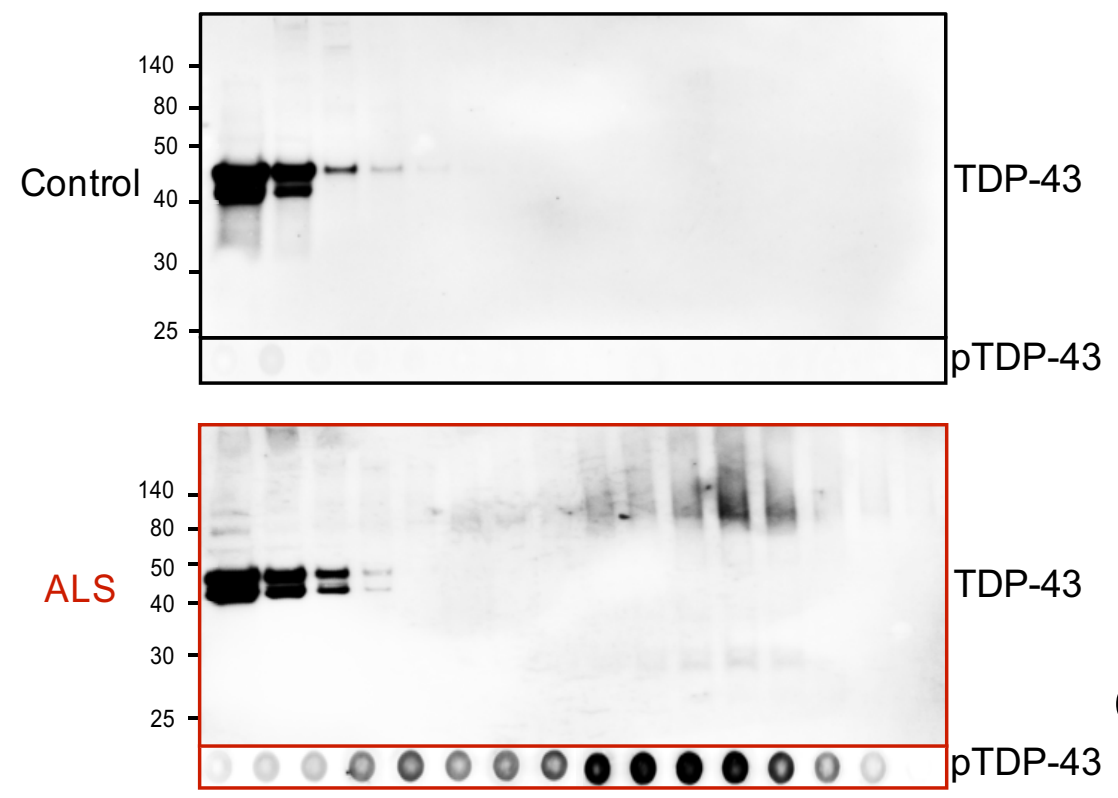

TDP-43

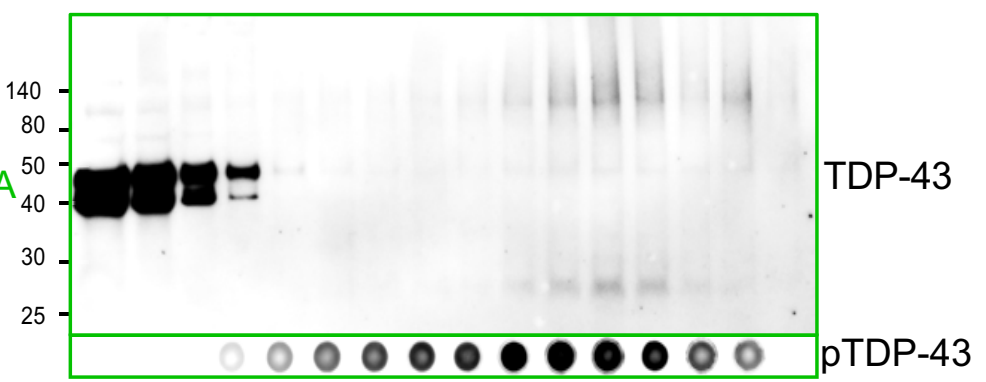

FTLD-

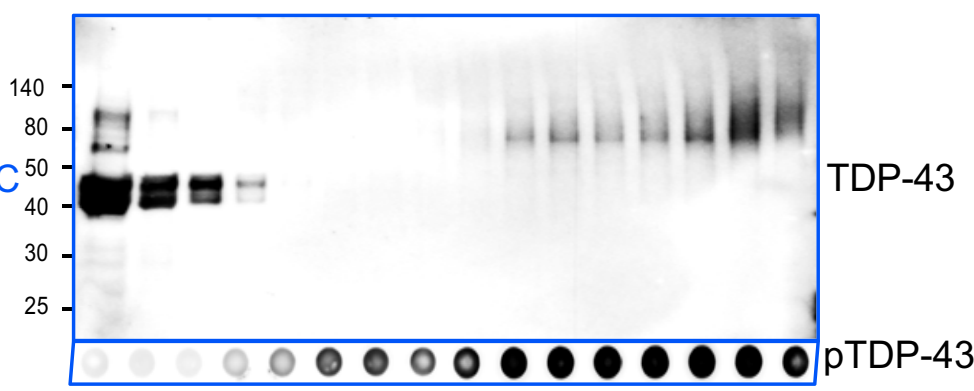

FTLD-C ${ }_{40}^{50}-{ }^{2}=$

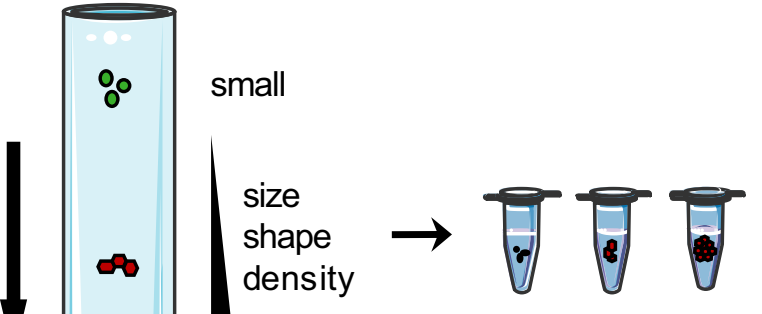

fractionation of the entities based on their sedimentation

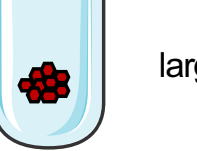

C

TDP-43

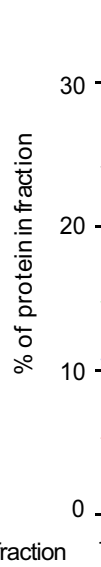

Control $n=6$

ALS $n=4$

FTLD-A $n=11$

FTLD-C $n=9$

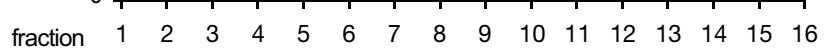

\section{Control / ALS \\ Control / FTLD-A \\ Control / FTLD-C \\ FTLD-A / ALS \\ FTLD-A / FTLD-C \\ ALS / FTLD-C}

d

pTDP-43
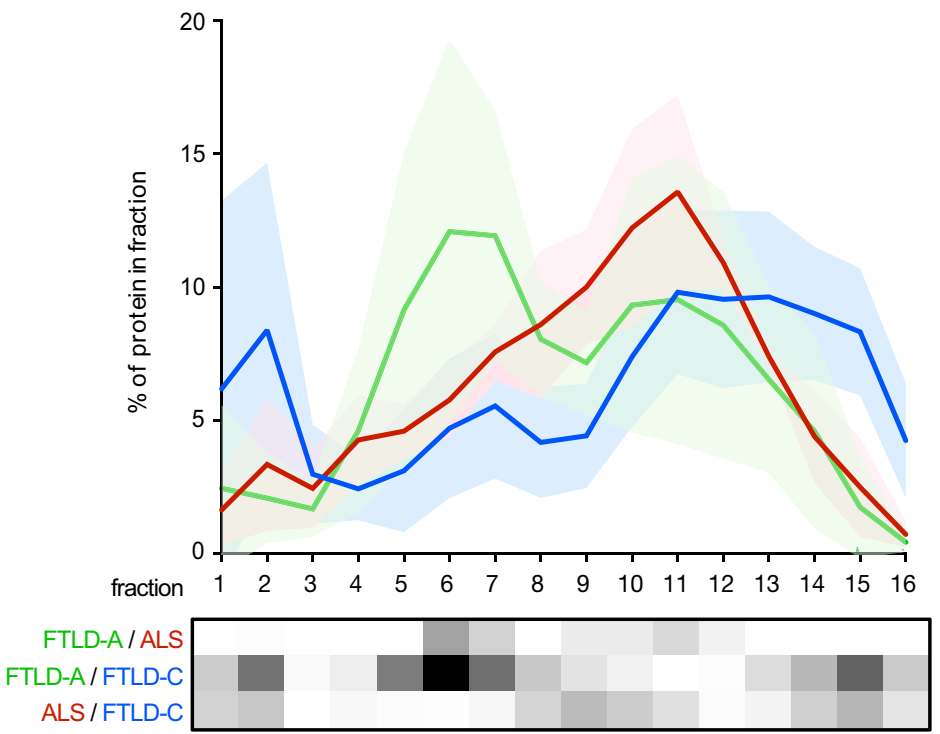

- $\log 10$ (P Value)

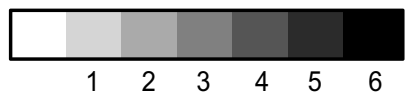



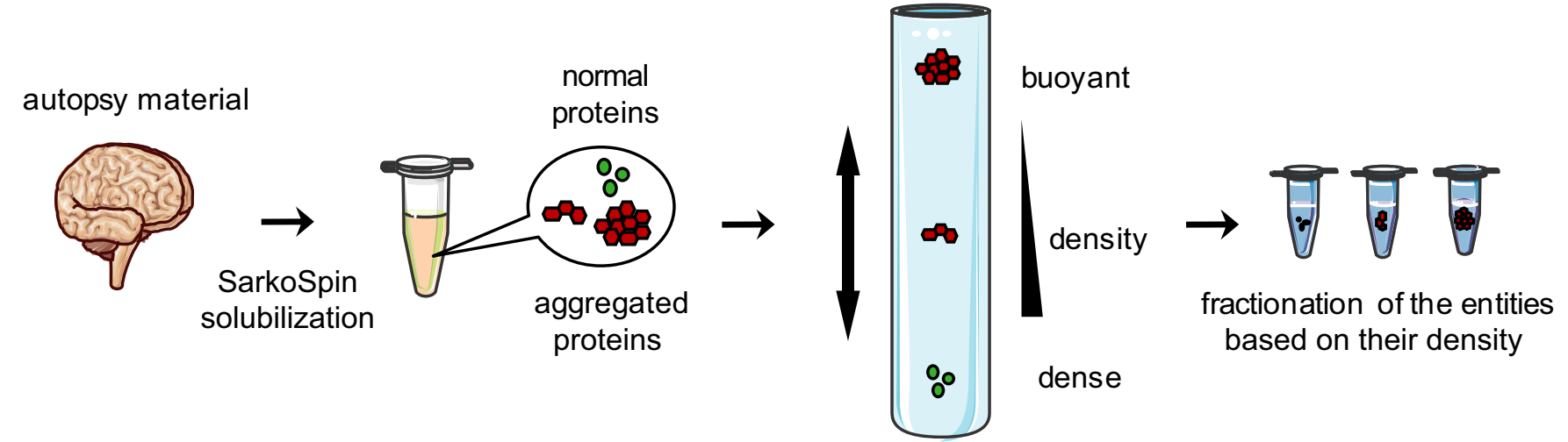

b

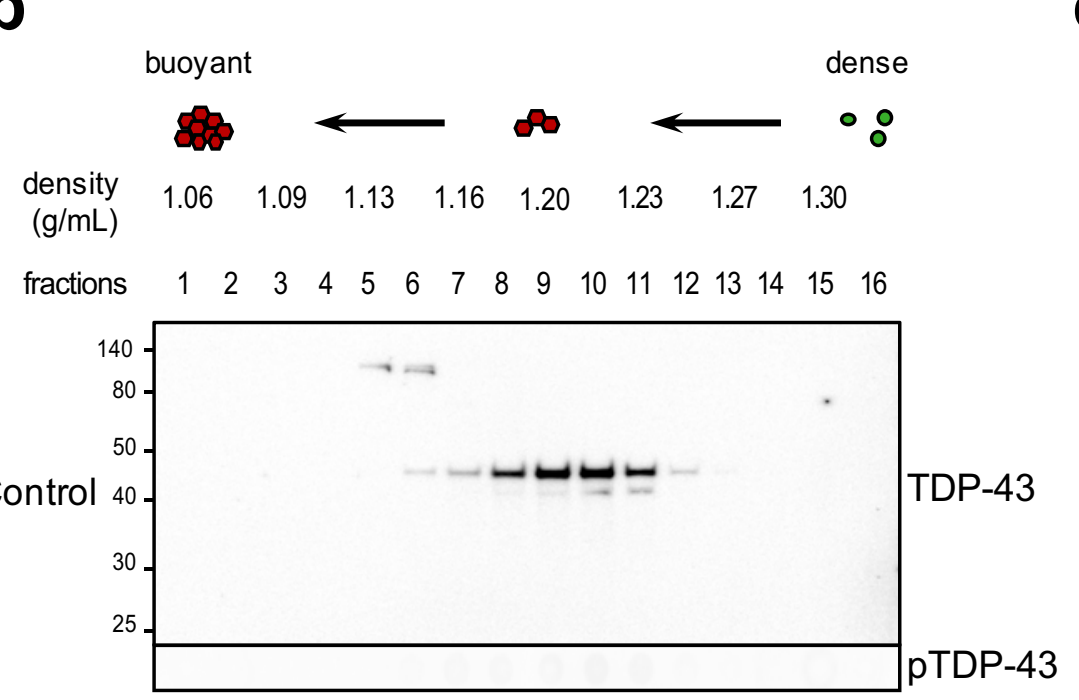

C

TDP-43

Control $n=6$

ALS $n=4$

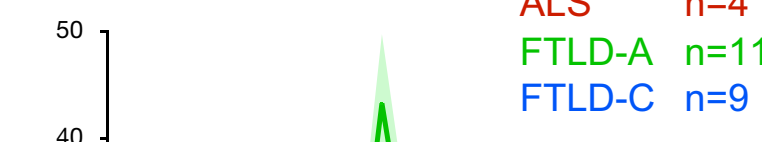

ALS

$\begin{array}{lllllllllllllllll}\text { fraction } & 1 & 2 & 3 & 4 & 5 & 6 & 7 & 8 & 9 & 10 & 11 & 12 & 13 & 14 & 15 & 16\end{array}$
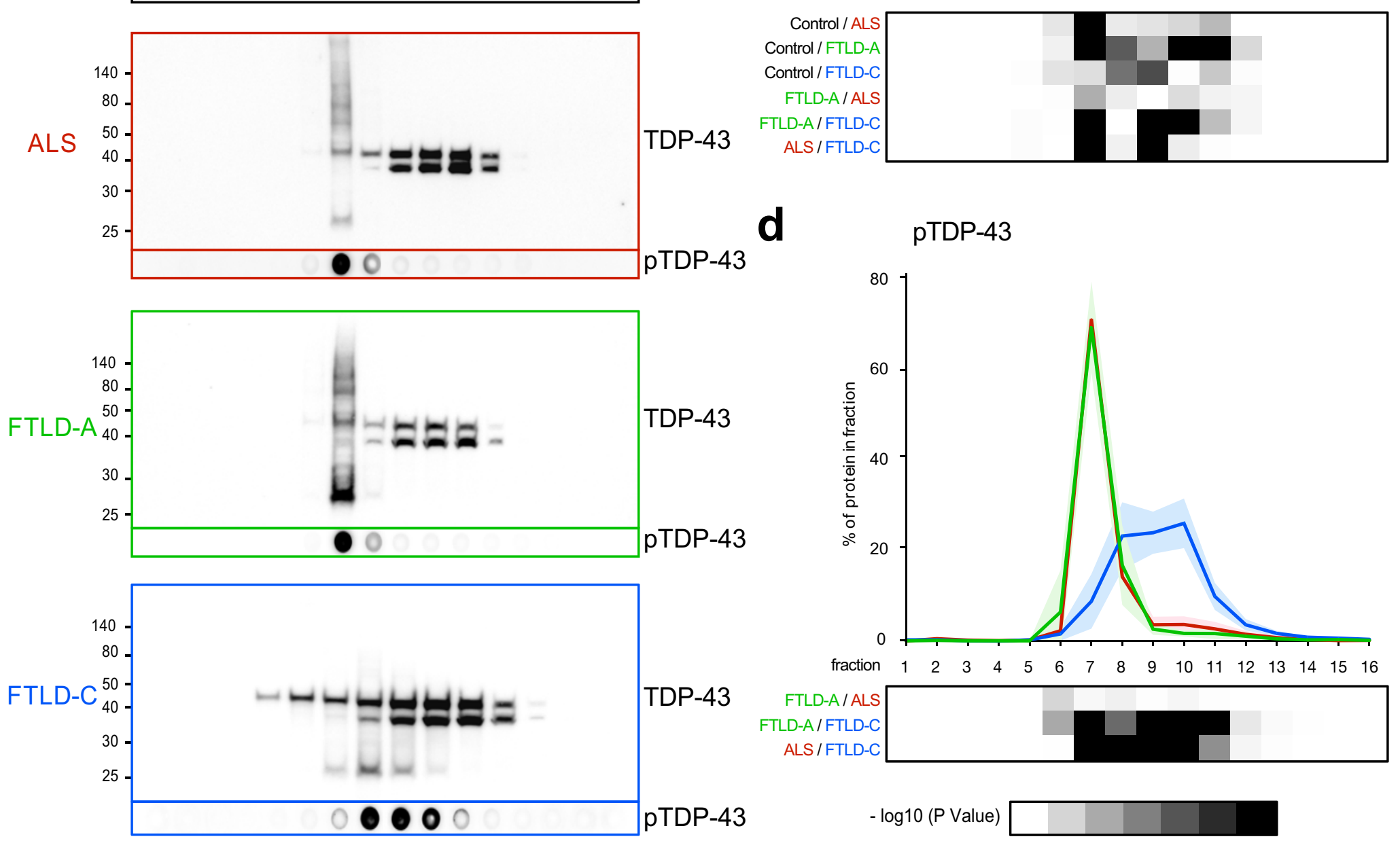

d

pTDP-43
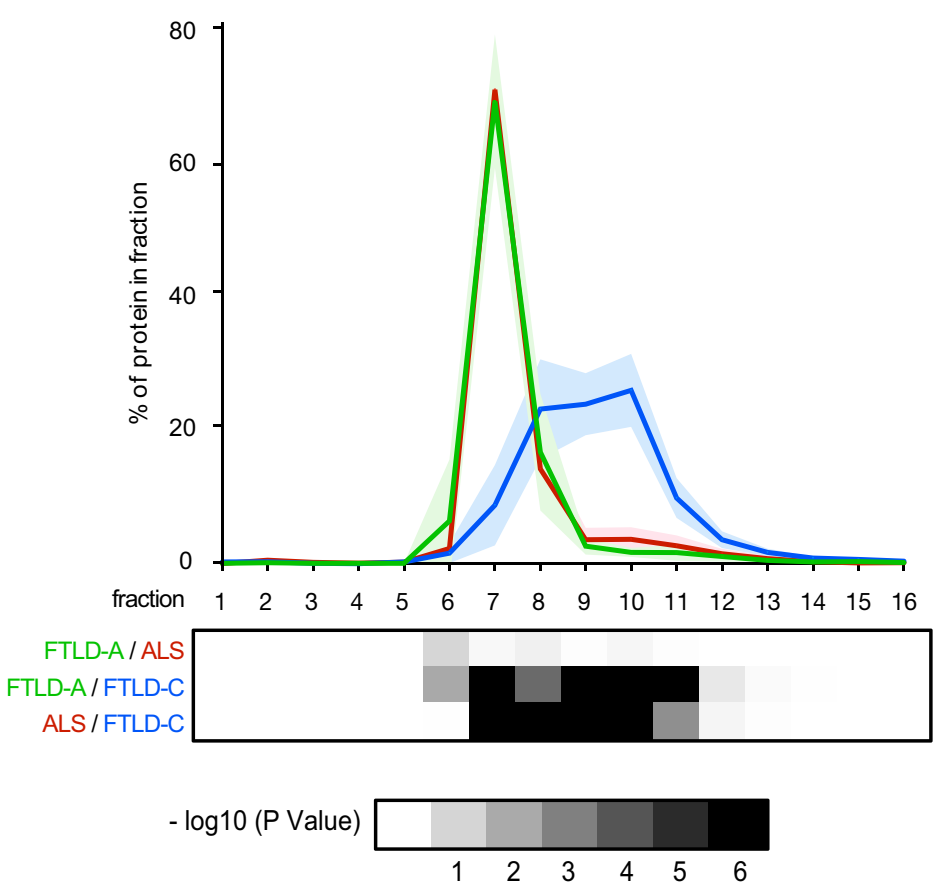
a

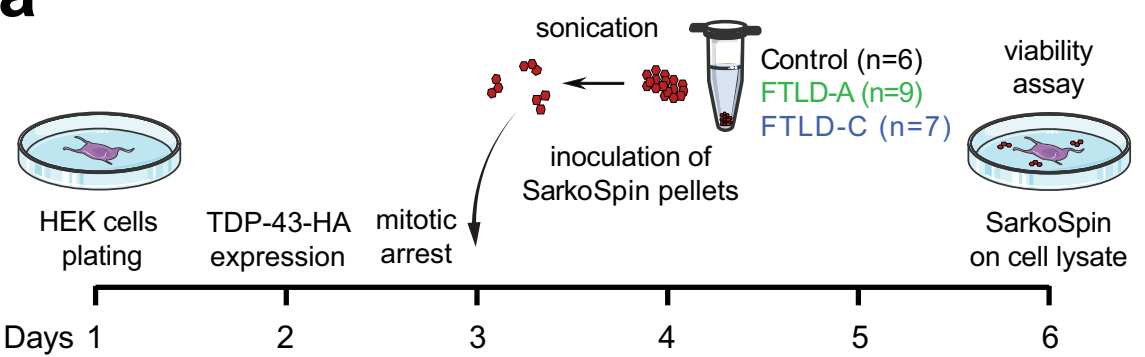

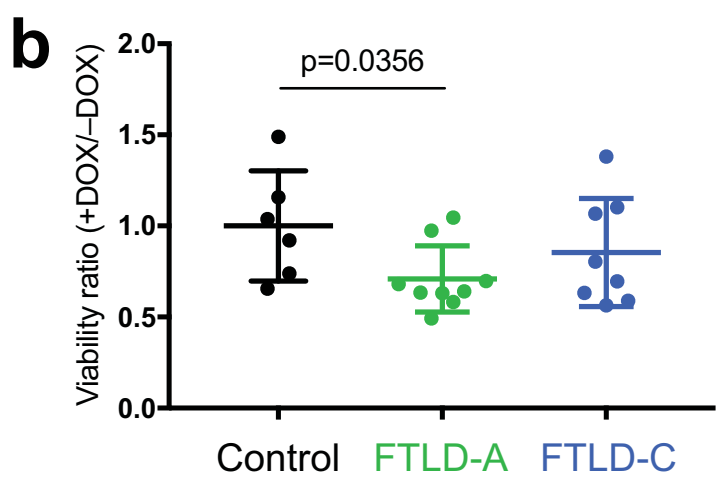

C

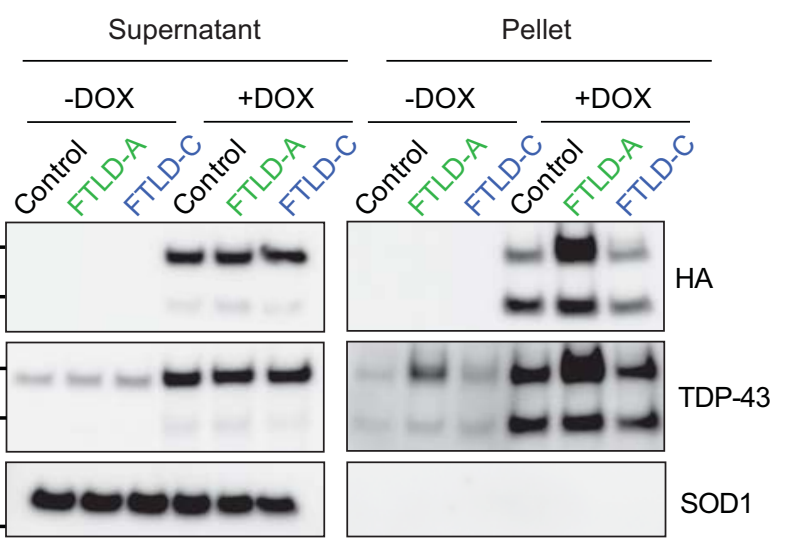

d

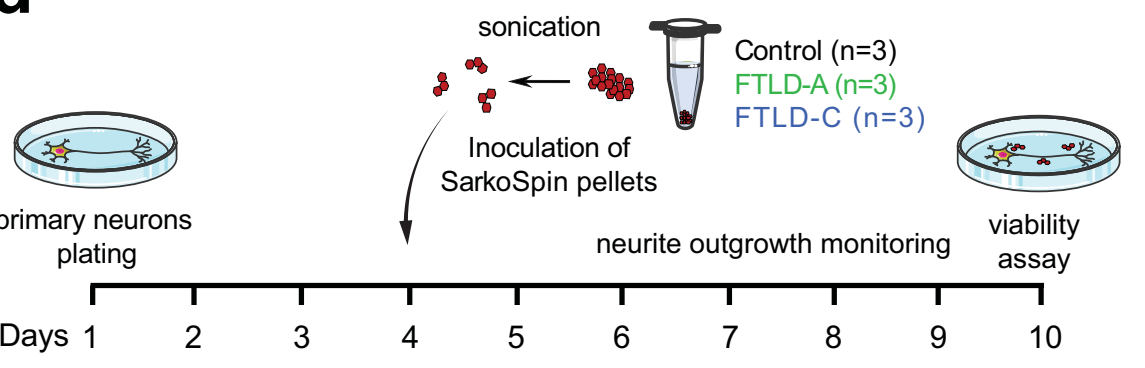

e

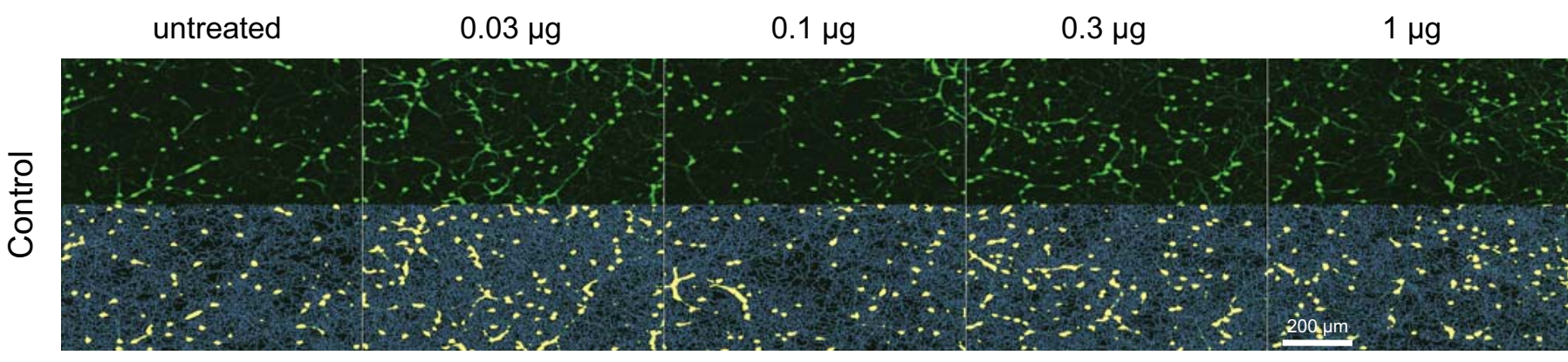

live

cells

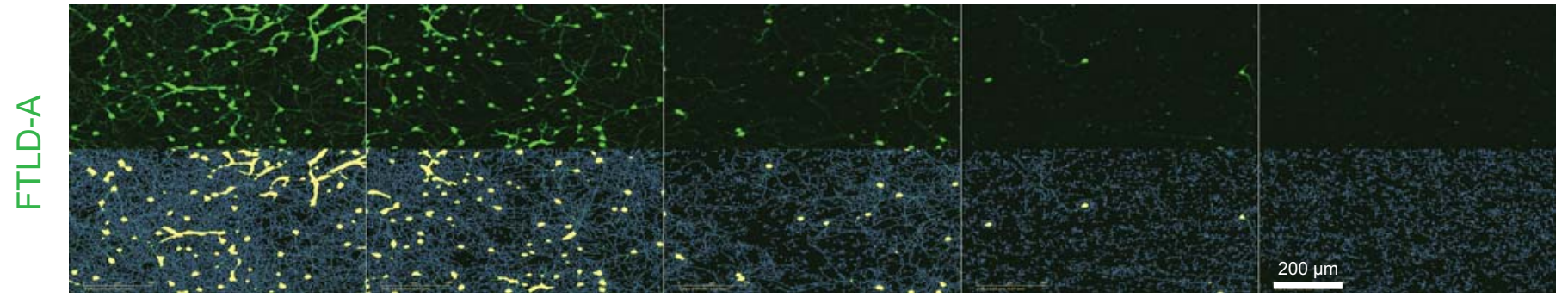

live

cells

cell

bodies

neurites

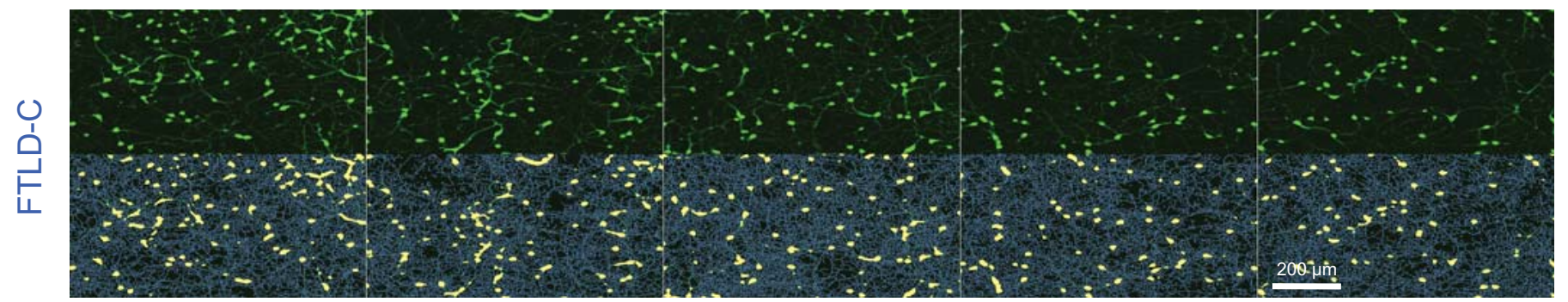

live

cells

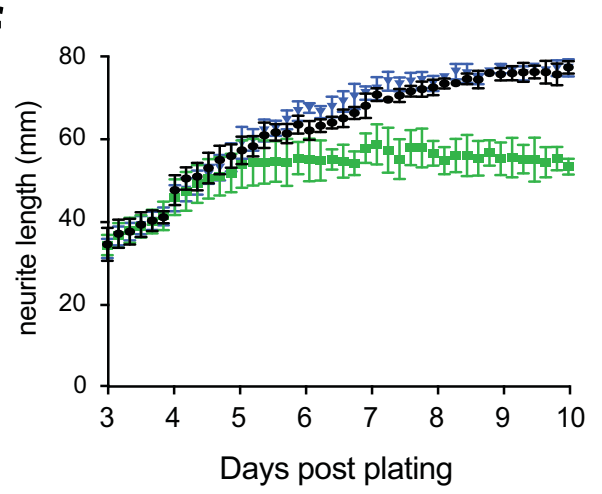

g

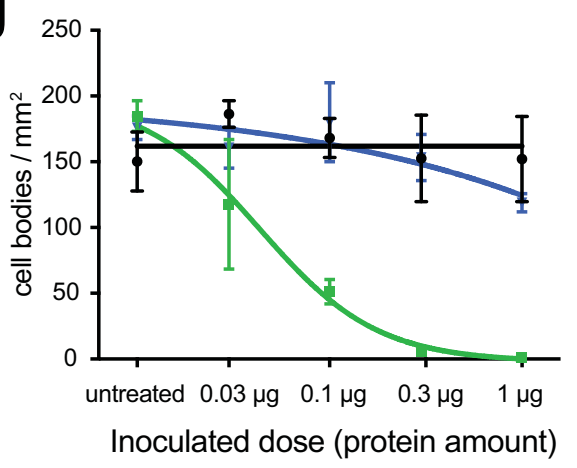

h

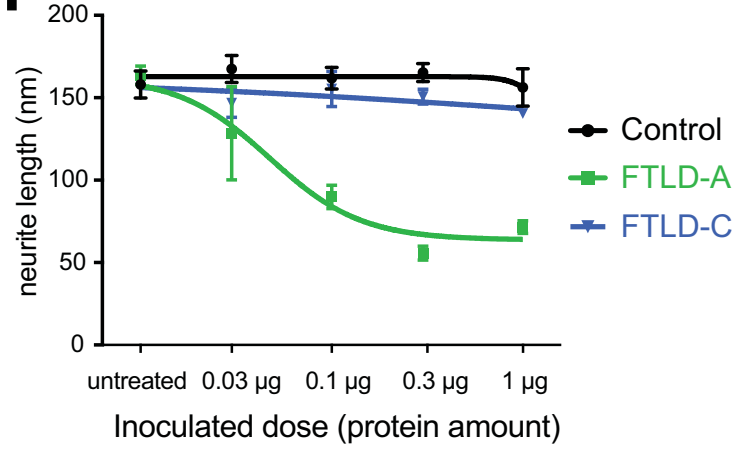


a

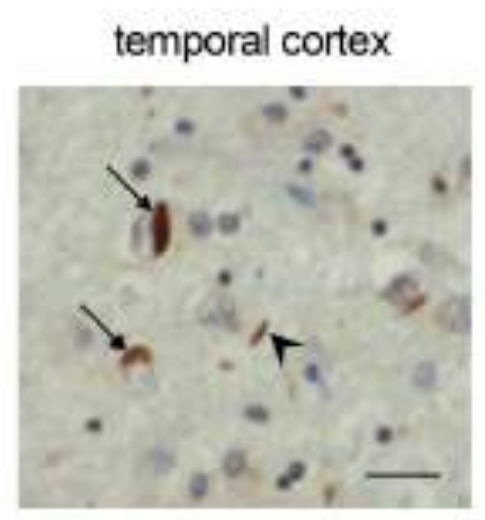

FTLD-A

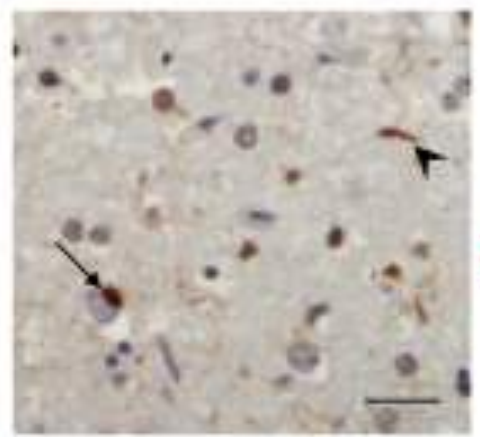

FTLD-A
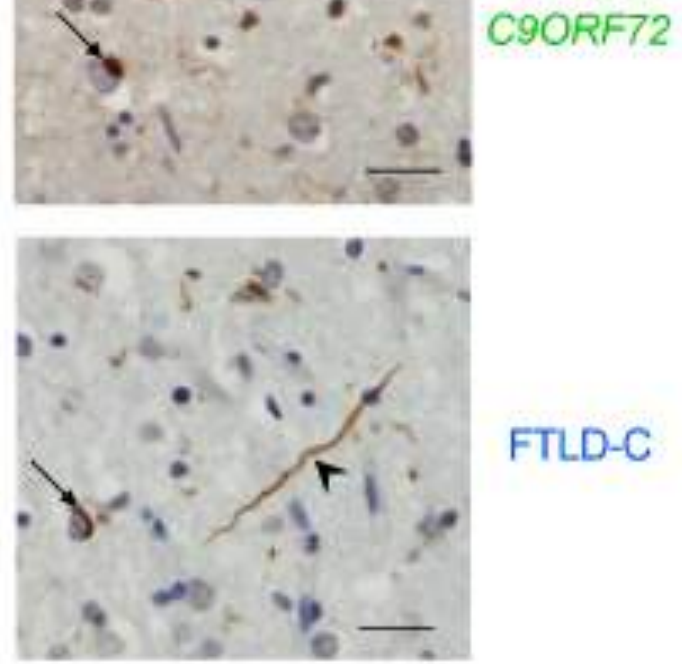

pTDP-43 b

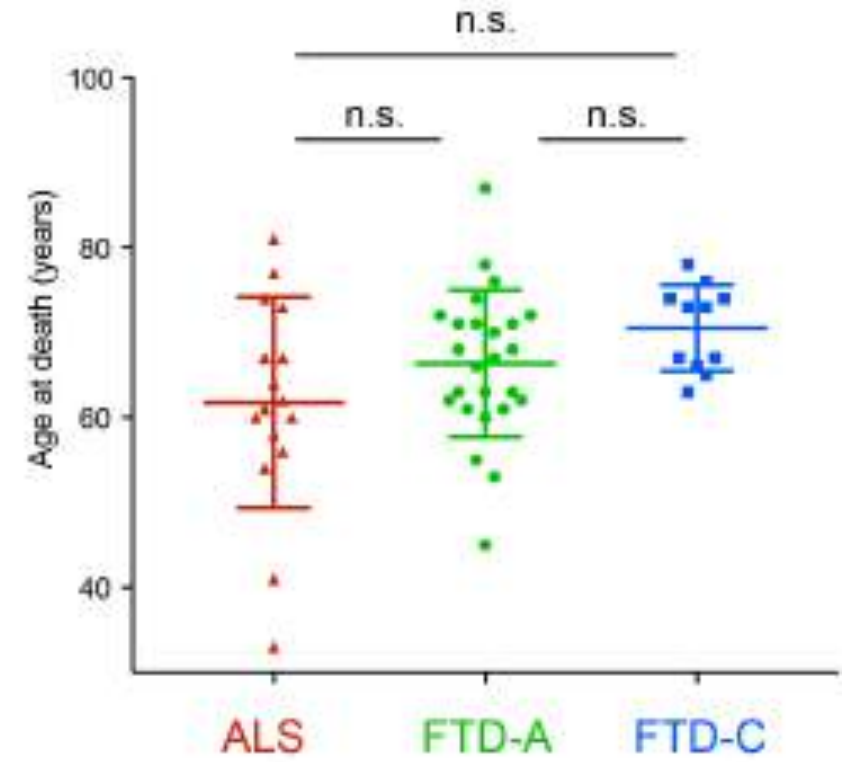

C

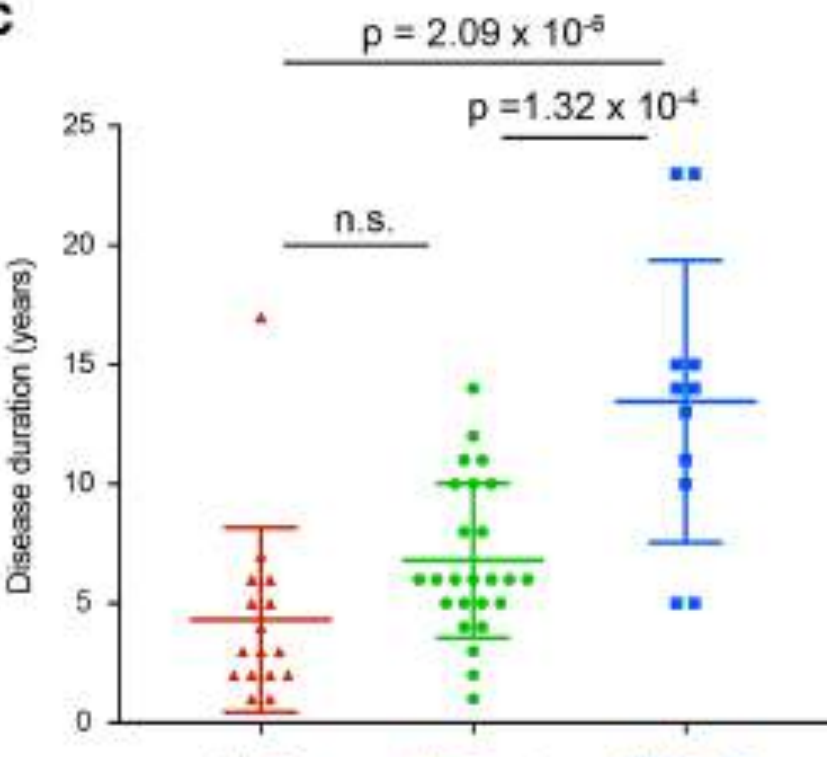

ALS FTD-A FTD-C

\section{Supplementary Figure 1}

Characteristics of disease subtypes and correlation to disease duration.

a. Phosphorylated TDP-43 immunohistochemical staining of temporal cortex from FTLD-TDP subtypes, highlighting differences in their pathological hallmarks. Neuronal cytoplasmic inclusions (NCl) (arrows) and short dystrophic neurites (DN) (arrowhead) were observed in FTLD-TDP-A without (top panel) or with (middle panel) C9ORF72 repeat expansions. In FTLD-TDP-C, long twisted neurites were observed (lower panel, arrowhead). Scale bar $25 \mu \mathrm{m}$. This experiment was repeated two independent times with similar results. b-c. Age at death (b) and disease duration (c) of patients from the different disease groups used in the study (error bars show means and SD). FTLD-TDP-C patients showed significantly longer disease duration. $n=16,25$ and 11 individual patients for ALS, FTLD-TDP-A and FTLD-TDP-C respectively. One-way ANOVAs followed by Tuckey's multiple-comparison tests gave the indicated $p$ values. 


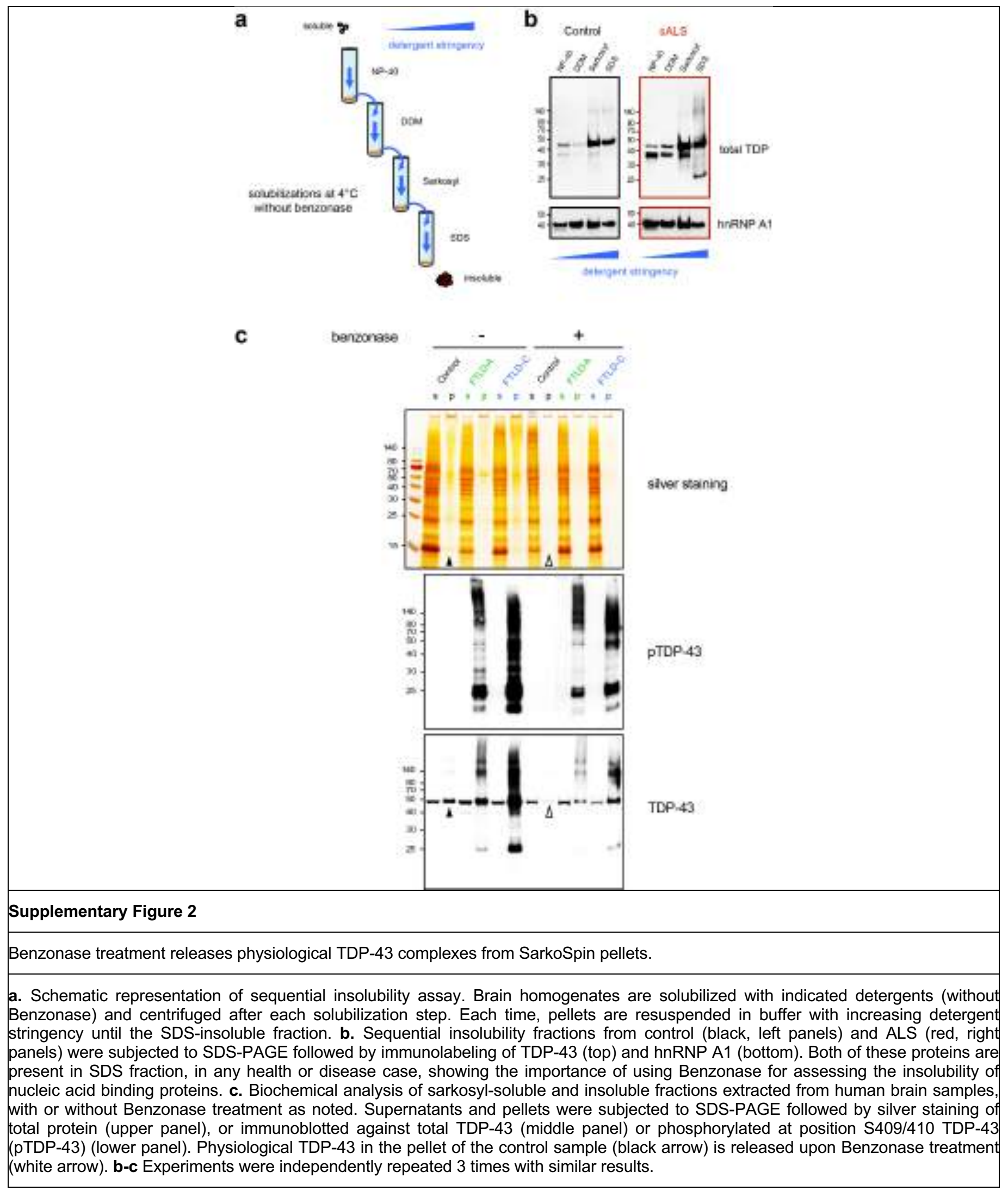




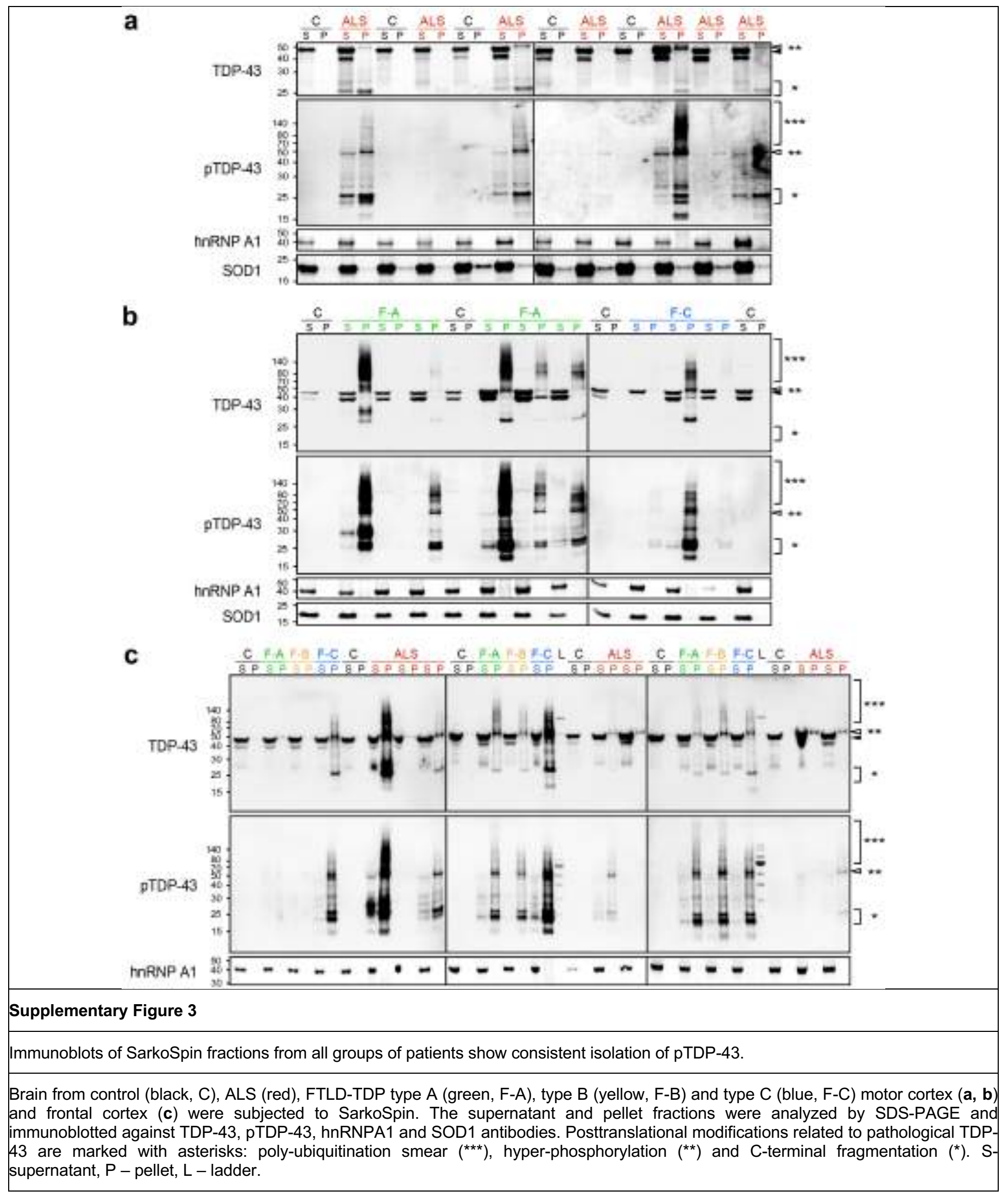




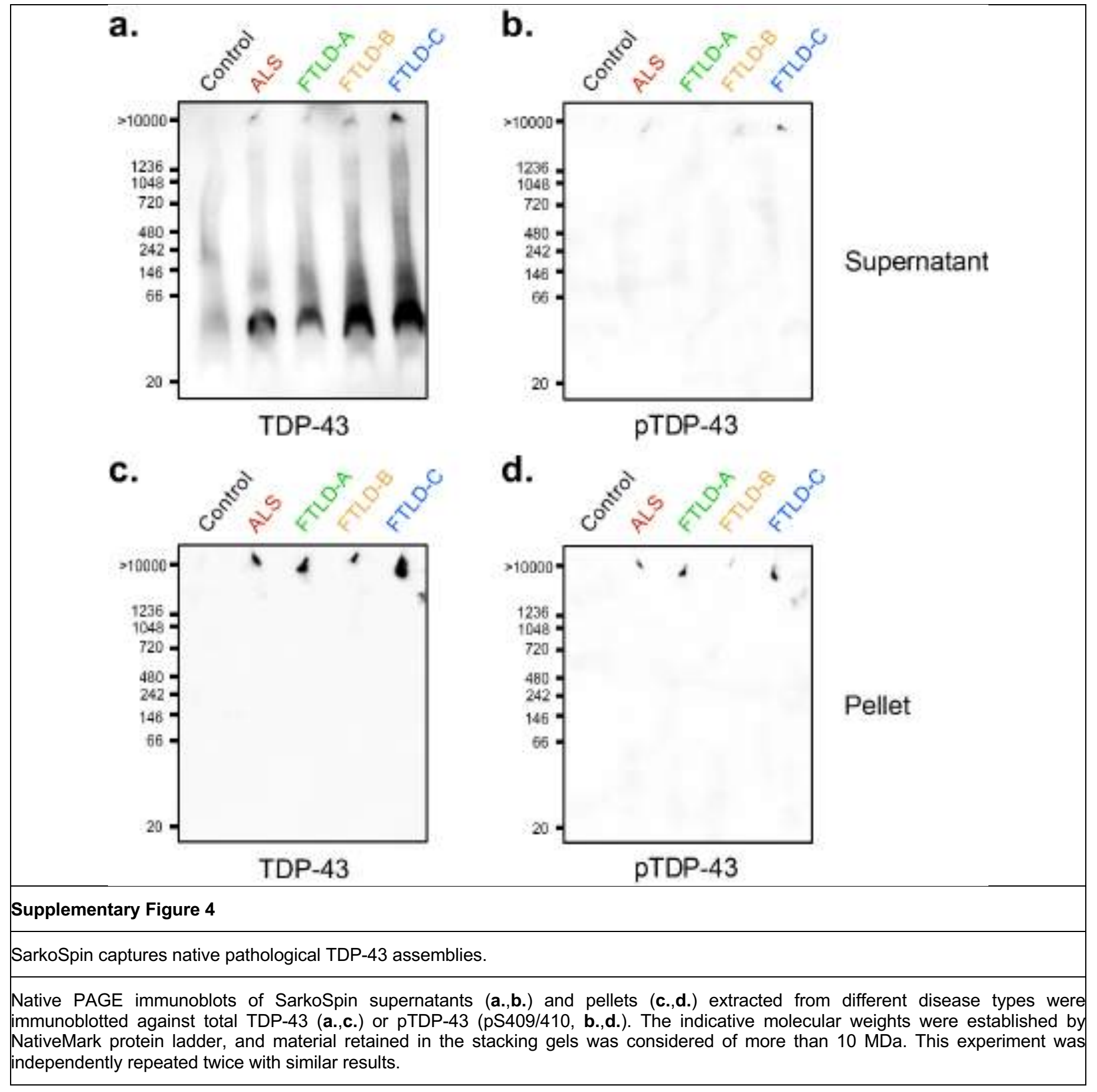




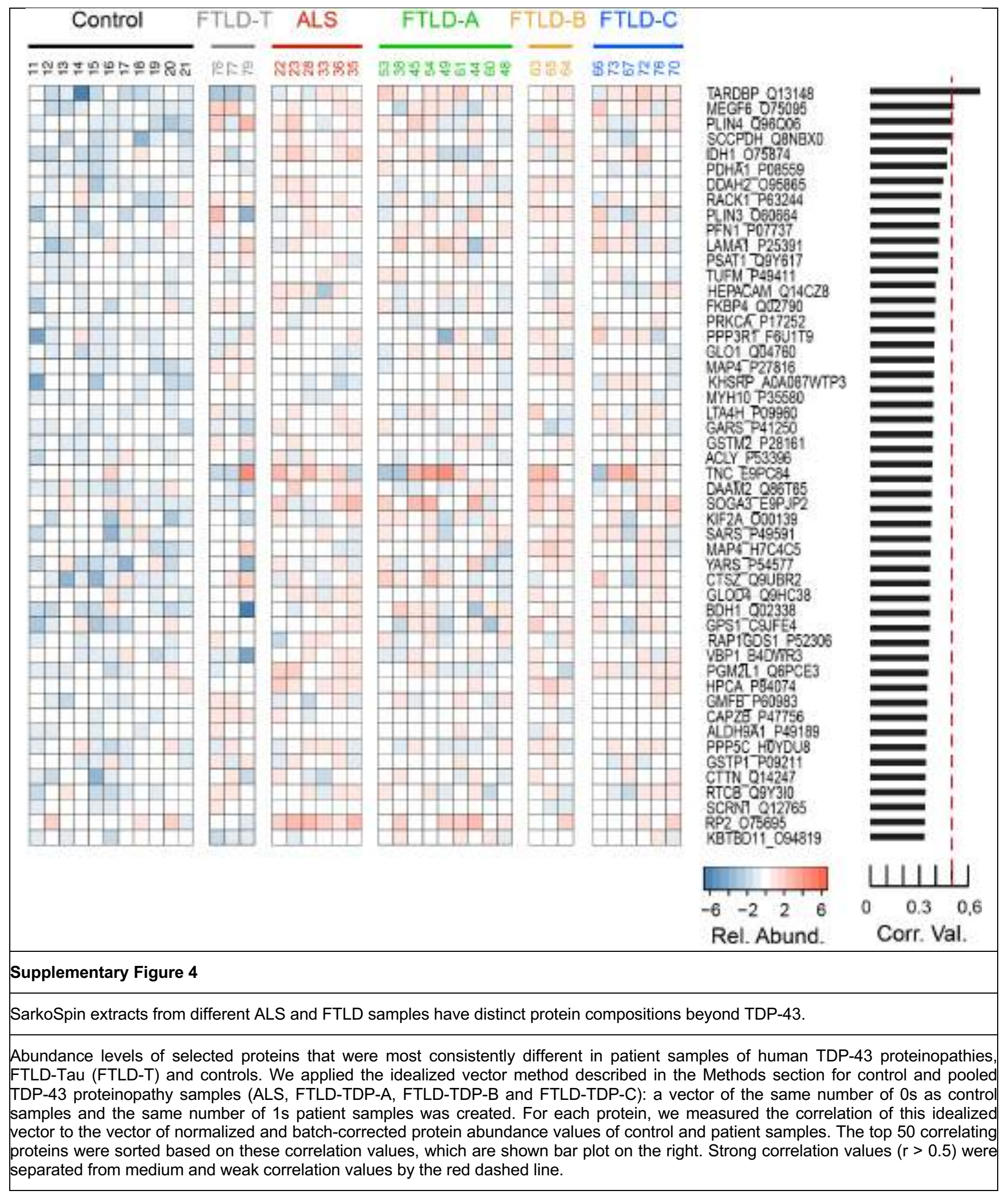


HSPA1L P34931

PLSS AOAAOANSCO

DH1 075874

CBCr'P6 OSTAGS

PGIVL 1 OSPCE3

TAROBP O 13149

LTA4H P09980

TUEA4A CQIJOS

C17ort100 D3OTMS

C17OR PO P05388

RPLPO PJ5388
DDAH2 095665

DDAH2 O95866
EEF1G P266541

TUFM P494:

TBCD CogTw9

Dám Ceste

Danile cosito

$\mathrm{OH} 2 \mathrm{C} 9 \mathrm{BU}$

GARS P41250

PSMO2 Q13200

HADHE P5S084

- LiN4_096006

UGP2 A0A007WYS

PPP3R1 FEU1T9

HEPACAM Q14CZ8

PLIN3 OEOEG4

PLIN3 O60664
SNCA ETEPV7

SNCA EJEPV?

OPP3 DaNr33

cTep 013263

GPD1 P21695

GP.

$A C Y P 2 P 146$

ACF 12 P P 1462

SFXN3 AOADAONISA1

AOCK2 075116

ACLY P53396

AUPY3 O7Log

ispis a

USP14 A6NA

RASAL1 095294

MAP2K2 P 56507

CAANS1 ASYMT2

COPS4 099778

ARF4 P16005

ADX P35241

GSTM2 P28161

TU日 A A Q71use

TUEA1A Q71LB

CLASP2 E3IV994

109412 EapK5

SRT2 FGWCF4

NEDDE-MDP1 EgPL57
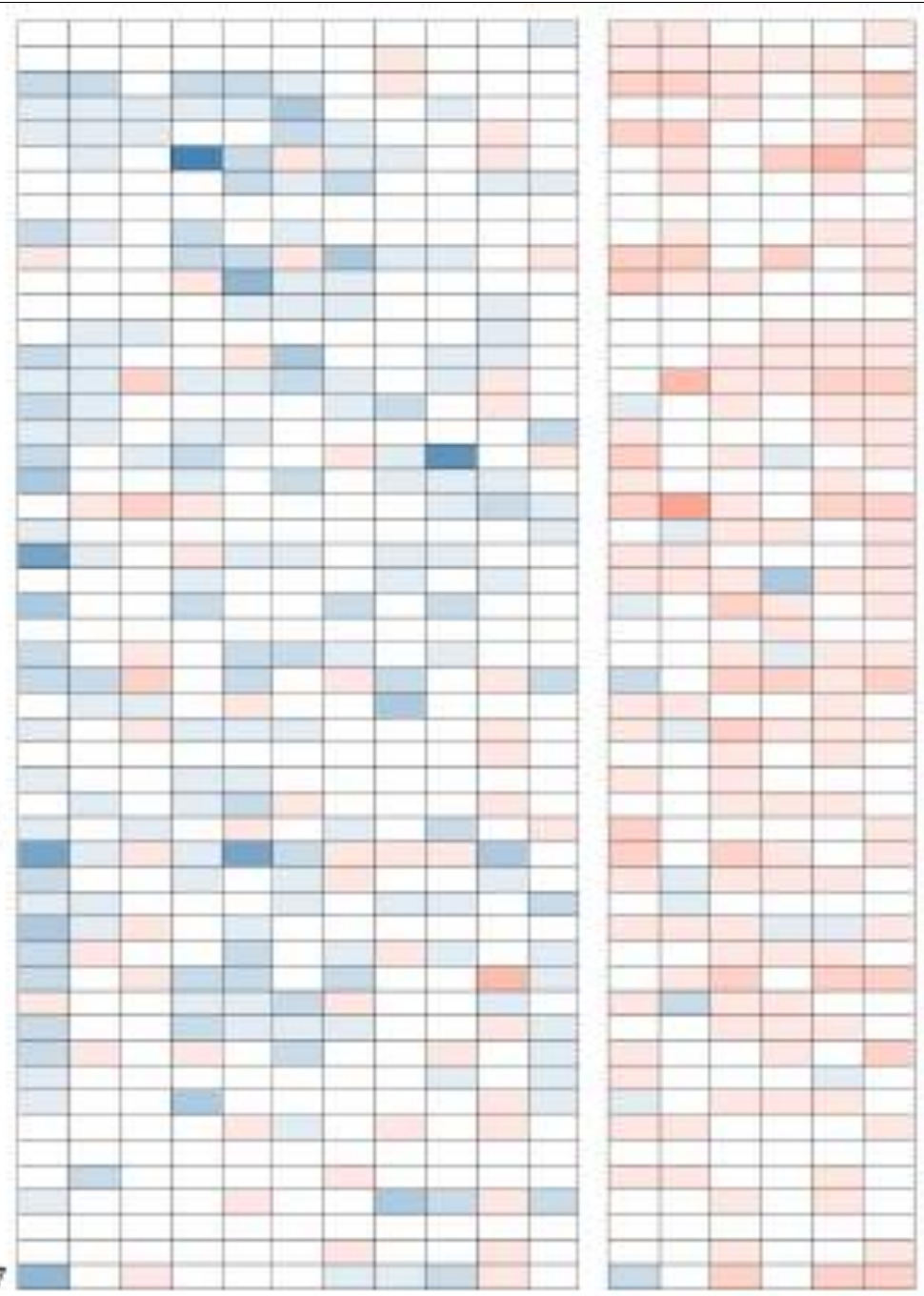

1112131415161718192021

222328333635

Control

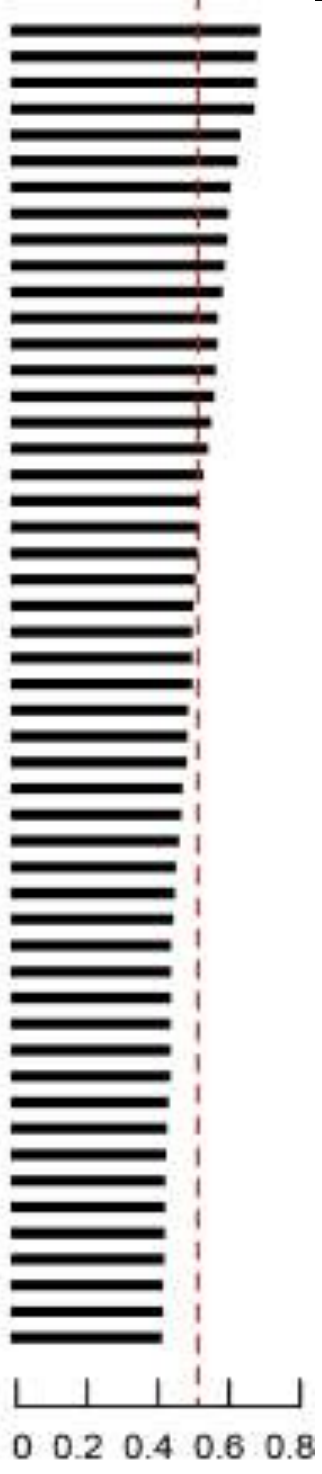

Corr. Val.

Corr. val.

Rel, Abund.

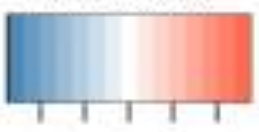

$\begin{array}{llll}-4 & 0 & 2 & 4\end{array}$

\section{Supplementary Figure 5}

Proteins enriched in SarkoSpin pellets of ALS brains.

Abundance levels of selected proteins that were most consistently different in ALS brain samples compared to controls. We applied the idealized vector method described in Methods section and Supplementary Figure 5 legend to control and ALS samples. The top 50 correlating proteins were sorted based on these correlation values, which are given as a bar plot on the right. Strong correlation values $(r>0.5)$ were separated from medium and weak correlation values by the red dashed line. 


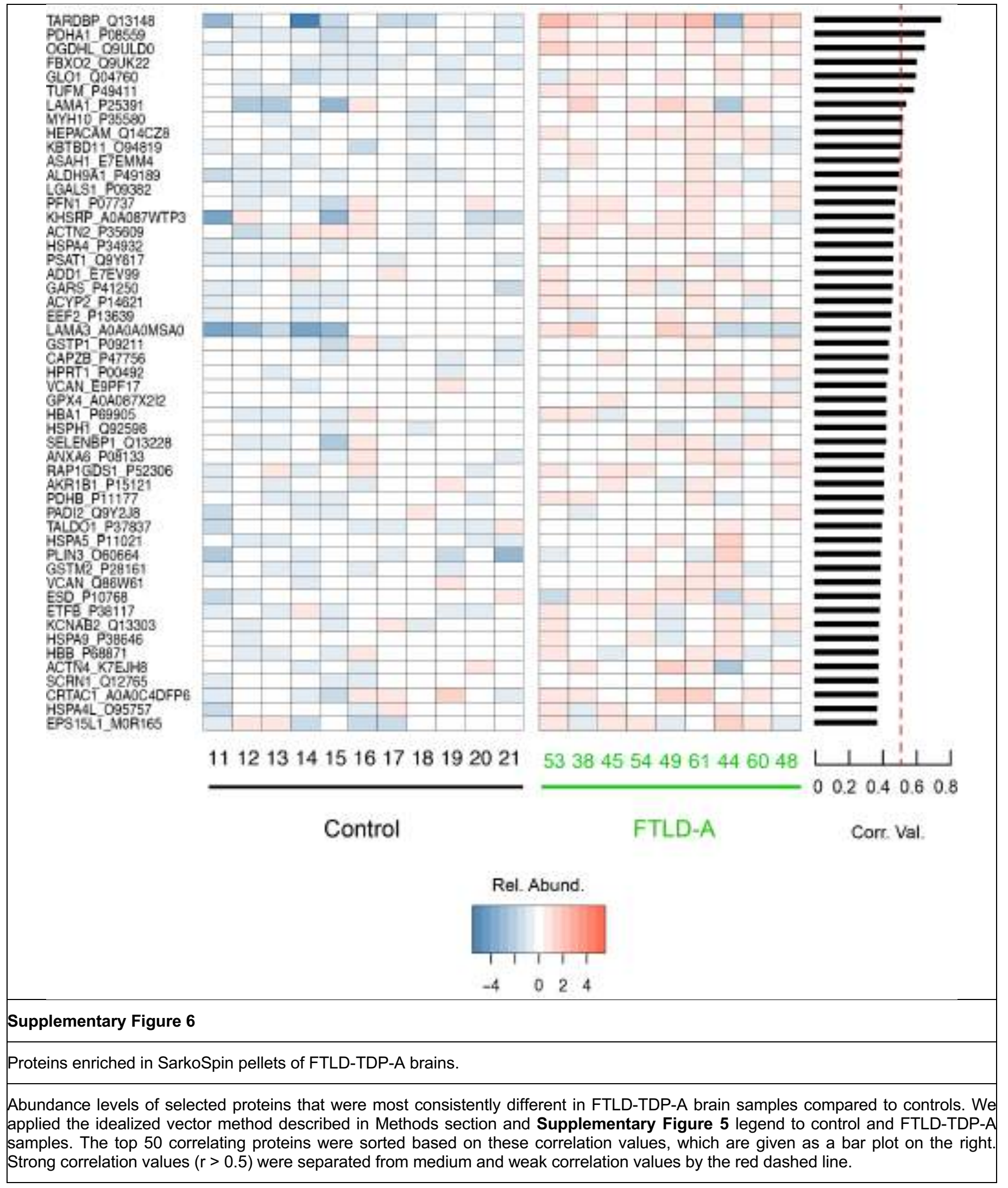




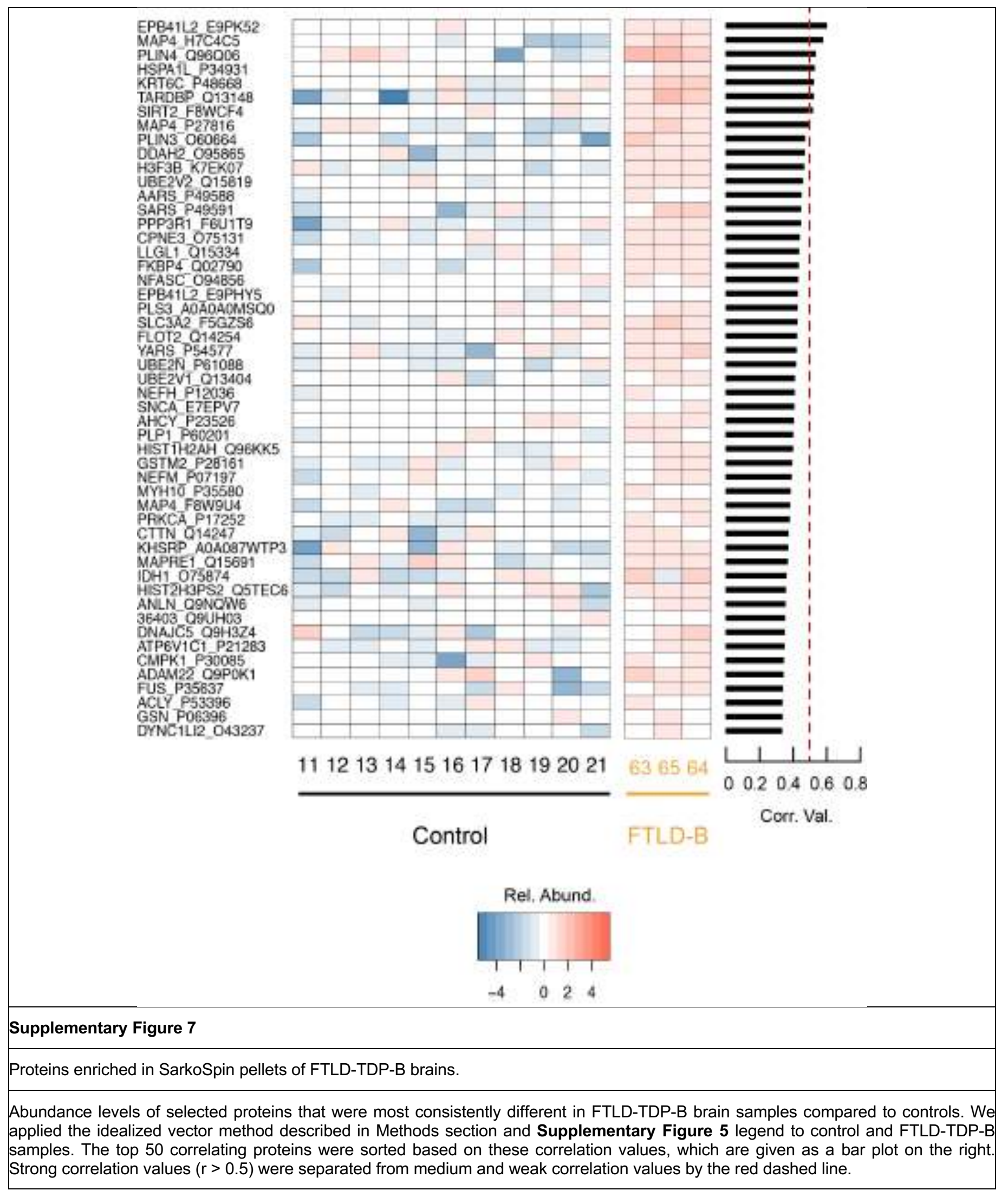




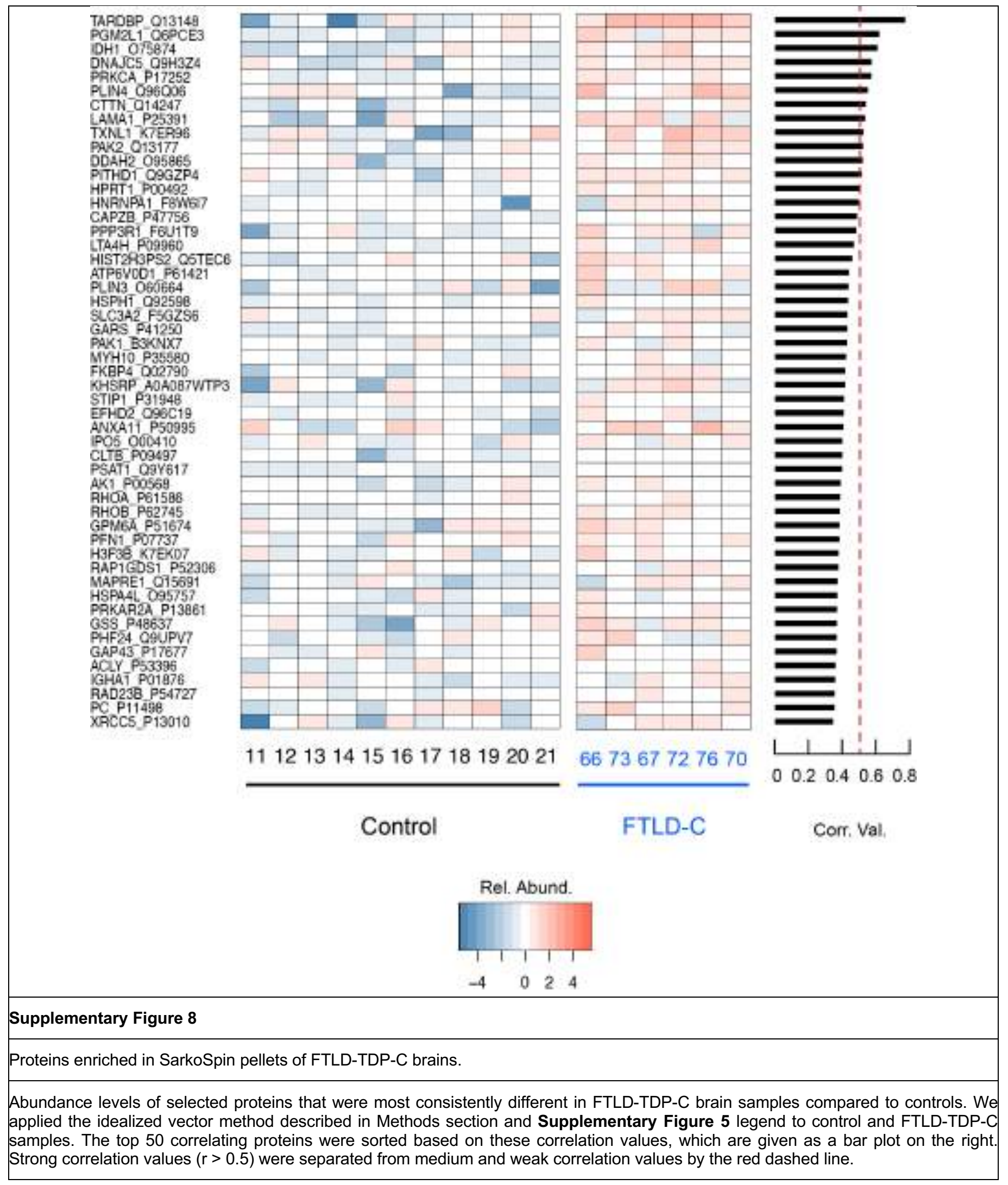




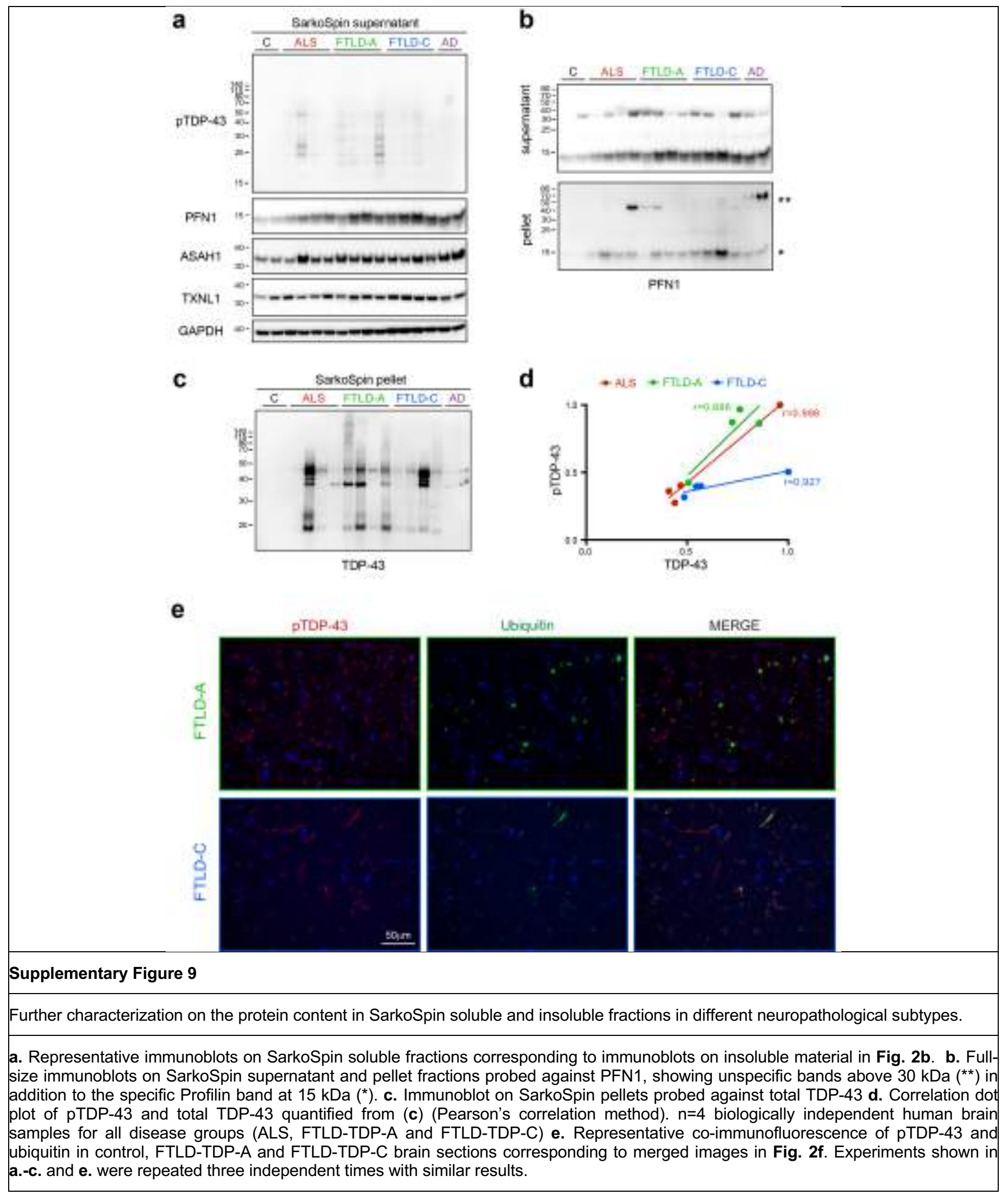




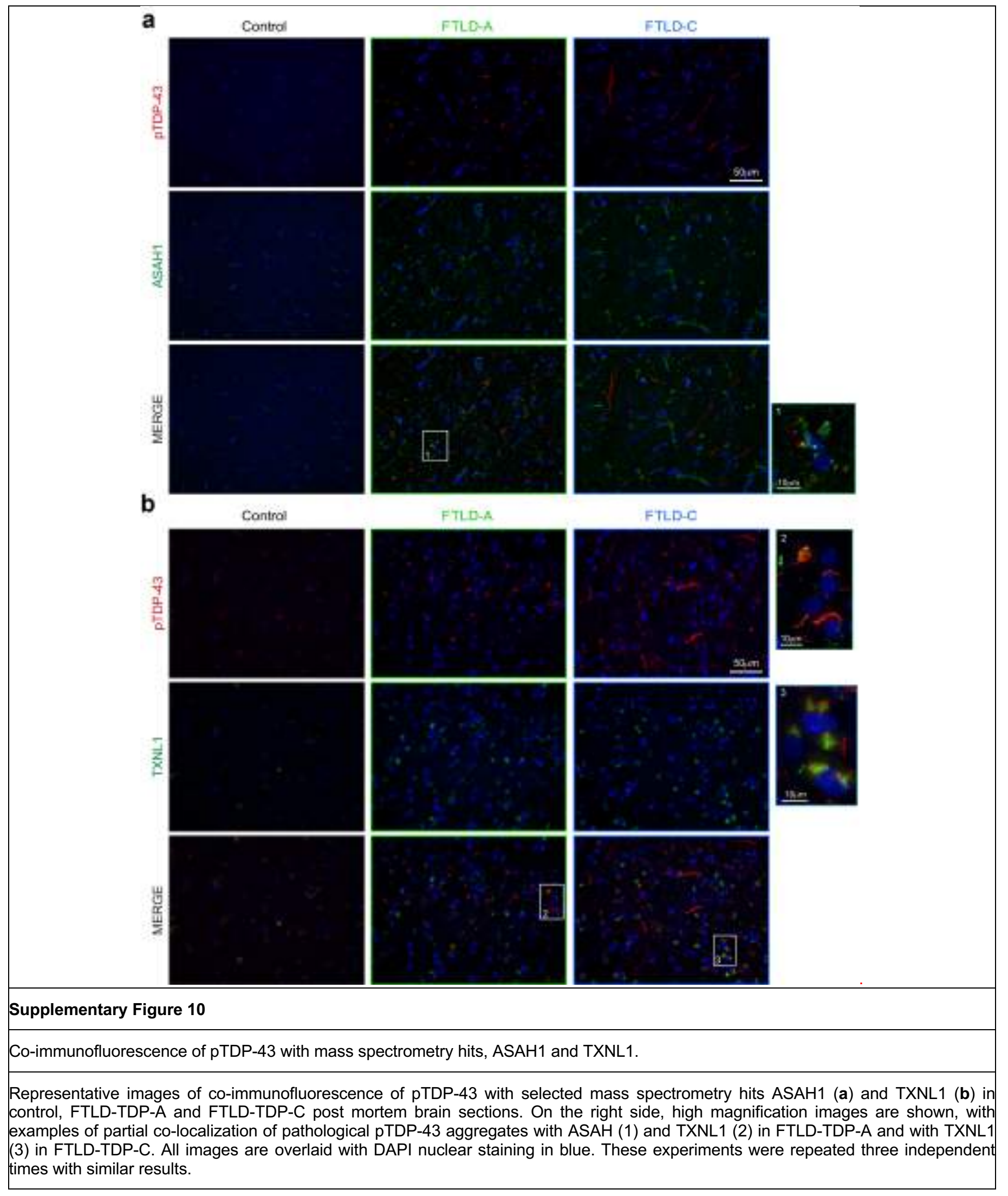


a

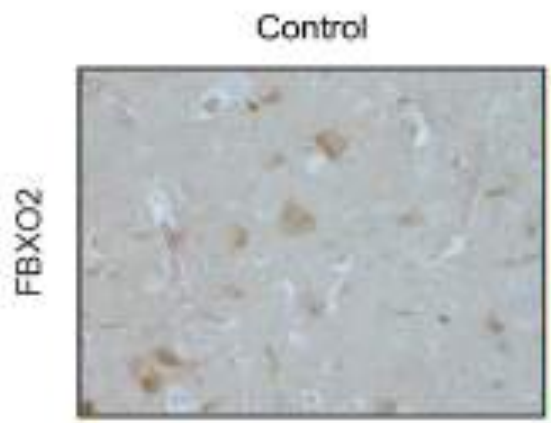

FTLD-A

FTLD-C
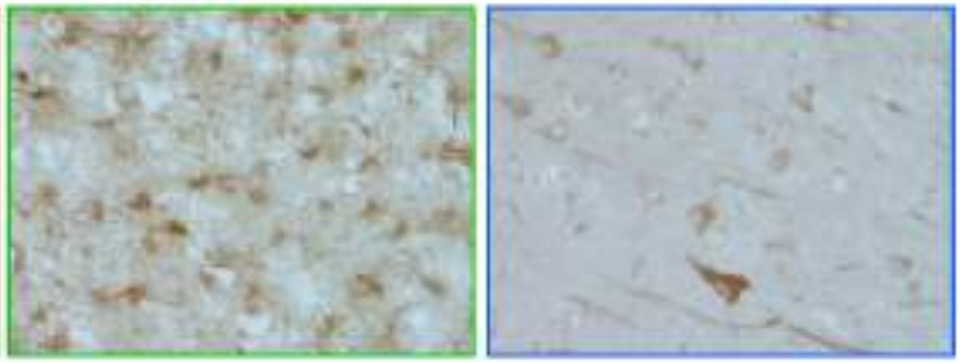

b

\section{Control}
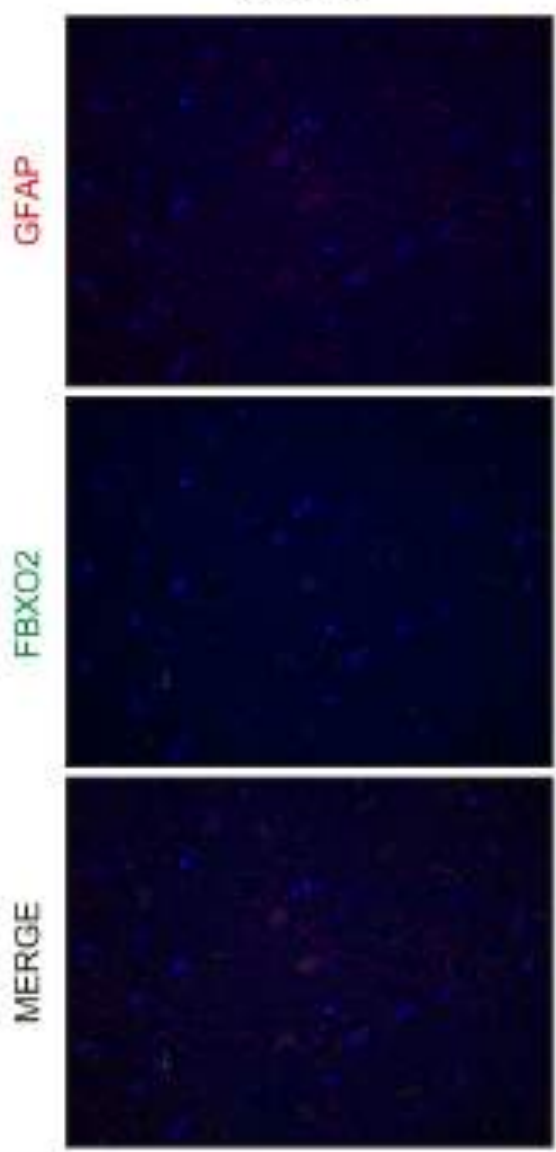

FTLD-A
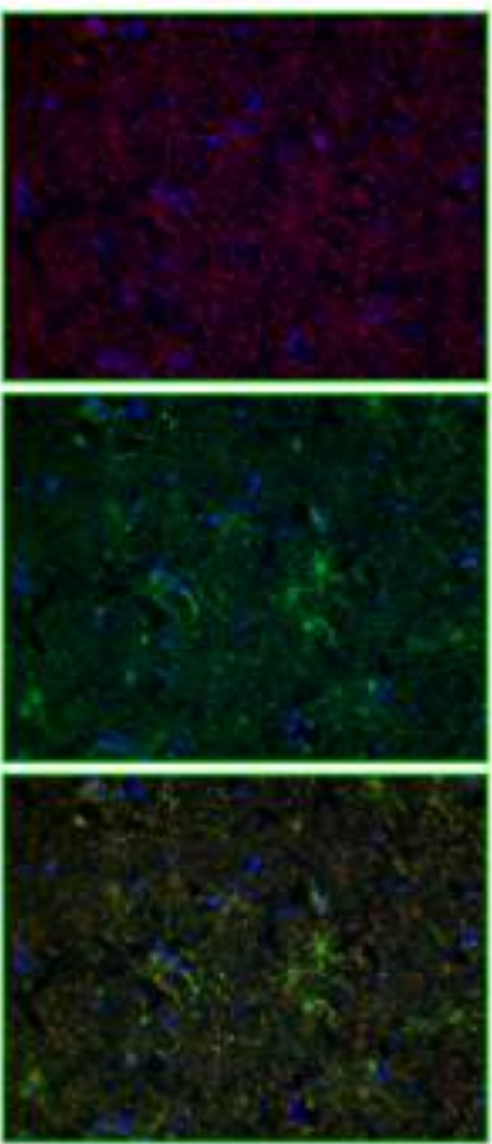

FTLD-C
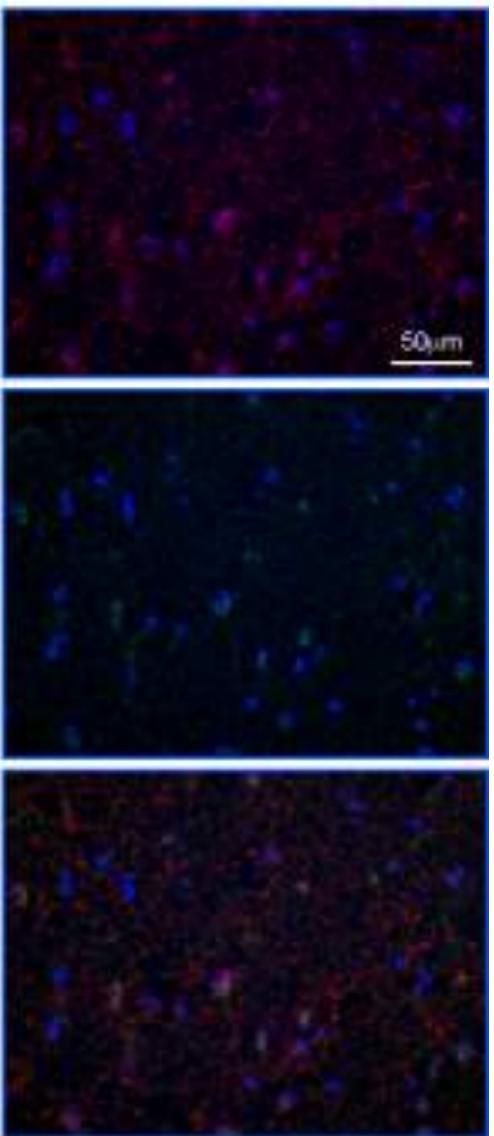

\section{Supplementary Figure 11}

FBXO2 upregulation in astrocytes in FTLD-TDP-A patients, but not FTLD-TDP-C.

a. Representative immunohistochemistry images showing FBXO2 immunoreactivity in control, FTLD-TDP-A and FTLD-TDP-C brain sections. b. Representative co-immunofluorescence of GFAP and FBXO2 in control, FTLD-TDP-A and FTLD-TDP-C brain sections corresponding to merged images in Fig. $\mathbf{2 h}$. All images are overlaid with DAPI nuclear staining in blue. These experiments were repeated three independent times with similar results. 


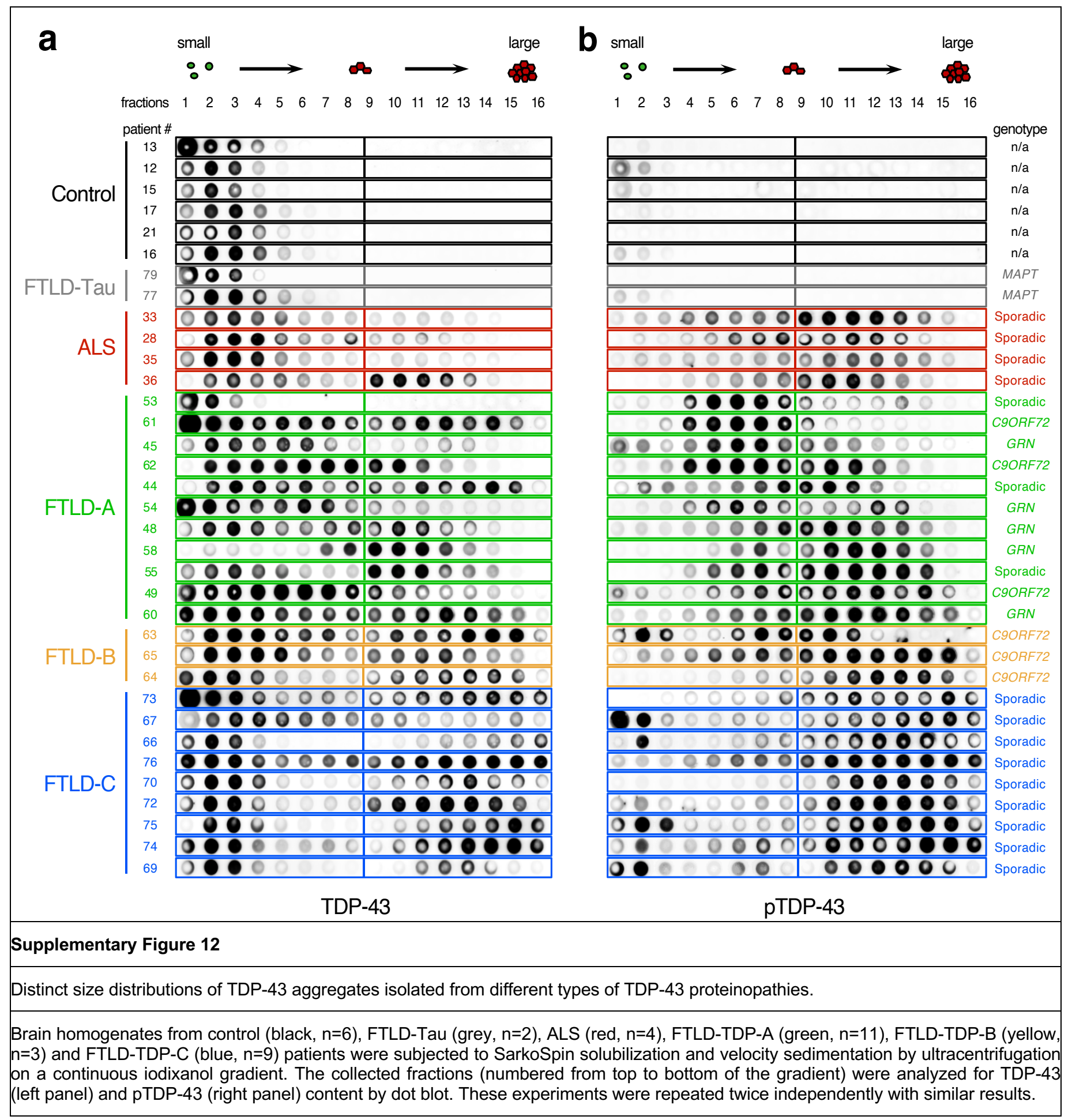




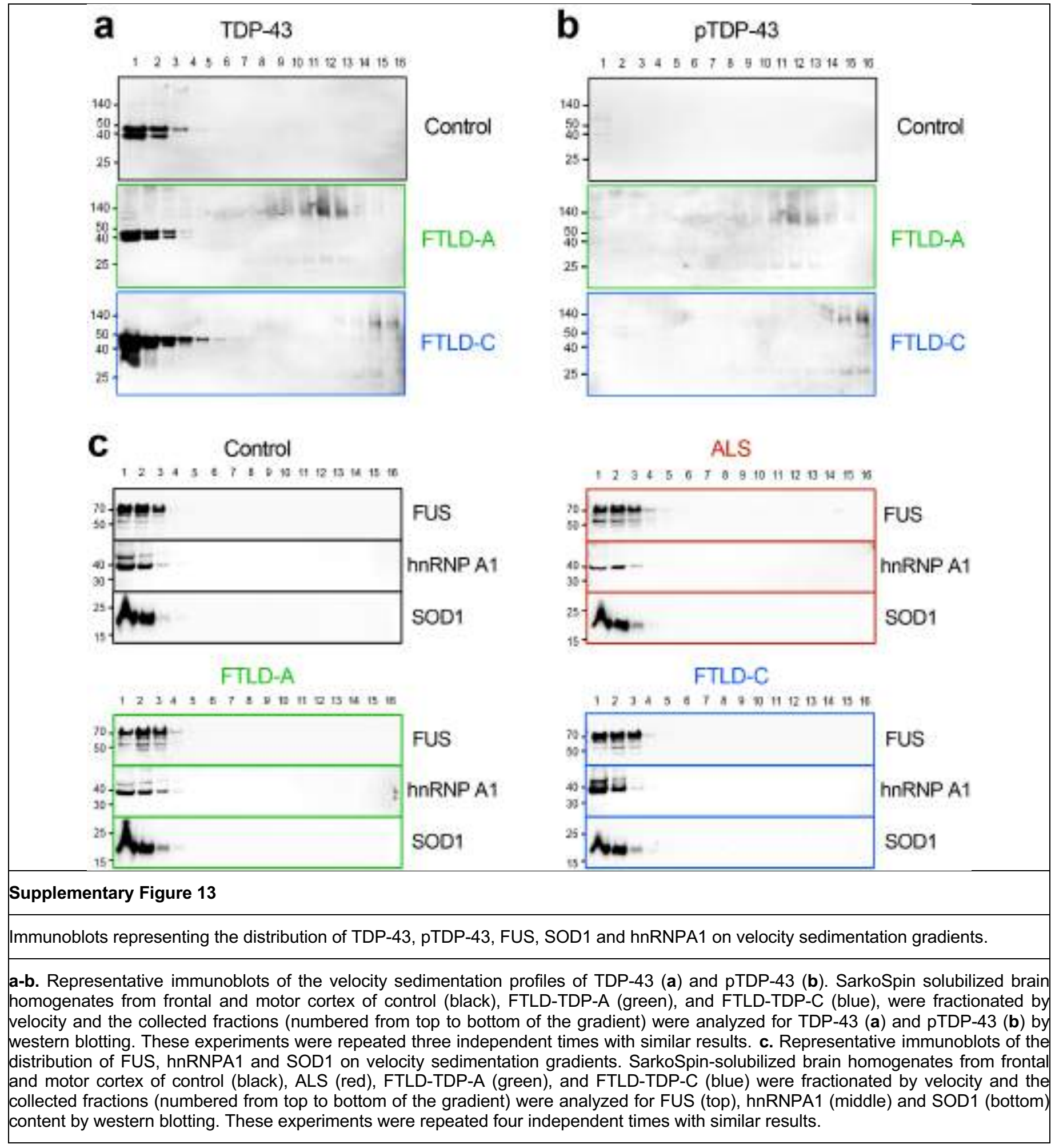




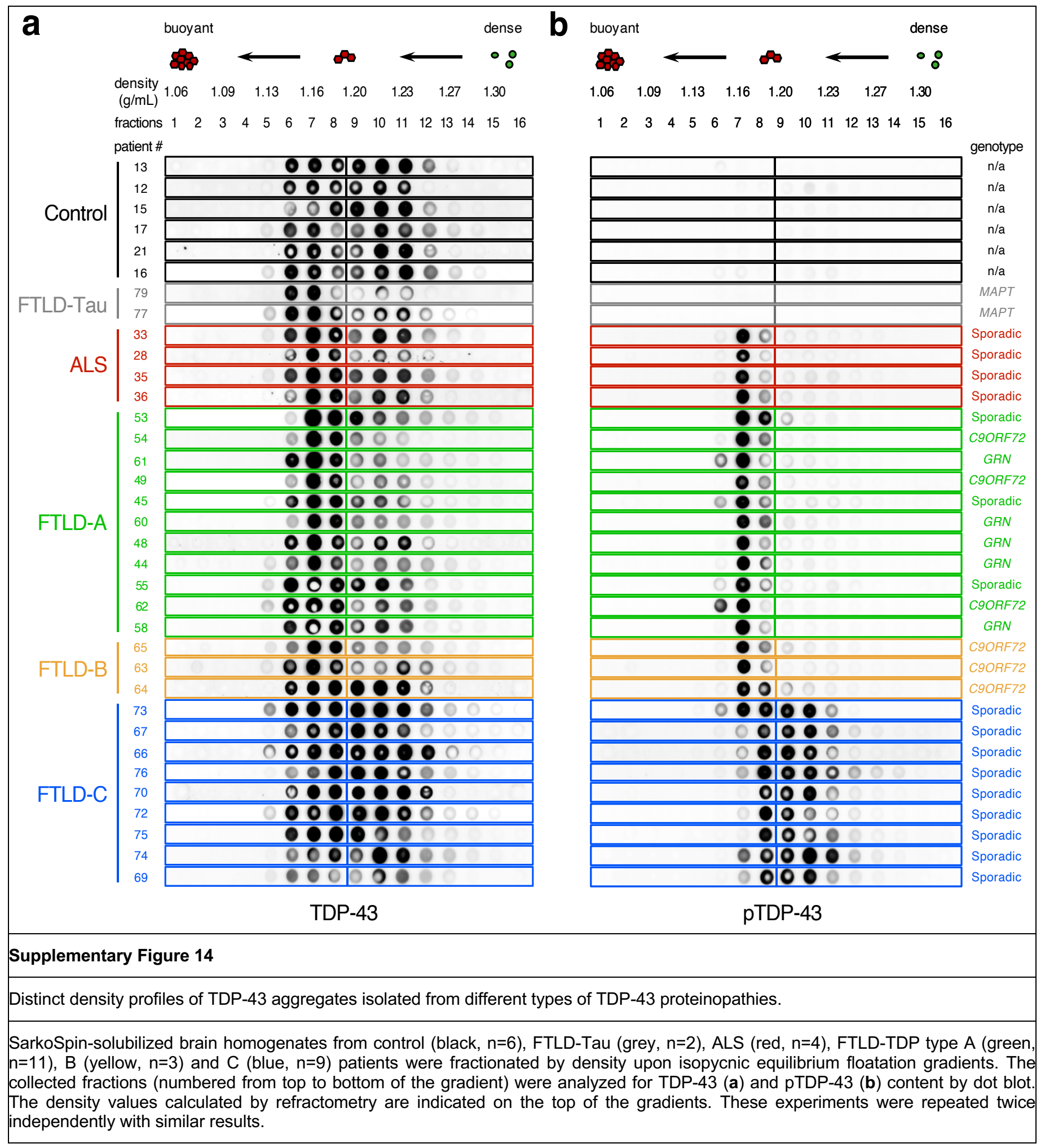




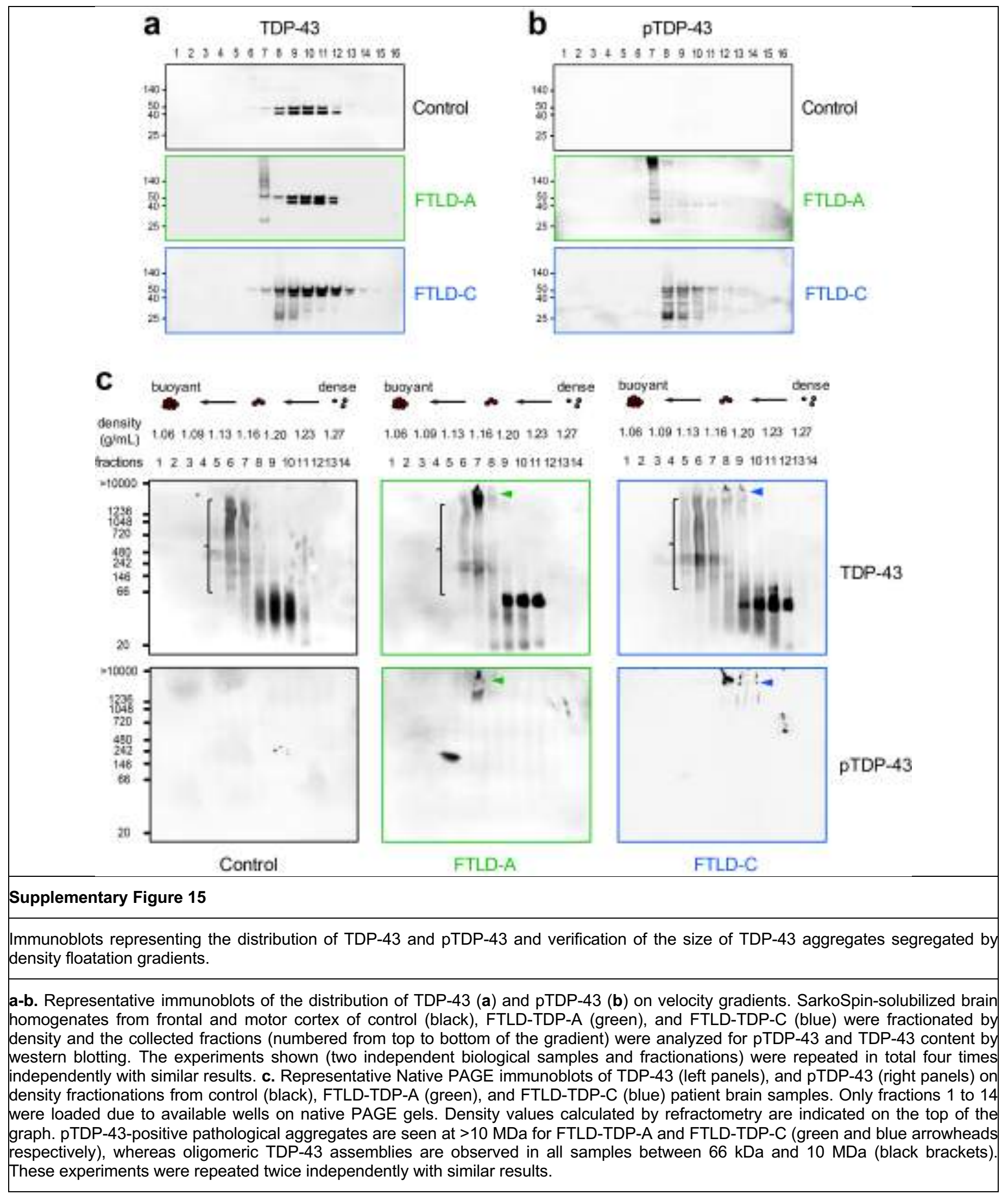



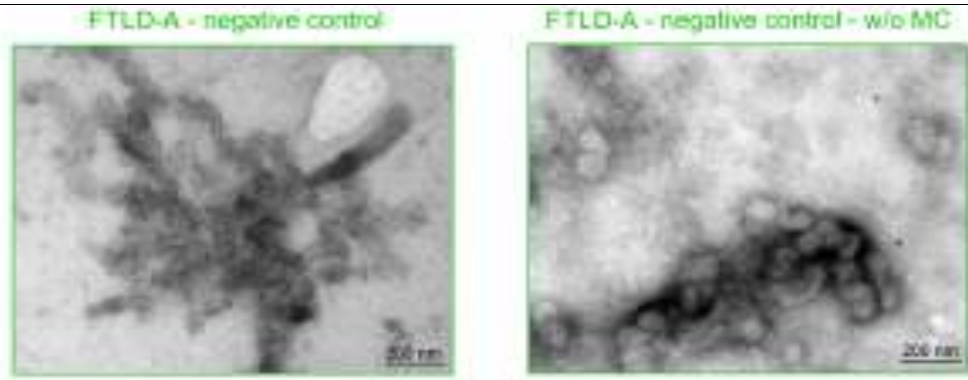

b

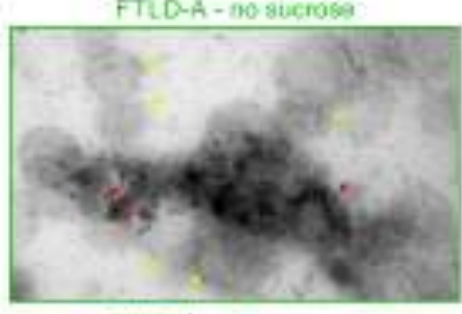

FTLD-A - who surroas

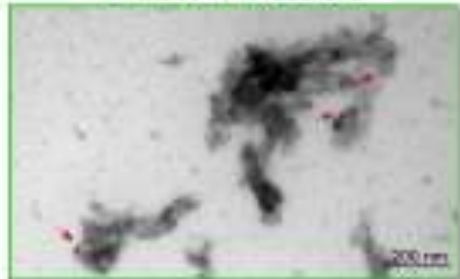

C
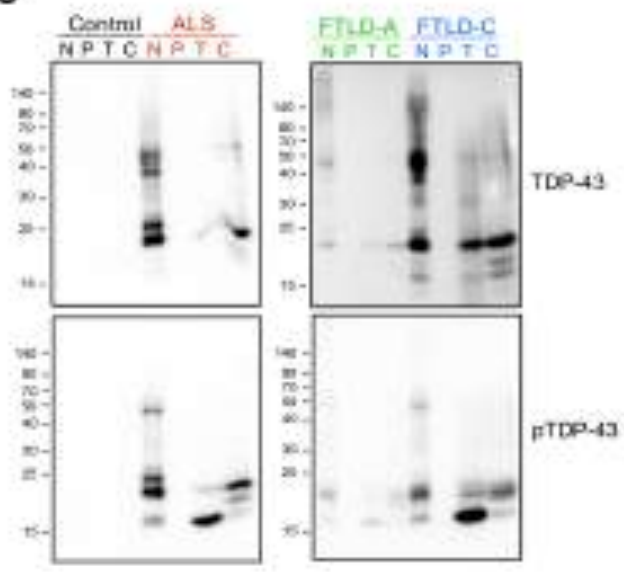

d

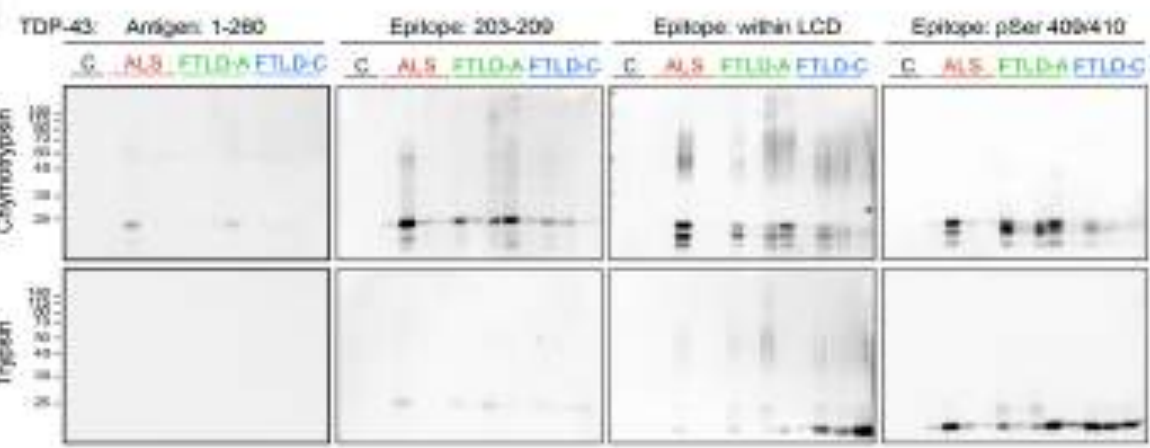

\section{Supplementary Figure 16}

Further structural analysis of pathological TDP-43 isolated from different disease subtypes.

a. TEM images of negative control, without the use of primary antibody, of immunolabeled FTLD-TDP-A insoluble material corresponding to TEM images from Fig. 5a-b, showing no unspecific binding of the gold-conjugated secondary antibody. Sample in panel on the left was prepared including the methylcellulose (MC) preservation step, and the sample represented on the right without the MC step. b. Upper panel: TEM image of immunolabeled FTLD-TDP-A aggregates (red arrows) with lipid contaminants (yellow arrows) surrounding the protein assemblies. Lower panel: TEM image of immunolabeled FTLD-TDP-A sample purified over sucrose cushion during SarkoSpin extraction, which efficiently removes the lipid contaminants (red arrows point to examples of gold-positive structures). $\mathbf{a}$. and b. experiments were independently repeated three times with similar results. c. Representative immunoblots of SarkoSpin pellets extracted from control, ALS, FTLD-TDP-A and FTLD-TDP-C brains after proteolytic digestion. Brain homogenates were digested by different proteases for 30 minutes at $37^{\circ} \mathrm{C}$ during SarkoSpin solubilization step. SarkoSpin pellets were subjected to SDS-PAGE and immunoblotted against total TDP-43 (top panels) and pTDP-43 (bottom panels). N - not treated; P - treated with 0.5 $\mathrm{mg} / \mathrm{ml}$ Proteinase K; T - treated with $0.1 \mathrm{mg} / \mathrm{ml}$ Trypsin; C - treated with $0.01 \mathrm{mg} / \mathrm{ml}$ Chymotrypsin. d. Full size representation of the immunoblots on Chymotrypsinized and Trypsinized SarkoSpin pellets shown in Fig. 5c. c. and d. experiments were repeated twice independently with similar results. 


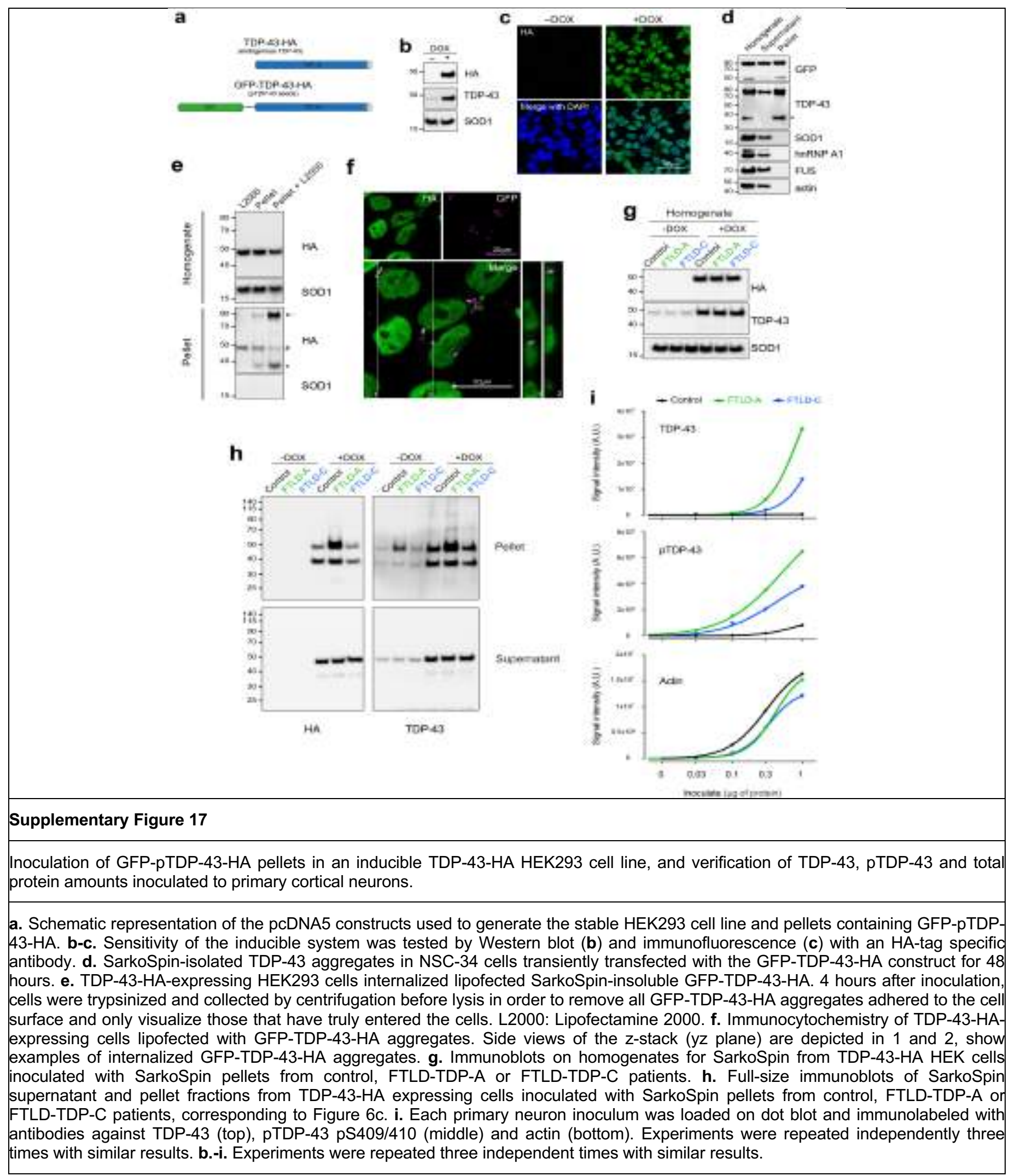

